\title{
Local Zeta Functions and Koba-Nielsen String Amplitudes
}

\author{
Miriam Bocardo-Gaspar ${ }^{1}$, Hugo García-Compeán ${ }^{2}$ (), Edgar Y. López ${ }^{2}$ and Wilson A. Zúñiga-Galindo ${ }^{3,4, *}$ \\ 1 Departamento de Matemáticas, Universidad de Guadalajara, Cucei, Blvd. Marcelino García Barragán \\ \#1421, 44430 Guadalajara, Mexico; miriam.bocardo@academicos.udg.mx \\ 2 Centro de Investigación y de Estudios Avanzados del I.P.N., Departamento de Física, Av. Instituto Politécnico \\ Nacional 2508, Col. San Pedro Zacatenco, 07360 Ciudad de México, Mexico; \\ compean@fis.cinvestav.mx (H.G.-C.); elopez@fis.cinvestav.mx (E.Y.L.) \\ 3 University of Texas Rio Grande Valley, School of Mathematical \& Statistical Sciences, \\ One West University Blvd, Brownsville, TX 78520, USA \\ 4 Centro de Investigación y de Estudios Avanzados del Instituto Politécnico Nacional Departamento de \\ Matemáticas, Unidad Querétaro Libramiento Norponiente \#2000, Fracc, Real de Juriquilla, \\ 76230 Santiago de Querétaro, Mexico \\ * Correspondence: wilson.zunigagalindo@utrgv.edu or wazuniga@math.cinvestav.edu.mx
}

Citation: Bocardo-Gaspar, M.; García-Compeán, H.; López, E.; Zúñiga-Galindo, W.A. Local Zeta Functions and Koba-Nielsen String Amplitudes. Symmetry 2021, 13, 967. https://doi.org/10.3390/ sym13060967

Academic Editor: Vladimir Al. Osipov

Received: 1 May 2021

Accepted: 24 May 2021

Published: 29 May 2021

Publisher's Note: MDPI stays neutral with regard to jurisdictional claims in published maps and institutional affiliations.

Copyright: (c) 2021 by the authors. Licensee MDPI, Basel, Switzerland. This article is an open access article distributed under the terms and conditions of the Creative Commons Attribution (CC BY) license (https:// creativecommons.org/licenses/by/ $4.0 /)$.

\begin{abstract}
This article is a survey of our recent work on the connections between Koba-Nielsen amplitudes and local zeta functions (in the sense of Gel'fand, Weil, Igusa, Sato, Bernstein, Denef, Loeser, etc.). Our research program is motivated by the fact that the $p$-adic strings seem to be related in some interesting ways with ordinary strings. $p$-Adic string amplitudes share desired characteristics with their Archimedean counterparts, such as crossing symmetry and invariance under Möbius transformations. A direct connection between $p$-adic amplitudes and the Archimedean ones is through the limit $p \rightarrow 1$. Gerasimov and Shatashvili studied the limit $p \rightarrow 1$ of the $p$-adic effective action introduced by Brekke, Freund, Olson and Witten. They showed that this limit gives rise to a boundary string field theory, which was previously proposed by Witten in the context of background independent string theory. Explicit computations in the cases of 4 and 5 points show that the Feynman amplitudes at the tree level of the Gerasimov-Shatashvili Lagrangian are related to the limit $p \rightarrow 1$ of the $p$-adic Koba-Nielsen amplitudes. At a mathematical level, this phenomenon is deeply connected with the topological zeta functions introduced by Denef and Loeser. A KobaNielsen amplitude is just a new type of local zeta function, which can be studied using embedded resolution of singularities. In this way, one shows the existence of a meromorphic continuations for the Koba-Nielsen amplitudes as functions of the kinematic parameters. The Koba-Nielsen local zeta functions are algebraic-geometric integrals that can be defined over arbitrary local fields (for instance $\mathbb{R}, \mathbb{C}, \mathbb{Q}_{p}, \mathbb{F}_{p}((T))$ ), and it is completely natural to expect connections between these objects. The limit $p$ tends to one of the Koba-Nielsen amplitudes give rise to new amplitudes which we have called Denef-Loeser amplitudes. Throughout the article, we have emphasized the explicit calculations in the cases of 4 and 5 points.
\end{abstract}

Keywords: string amplitudes; Koba-Nielsen amplitudes; Virasoro-Shapiro amplitudes; regularization; $p$-adic string amplitudes; local zeta functions; resolution of singularities

\section{Introduction}

In the recent years, the connections between string amplitudes and arithmetic geometry, $p$-adic analysis, combinatorics, etc. have been studied extensively, see e.g., [1-9] and the references therein.

The string amplitudes were introduced by Veneziano in the 60s, In [10], further generalizations were obtained by Virasoro [11], Koba and Nielsen [12], among others. In the 80s, Freund, Witten and Volovich, among others, studied string amplitudes at the tree level over different number fields, and suggested the existence of connections between these amplitudes, see e.g., $[4,13,14]$. In this framework the connection with local zeta 
functions appears naturally. This article is intended as a survey of our recent work on the connections between Koba-Nielsen amplitudes and local zeta functions [6-9] in the sense of Gel'fand, Weil, Igusa, Sato, Bernstein, Denef, Loeser, etc.

The $p$-adic string theories have been studied over the time with some periodic fluctuations in their interest (for some reviews, see [3-5,15]). Recently aconsiderable amount of work has been performed on this topic in the context of the AdS/CFT correspondence [16-19]. String theory with a $p$-adic worldsheet was proposed and studied for the first time in [20]. Later this theory was formally known as $p$-adic string theory. The adelic scattering amplitudes which are related to the Archimedean ones were studied in [21]. The tree-level string amplitudes were explicitly computed in the case of $p$-adic string worldsheet in [14,22]. One can obtain these amplitude, in a formal way, from a suitable action using general principles [23]. In [24], it was established that the tree-level string amplitudes may be obtained starting with a discrete field theory on a Bruhat-Tits tree. Determining the convergence of the amplitudes in momentum space is a difficult task, both in the standard and $p$-adic case; however, for the latter, this was precisely done for the $N$-point tree amplitudes in [8]. In this article we show (in a rigorous mathematical way) that the $p$-adic open string $N$-point tree amplitudes are bona fide integrals that admit meromorphic continuations as rational functions, this is done by associating to them multivariate local zeta functions (also called multivariate Igusa local zeta functions) [25-28]. In [6] we establish in a rigorous mathematical way that Koba-Nielsen amplitudes defined on any local field of characteristic zero (for instance $\mathbb{R}, \mathbb{C}, \mathbb{Q}_{p}$ ) are bona fide integrals that admit meromorphic continuations in the kinematic parameters. In the regularization process we use techniques of local zeta functions and embedded resolution of singularities.

The $p$-adic strings are related to ordinary strings at least in two different ways. First, connections through the adelic relations [21] and second, through the limit when $p \rightarrow 1[23,29]$. In [29] was showed that the limit $p \rightarrow 1$ of the effective action gives rise to a boundary string field theory, this was previously proposed by Witten in the context of background independent string theory [30,31]. The limit $p \rightarrow 1$ in the effective theory can be performed without any problem, since one can consider $p$ as a real parameter and take formally the limit $p \rightarrow 1$. The resulting theory is related to a field theory describing an open string tachyon [32]. In the limit $p \rightarrow 1$ also there are exact noncommutative solitons, some of these solutions were found in [33]. In [34] a very interesting physical interpretation of this limit was given in terms of a lattice discretization of ordinary string worldsheet. In the worldsheet theory we cannot forget the nature of $p$ as a prime number, thus the analysis of the limit is more subtle. The correct way of taking the limit $p \rightarrow 1$ involves the introduction of finite extensions of the $p$-adic field $\mathbb{Q}_{p}$. The totally ramified extensions give rise to a finer discretization of the worldsheet following the rules of the renormalization group [34].

In [35] Denef and Loeser showed that the limit $p$ approaches to one of a local zeta function give rise a new object called a topological zeta function. By using Denef-Loeser's theory of topological zeta functions, in [9], we show that the limit $p \rightarrow 1$ of tree-level $p$-adic string amplitudes give rise to new amplitudes, that we have named Denef-Loeser string amplitudes. Gerasimov and Shatashvili showed that in the limit $p \rightarrow 1$ the well-known non-local effective Lagrangian (reproducing the tree-level $p$-adic string amplitudes) gives rise to a simple Lagrangian with a logarithmic potential [29]. In [9], we conjecture that the Feynman amplitudes of this last Lagrangian are related to the Denef-Loser amplitudes.

In [7] we establish rigorously the regularization of the $p$-adic open string amplitudes, with Chan-Paton rules and a constant $B$-field. These amplitudes were originally introduced by Ghoshal and Kawano. To study these amplitudes, we introduce new multivariate local zeta functions depending on multiplicative characters (Chan-Paton factors) and a phase factor (the $B$-field) which involves an antisymmetric bilinear form. We show that these integrals admit meromorphic continuations in the kinematic parameters, this result allows us to regularize the Ghoshal-Kawano amplitudes, the regularized amplitudes do not have ultraviolet divergencies. The theory is only valid for $p$ congruent to $3 \bmod 4$, this is to preserve a certain symmetry. The limit $p \rightarrow 1$ is also considered for the noncommutative 
effective field theory and the Ghoshal-Kawano amplitudes. We show that in the case of four points, the limit $p \rightarrow 1$ of the regularized Ghoshal-Kawano amplitudes coincides with the Feynman amplitudes attached to the limit $p \rightarrow 1$ of the noncommutative GerasimovShatashvili Lagrangian.

We denote by $\mathbb{K}$ a local field of characteristic zero (for instance $\mathbb{R}, \mathbb{C}, \mathbb{Q}_{p}$ ), and set $f:=\left(f_{1}, \ldots, f_{m}\right)$ and $s:=\left(s_{1}, \ldots, s_{m}\right) \in \mathbb{C}^{m}$, where the $f_{i}(x)$ are non-constant polynomials in the variables $x:=\left(x_{1}, \ldots, x_{n}\right)$ with coefficients in $\mathbb{K}$. The multivariate local zeta function attached to $(f, \Theta)$, where $\Theta$ is a test function, is defined as

$$
Z_{\Theta}(f, s)=\int_{\mathbb{K}^{n}} \Theta(x) \prod_{i=1}^{m}\left|f_{i}(x)\right|_{\mathbb{K}_{i=1}^{s}}^{s_{i}} \prod_{i}^{n} d x_{i}, \quad \text { when } \operatorname{Re}\left(s_{i}\right)>0 \text { for all } i,
$$

and where $\prod_{i=1}^{n} d x_{i}$ is the normalized Haar measure of $\left(\mathbb{K}^{n},+\right)$. These integrals admit meromorphic continuations to the whole $\mathbb{C}^{m},[25,26,36]$, see also [37,38]. The study of Archimedean and non-Archimedean local zeta functions was started by Weil in the 60 $\mathrm{s}$, in connection to the Poisson-Siegel formula. In the $70 \mathrm{~s}$, Igusa developed a uniform theory for local zeta functions in characteristic zero $[25,26]$, see also $[36,39,40]$. In the $p$-adic setting, the local zeta functions are connected with the number of solutions of polynomial congruences mod $p^{l}$ and with exponential sums $\bmod p^{l}$ [28]. Recently, Denef and Loeser introduced the motivic zeta functions which constitute a vast generalization of $p$-adic local zeta functions [41,42].

In the $50 \mathrm{~s}$, Gel'fand and Shilov studied the local zeta functions attached to real polynomials in connection with fundamental solutions, indeed, the meromorphic continuation of Archimedean local zeta functions implies the existence of fundamental solutions (i.e., Green functions) for differential operators with constant coefficients. This fact was established, independently, by Atiyah [43] and Bernstein [44]. It is relevant to mention here that in the $p$-adic framework, the existence of fundamental solutions for pseudodifferential operators is also a consequence of the fact that the Igusa local zeta functions admit a meromorphic continuation, see ([45], Chapter 5), ([46], Chapter 10). This analogy turns out to be very important in the rigorous construction of quantum scalar fields in the $p$-adic setting, see [47] and the references therein.

Take $N \geq 4$, and complex variables $s_{1 j}$ and $s_{(N-1) j}$ for $2 \leq j \leq N-2$ and $s_{i j}$ for $2 \leq i<j \leq N-2$. Put $s:=\left(s_{i j}\right) \in \mathbb{C}^{D}$, where $D=\frac{N(N-3)}{2}$ denotes the total number of indices $i j$. In [6] we introduce the multivariate local zeta function

$$
Z_{\mathbb{K}}^{(N)}(s):=\int_{\mathbb{K}^{N-3}} \prod_{i=2}^{N-2}\left|x_{j}\right|_{\mathbb{K}}^{s_{1 j}}\left|1-x_{j}\right|_{\mathbb{K}}^{s_{(N-1) j}} \prod_{2 \leq i<j \leq N-2}\left|x_{i}-x_{j}\right|_{\mathbb{K}}^{s_{i j}} \prod_{i=2}^{N-2} d x_{i},
$$

where $\prod_{i=2}^{N-2} d x_{i}$ is the normalized Haar measure on $\mathbb{K}^{N-3}$. We called these integrals KobaNielsen local zeta functions. In ([6], Theorems 4.1 and 6.1), we show that these functions are bona fide integrals, which are holomorphic in an open part of $\mathbb{C}^{D}$, containing the set given by

$$
\frac{-2}{N-2}<\operatorname{Re}\left(s_{i j}\right)<\frac{-2}{N} \text { for all } i j .
$$

Furthermore, they admit meromorphic continuations to the whole $\mathbb{C}^{D}$.

The Koba-Nielsen open string amplitudes for $N$-points over $\mathbb{K}$ are formally defined as

$$
A_{\mathbb{K}}^{(N)}(\boldsymbol{k}):=\int_{\mathbb{K}^{N-3}} \prod_{i=2}^{N-2}\left|x_{j}\right|_{\mathbb{K}}^{k_{1} k_{j}}\left|1-x_{j}\right|_{\mathbb{K}}^{k_{N-1} k_{j}} \prod_{2 \leq i<j \leq N-2}\left|x_{i}-x_{j}\right|_{\mathbb{K}}^{k_{i} k_{j}} \prod_{i=2}^{N-2} d x_{i},
$$

where $\boldsymbol{k}=\left(\boldsymbol{k}_{1}, \ldots, \boldsymbol{k}_{N}\right), \boldsymbol{k}_{i}=\left(k_{0, i}, \ldots, k_{l, i}\right) \in \mathbb{R}^{l+1}$, for $i=1, \ldots, N(N \geq 4)$, is the momentum vector of the $i$-th tachyon (with Minkowski product $\boldsymbol{k}_{i} \boldsymbol{k}_{j}=-k_{0, i} k_{0, j}+k_{1, i} k_{1, j}+\cdots+$ $\left.k_{l, i} k_{l, j}\right)$, obeying 


$$
\sum_{i=1}^{N} \boldsymbol{k}_{i}=\mathbf{0}, \quad \boldsymbol{k}_{i} \boldsymbol{k}_{i}=2 \text { for } i=1, \ldots, N .
$$

The parameter $l$ is an arbitrary positive integer. Typically, $l$ is taken to be 25 for bosonic strings. However, we do not require using the critical dimension. We choose units such that the tachyon mass is $m^{2}=-2$.

In the real case, $A_{\mathbb{R}}^{(N)}(\boldsymbol{k})$ is (up to multiplication by a positive constant) the open Koba-Nielsen amplitude of $N$-points, see ([4], Section 8), ([48], Section 2). If $N=4, A_{\mathbb{R}}^{(4)}(\boldsymbol{k})$ is the Veneziano amplitude [10]. In the complex case, by using the results of ([48], Section 2), in [49], the $N$-point, closed string amplitude at the tree level is the product of $A_{\mathbb{C}}^{(N)}(\boldsymbol{k})$ times a polynomial function in the momenta $k$. This fact implies that the techniques and results presented in [6] are applicable to classical closed string amplitudes at the tree level.

We use the integrals $Z_{\mathbb{K}}^{(N)}(s)$ as regularizations of the amplitudes $A_{\mathbb{K}}^{(N)}(\boldsymbol{k})$. More precisely, we redefine

$$
A_{\mathbb{K}}^{(N)}(\boldsymbol{k})=\left.Z_{\mathbb{K}}^{(N)}(\boldsymbol{s})\right|_{s_{i j}=\boldsymbol{k}_{i} \boldsymbol{k}_{j}}
$$

where $Z_{\mathbb{K}}^{(N)}(s)$ now denotes the meromorphic continuation of (1) to the whole $\mathbb{C}^{D}$, see ([6], Theorem 6.1). Furthermore, $A_{\mathbb{K}}^{(N)}(\boldsymbol{k})$ extends to a meromorphic function to the whole $\mathbb{C}^{N(l+1)}$, see ([6], Theorem 7.1).

The articles $[6,8]$ deal with the meromorphic continuation of local zeta functions of type $Z_{\mathbb{K}}^{(N)}(s)$, but their results are complementary. The main result of [8] is the existence of a meromorphic continuation for $Z_{\mathbb{K}}^{(N)}(s)$ in the case in which $\mathbb{K}$ is a non-Archimedean local field of arbitrary characteristic. For instance $\mathbb{Q}_{p}$ or $\mathbb{F}_{q}((T))$, the field of formal Laurent series with coefficients in a finite field $\mathbb{F}_{q}$. The methods used are based on the stationary phase formula, which allows the explicit calculation of $Z_{\mathbb{K}}^{(N)}(s)$. On the other hand, the results presented in [6] depend completely on Hironaka's resolution of singularities theorem [50], which is available only for fields of characteristic zero. In this framework, the results of [6] show that for any field of characteristic zero $\mathbb{K}$, all the zeta functions $Z_{\mathbb{K}}^{(N)}(s)$ converge on a common domain, see (2), and that the possible poles can be described in a geometric form. We propose the following conjecture:

Conjecture 1. There is a meromorphic function $\Gamma(s)$ in $\mathbb{C}^{D}$ such that

$$
Z_{\mathbb{R}}^{(N)}(s)=\Gamma(s) \prod_{p<\infty} Z_{\mathbb{Q}_{p}}^{(N)}(s) \text { for any } s \in \mathbb{C}^{D}
$$

This conjecture and ([6], Theorem 6.1) imply that $\prod_{p<\infty} Z_{\mathbb{Q}_{p}}^{(N)}(s)$ admits a meromorphic continuation to the whole $\mathbb{C}^{D}$. By using (3), this conjecture implies that

$$
A_{\mathbb{R}}^{(N)}(s)=\Gamma(s) \prod_{p<\infty} A_{\mathbb{Q}_{p}}^{(N)}(s)
$$

is meromorphic function in $\mathbb{C}^{D}$. Freund and Witten established (formally) that the ordinary Veneziano and Virasoro-Shapiro four-particle scattering amplitudes can be factored in terms of an infinite product of non-Archimedean string amplitudes [21]. More precisely, they established that the product of the $p$-adic string amplitudes multiplied by the crossingsymmetric Veneziano amplitude is equal to one. Later Aref'eva, Dragovich and Volovich noted that the infinite product used by Freund and Witten is divergent, and established that the product of the $p$-adic string amplitudes multiplied by the crossing-symmetric Veneziano amplitude is equal to an expression containing the Riemann zeta function [51]. 
We already mentioned that the Denef-Loeser amplitudes $A_{\text {top }}^{(N)}(s)$ are the limit of the Koba-Nielsen amplitudes $A_{\mathbb{Q}_{p}}^{(N)}(s)$ when $p$ tends to one. This is a consequence of the fact that $Z_{\text {top }}^{(N)}(s)=\lim _{p \rightarrow 1} Z_{\mathbb{Q}_{p}}^{(N)}(s)$, which in turn is a consequence of the motivic integration theory, see e.g., [41,52]. Motivic integration is a generalization of the $p$-adic integration and the integration with respect to the Euler characteristic. By using this theory one can construct a function $Z_{\mathbb{C}((T))}^{(N)}(\boldsymbol{L})$ such that

$$
Z_{\mathbb{Q}_{p}}^{(N)}(s)=\left.Z_{\mathbb{C}((T))}^{(N)}(\boldsymbol{L})\right|_{\boldsymbol{L}=p} \text { and } Z_{\text {top }}^{(N)}(\boldsymbol{s})=\left.Z_{\mathbb{C}((T))}^{(N)}(\boldsymbol{L})\right|_{\boldsymbol{L}=1},
$$

which in turn implies the existence of new type of 'motivic string amplitude' at the three level $A_{\mathbb{C}((T))}^{(N)}(\boldsymbol{L})$ which is a generalization of the $p$-adic Koba-Nielsen and Denef-Loeser amplitudes.

Problem 1. Does the motivic string amplitudes $A_{\mathbb{C}((T))}^{(N)}(\boldsymbol{L})$ have a physical meaning? Is there an effective action such that the Feynman integrals at the three level are exactly the $A_{\mathbb{C}((T))}^{(N)}(\boldsymbol{L})$ ?

In [9], we verified that the four and five-point Feynman amplitudes of the Gerasimov-Shatashvili Lagrangian are related by $\lim _{p \rightarrow 1} A_{\mathbb{Q}_{p}}^{(N)}(s)$. Then we propose the following conjecture:

Conjecture 2. The Feynman amplitudes at the three level of the Gerasimov-Shatashvili Lagrangian are by related $\lim _{p \rightarrow 1} A_{\mathbb{Q}_{p}}^{(N)}(s)$.

A precise formulation of this conjecture is given at the end of Section 6.

This survey is organized as follows. In Section 2, we review briefly basic aspects of string amplitudes. Section 3 aims to provide an introduction to $p$-adic analysis. Section 4 is dedicated to the $p$-adic Koba-Nielsen amplitudes. We compute explicitly the amplitudes for 4 and 5 points. At the end of the section we provide an outline of the proof that the $p$-adic Koba-Nielsen amplitudes admit meromorphic continuations as rational functions in $p^{-k_{i} k_{j}}$. The Koba-Nielsen amplitudes can be defined over any local field (i.e., $\mathbb{R}, \mathbb{C}$, $\mathbb{Q}_{p}, \mathbb{F} p((T))$, etc.). Section 5 is dedicated to amplitudes defined on local fields such as $\mathbb{Q}_{p}, \mathbb{F} p((T))$. A central idea is that the limit $p$ tends to one of the $p$-adic Koba-Nielsen amplitudes gives a new amplitude, the Denef-Loeser amplitude. We compute the DenefLoeser amplitudes for 4 and 5 points. Section 6 deals with the physical interpretation of the limit $p \rightarrow 1$ of $p$-adic string amplitudes. In this section we show, in the case of 4 and 5 particles, that the Feynman integrals at the tree level of the Gerasimov-Shatashvili Lagrangian are related Denef-Loeser amplitudes. We also give a precise formulation of Conjecture 2. Section 7 deals with the $N$-point $p$-adic string amplitudes, with Chan-Paton rules and a constant $B$-field. We emphasize the explicit calculations of these amplitudes in the cases $N=4,5$. In Section 8 , we briefly review some basic definitions and some central results about analytic manifolds on local fields of characteristic zero, Hironaka's resolution of singularities theorem and multivariate Igusa zeta functions. In Section 10, we give an outline of the proof of the fact that Koba-Nielsen amplitudes defined on local fields of characteristic zero admit meromorphic continuations in $\boldsymbol{k}_{i} \boldsymbol{k}_{j} \in \mathbb{C}$. In the cases $N=4,5$, we show explicitly the existence of a meromorphic continuations for amplitudes by outlining the construction of certain embedded resolution of singularities.

\section{Generalities of String Amplitudes}

String theory is the theory of tiny one-dimensional extended objects propagating in an underlying Riemannian or pseudo-Riemannian spacetime manifold $M$. The theory is defined as a theory for a dynamical embedding map $X: \Sigma_{g, b, N} \rightarrow M$, where $\Sigma_{g, b, N}$ is a compact and oriented Riemann surface with a possible nonempty boundary $\left(\partial \sum \neq 0\right)$ 
characterized by the genus $g$, their number of boundaries $b$ and $N$ marked points. $\Sigma_{g, b, N}$ has local coordinates $\sigma=\left(\sigma^{0}, \sigma^{1}\right)\left(\sigma^{a}\right.$ with $\left.a=0,1\right)$. Under a Wick rotation $\sigma^{1} \rightarrow \sigma^{1}$ and $\sigma^{2}=i \sigma^{0}$, the metric written in terms of coordinates $\left(\sigma^{1}, \sigma^{2}\right)$ becomes a metric with Euclidean signature. In addition to $X$ there is a non-dynamical filed $h_{a b}$ on $\Sigma_{g, N}$, which is the intrinsic metric. The string may oscillate in the target space $M$ and it has an infinite number of quantum modes of oscillation carrying a representation of the Poincaré group of the target space ( In the present review will be enough to consider $M$ to be the flat Minkowskian spacetime) and characterized by a mass and a spin. In the bosonic string the spectrum consists of a tachyonic mode with negative square mass. There are a finite number of massless modes described by massless fields on $M$. These modes are the target space metric $g_{\mu \nu}$ with Lorentzian signature $(-,+, \ldots,+)$, the antisymmetric field $B_{\mu \nu}$, and the dilaton field $\Phi$, where $\mu, v=0, \ldots, D-1 . M$ is the target space which is a Riemannian manifold of 26 dimensions in the case of the bosonic string and 10 dimensions for the superstring [53-55]. The Euclidean worldsheet action for the bosonic open string is given by

$$
I=S+S^{\prime}
$$

where

$$
\begin{aligned}
S & =\frac{T}{2} \int_{\Sigma} d^{2} \sigma \sqrt{h}\left(h^{a b} \partial_{a} X^{\mu} \partial_{b} X^{v} g_{\mu \nu}(X)-2 \pi i \alpha^{\prime} B_{\mu \nu} \epsilon^{a b} \partial_{a} X^{\mu} \partial_{b} X_{v}\right) \\
& =\frac{T}{2} \int_{\Sigma} d^{2} \sigma \sqrt{h} h^{a b} \partial_{a} X^{\mu} \partial_{b} X^{v} g_{\mu \nu}(X)-\frac{i}{2} \int_{\partial \Sigma} d l B_{\mu \nu} X^{\mu} \partial_{t} X^{v},
\end{aligned}
$$

where $T=\frac{1}{2 \pi \alpha^{\prime}}$ is the string tension, here $\alpha^{\prime}=\ell_{S}^{2}$ and $\ell_{S}$ stands for the string length. In the above action the physical degrees of freedom are $X^{\mu}$ and $h_{a b}, \partial_{t}$ is the tangential derivative along $\partial \Sigma$ and $B_{\mu \nu}$ is an antisymmetric field. Moreover, the action $S^{\prime}$ is given by

$$
S^{\prime}=\Phi_{0} \chi(\Sigma)
$$

where $\chi(\Sigma)=\frac{1}{4 \pi} \int d^{2} \sigma \sqrt{h} R+B T$ is the Euler characteristic of $\Sigma$ and BT is the GibbonsHawking boundary term that we encode in $\chi(\Sigma)$ with the number of boundaries $b$. Notice that the vacuum expectation value of the dilaton $\Phi_{0}$ can also be incorporated in the action through this topological term. It is also worth noting that this is the most general action that has worldsheet reparametrization invariance, in particular Weyl invariance $\left(h_{a b} \rightarrow \Lambda(\sigma) h_{a b}\right)$, and is renormalizable by power counting (this implies that there must be two worldsheet derivatives in each term). The second term is analogous to placing an electromagnetic field in the background with which the string interacts; it is a gauge field in that sense. Mathematically it is the integration of the pullback of the $B$-field to the string worldsheet. If we define $A_{\mu}:=B_{\mu \nu} X^{\nu}$, the second term of the second line in (5) may be written as

$$
\frac{i}{2} \int_{\partial \Sigma} A_{\mu} \partial_{t} X^{\mu}
$$

which is exactly the term considered in [56] where the partition function of the action was obtained explicitly at tree level. Strings propagating in non-trivial backgrounds have been considered in other works [57].

We now consider flat space as well as a constant $B$-field. The open string action (5) determines the Neumann boundary conditions

$$
g_{\mu \nu} \partial_{\sigma} X^{v}+\left.2 \pi i \alpha^{\prime} B_{\mu \nu} \partial_{t} X^{v}\right|_{\partial \Sigma}=0,
$$

where $\partial_{\sigma}$ is the normal derivative to $\partial \Sigma$. We should note that these conditions are in general complex, because we have taken a Wick rotation to a Euclidean signature. We will be concerned about the case when the string worldsheet $\Sigma$ is a disk, which corresponds to the open string in the classical limit. It is known that the disk can be transformed into the 
upper half plane via a conformal transformation, whose boundary is the real line. In these variables the Neumann boundary conditions are [58]

$$
g_{\mu \nu}(\partial-\bar{\partial}) X^{v}+\left.2 \pi \alpha^{\prime} B_{\mu \nu}(\partial+\bar{\partial}) X^{v}\right|_{z=\bar{z}}=0,
$$

where $\partial=\partial / \partial z$ and $\bar{\partial}=\partial / \partial \bar{z}$, with $\operatorname{Im} z \geq 0$.

The perturbative scattering amplitudes of $N$ particles are defined as the correlation function of $N$ vertex operators $\mathcal{V}_{\Lambda_{i}}\left(\boldsymbol{k}_{i}\right)$. Furthermore, these amplitudes admit an expansion over the genus $g$ of $\chi(\Sigma)=2-2 g-b$ as follows:

$$
\mathcal{A}\left(\Lambda_{1}, \boldsymbol{k}_{1}, \ldots, \Lambda_{N}, \boldsymbol{k}_{N}\right)=\sum_{g=0}^{\infty} \lambda_{s}^{2 g-2+b} A_{g}\left(\Lambda_{1}, \boldsymbol{k}_{1}, \ldots, \Lambda_{N}, \boldsymbol{k}_{N}\right),
$$

where $\lambda_{s}:=e^{\Phi_{0}}$ is the coupling constant of string and $A_{g}$ is the Feynman functional integral

$$
A_{g}\left(\Lambda_{1}, \boldsymbol{k}_{1}, \ldots, \Lambda_{N}, \boldsymbol{k}_{N}\right)=\frac{1}{\operatorname{Vol}} \int_{\mathfrak{M}_{g, b, N}} \operatorname{DXDh} \exp (-S) \prod_{i=1}^{N} \mathcal{V}_{\Lambda_{i}}\left(\boldsymbol{k}_{i}\right),
$$

here Vol stands for the volume of the symmetry groups leaving invariant the action (4). Here the integration is carried out over $\mathfrak{M}_{g, b, N}$ the moduli space of Riemann surfaces of genus $g, b$ boundaries and $N$ marked points inserted on the boundary. The difficulty of integration increases with higher values of $g, b$ and $N$. Fortunately it can be carried out in an explicit form for a few simple cases with lower values of $g, b$ and $N$. Thus, for instance, the case for closed strings with genus $g=0$ and $N=4$, or open strings with $g=0$, $b=1$ and $N=4$, are explicitly performed. Vertex operators $\mathcal{V}_{\Lambda_{i}}\left(\boldsymbol{k}_{i}\right)$ are functionals of the embedding fields $X$ and their derivatives. They are given by

$$
\mathcal{V}_{\Lambda}(\boldsymbol{k})=\int d^{2} \sigma \sqrt{h} W_{\Lambda}(\sigma) e^{i k X(\sigma)},
$$

where $W_{\Lambda}(\sigma)$ represents a functional of $X$ and its derivatives associated with the species of field in the string spectrum, $X(\sigma)=\left(X^{0}, \ldots, X^{25}\right), k=\left(k^{0}, \ldots, k^{25}\right)$ are the position and momentum vectors in target space $M$ and

$$
k X(\sigma):=\sum_{\mu, v=0}^{25} k^{\mu} \eta_{\mu \nu} X^{v}(\sigma)
$$

is the Minkowskian inner product. For instance for tachyons $\Lambda=t, W_{t}(\sigma)=1$. For gauge fields $\Lambda=A$ and $W_{A}(\sigma)=\varepsilon_{\mu} \partial_{t} X^{\mu}$, where $\varepsilon_{\mu}$ is the polarization vector. The graviton is $W_{G}(\sigma)=\varepsilon_{\mu v} h^{a b} \partial_{a} X^{\mu} \partial_{b} X^{v}$, where $\varepsilon_{\mu \nu}$ is the polarization tensor. For bosonic closed strings, the tachyon is the lowest state and it has a $k^{2}=\frac{4}{\alpha^{\prime}}$, where $k^{2}:=k \boldsymbol{k}$ is the Minkowskian norm. The open string tachyon has $k^{2}=\frac{1}{\alpha^{\prime}}$. In units where the Regge slope $\alpha^{\prime}=\frac{1}{2}$ or where the string tension $T=\frac{1}{\pi}$ we have that for the closed string tachyon $k^{2}=8$ and for the open string $k^{2}=2$, which will be used in the following sections of the present survey. The massless open string gauge field and the closed string graviton have $k^{2}=0$.

For open strings one can see that in the functional integral with action (5) the expansion in Taylor's series of exponential of the second term of (5), leads to a superposition of amplitudes with different powers of the vertex operator of the gauge field (6) inserted in the boundary $\partial \Sigma$.

From now on, we restrict ourselves to the case of bosonic closed strings with $g=0$ and bosonic open strings with $g=0$ and $b=1$, i.e., the 2 -sphere and the disk. For closed strings, by worldsheet diffeomorphisms and Weyl transformations of $\Sigma$, allow us to fix the worldsheet metric $h_{a b}$ to the Euclidean flat metric $\delta_{a b}$. This is the called the conformal gauge. In the absence of a $B$-field, there is a remnant $S L(2, \mathbb{C})$ symmetry which cannot be fixed by the local Weyl symmetry. This is called the conformal Killing symmetry. This symmetry does act on the complex worldsheet coordinates $(z, \bar{z})$ of the 2-sphere separately. In the holomorphic sector (for the anti-holomorphic sector a similar argument is applied) 


$$
z \mapsto \frac{a z+b}{c z+d}
$$

where $a d-b c=1$ and $a, b, c, d \in \mathbb{R}$. This symmetry allows the choice of three points on the sphere which could be at 0,1 and $\infty$. For open strings the remnant symmetry preserves the boundary is $S L(2, \mathbb{R})$. In the open string case the vertex operators are inserted on the boundary of the disk and this symmetry can be used to fix three points on the boundary that also may be 0,1 and $\infty$. As with the case of closed strings, for open strings the presence of a $B$-field spoils the $S L(2, \mathbb{R})$ symmetry avoiding the possibility to fix three points of the positions of the vertex operators on the boundary $\partial \Sigma$.

The two-point function with these boundary conditions is given by

$$
\begin{aligned}
\left\langle X^{\mu}(z) X^{v}\left(z^{\prime}\right)\right\rangle & =-\alpha^{\prime}\left[g^{\mu v} \log \left|z-z^{\prime}\right|-g^{\mu v} \log \left|z-\bar{z}^{\prime}\right|\right. \\
& \left.+G^{\mu v} \log \left|z-\bar{z}^{\prime}\right|^{2}+\frac{1}{2 \pi \alpha^{\prime}} \theta^{\mu v} \log \frac{z-\bar{z}^{\prime}}{\bar{z}-z^{\prime}}+D^{\mu v}\right]
\end{aligned}
$$

with

$$
\begin{aligned}
G^{\mu \nu} & =\left(\frac{1}{g+2 \pi \alpha^{\prime} B} g \frac{1}{g-2 \pi \alpha^{\prime} B}\right)^{\mu \nu} \\
\theta^{\mu \nu} & =-\left(2 \pi \alpha^{\prime}\right)^{2}\left(\frac{1}{g+2 \pi \alpha^{\prime} B} B \frac{1}{g-2 \pi \alpha^{\prime} B}\right)^{\mu \nu}
\end{aligned}
$$

and where $D^{\mu v}$ are quantities independent on $z$. Basically, what we want are the $N$-tachyon scattering amplitudes at tree level for the action (5). This is done by inserting the following $N$ tachyon vertex operators $\mathcal{V}(k, z)=e^{i k X(z)}$ at different points of the boundary of the open string worldsheet $\partial \Sigma$ and obtaining the correlation functions of these operators.

To obtain it we must realize that the correlators in the path integral formalism can be computed as Gaussian integrals [54]. Consider the following integral

$$
\int D X \exp \left(\int d^{2} z(X \Delta X+i J X)\right) \sim \exp \left(\frac{1}{2} \int d^{2} z d^{2} z^{\prime} J(z) K\left(z, z^{\prime}\right) J\left(z^{\prime}\right)\right),
$$

where $\Delta$ is a differential operator. The symbol $\sim$ has been used to indicate that there are some proportional factors not relevant to the analysis. Here $K$ is the inverse operator, or Green's function, that satisfies

$$
\Delta K\left(z, z^{\prime}\right)=\delta\left(z-z^{\prime}\right) .
$$

We can see that we can use this result to obtain the scattering amplitudes by choosing the appropriate source $J(z)$.

$$
\begin{aligned}
\left\langle\mathcal{V}\left(\boldsymbol{k}_{1}, z_{1}\right) \mathcal{V}\left(\boldsymbol{k}_{2}, z_{2}\right) \ldots \mathcal{V}\left(\boldsymbol{k}_{N}, z_{N}\right)\right\rangle & \\
& \sim \int D X \exp \left\{-S+i\left(\boldsymbol{k}_{1} X_{1}(\sigma)+\cdots+\boldsymbol{k}_{N} X_{N}(\sigma)\right)\right\} .
\end{aligned}
$$

Since we are interested in the vertex operators inserted at the boundary, we should restrict the propagator to it. By taking $z=\tau$ and $z^{\prime}=\tau^{\prime}$ for real $\tau, \tau^{\prime}$ in (7) we get

$$
\left\langle X^{\mu}(\tau) X^{v}\left(\tau^{\prime}\right)\right\rangle=-\alpha^{\prime} G^{\mu \nu} \log \left|\tau-\tau^{\prime}\right|+\frac{i}{2} \theta^{\mu v} \operatorname{sgn}\left(\tau-\tau^{\prime}\right),
$$

where $\operatorname{sgn}$ is the sign function. Now we can see that it suffices to choose the current as

$$
J(\tau)=\sum_{l} \delta\left(\tau-\tau_{l}\right) \boldsymbol{k}_{l}
$$


in (9). From this we can obtain the scattering amplitudes by finally integrating the expected values of the vertex operators over the entire boundary of the worldsheet, which now is just integrating over the real variable $\tau$. The result is

$$
\begin{gathered}
\int d^{N} \tau\left\langle\mathcal{V}\left(\boldsymbol{k}_{1}, \tau_{1}\right) \mathcal{V}\left(\boldsymbol{k}_{2}, \tau_{2}\right) \cdots \mathcal{V}\left(\boldsymbol{k}_{N}, \tau_{N}\right)\right\rangle \sim \int d^{N} \tau \exp \left(\frac{-i}{2} \sum_{l>m} \boldsymbol{k}^{l} \theta \boldsymbol{k}^{m} \operatorname{sgn}\left(\tau_{l}-\tau_{m}\right)\right) \\
\times \exp \left(\alpha^{\prime} \sum_{l, m} \boldsymbol{k}^{l} G \boldsymbol{k}^{m} \log \left|\tau_{l}-\tau_{m}\right|\right) \\
=\int d^{N} \tau \exp \left(\frac{-i}{2} \sum_{l>m} \boldsymbol{k}^{l} \theta \boldsymbol{k}^{m} \operatorname{sgn}\left(\tau_{l}-\tau_{m}\right)\right) \prod_{l, m}\left|\tau_{l}-\tau_{m}\right|^{\alpha^{\prime} \boldsymbol{k}^{l} G \boldsymbol{k}^{m}}
\end{gathered}
$$

where $\boldsymbol{k}^{l} \theta \boldsymbol{k}^{m}:=\boldsymbol{k}_{\mu}^{l} \theta^{\mu v} \boldsymbol{k}_{v}^{m}$ and $\boldsymbol{k}^{l} G \boldsymbol{k}^{m}:=\boldsymbol{k}_{\mu}^{l} G^{\mu v} \boldsymbol{k}_{v}^{m}$. These amplitudes are of the KobaNielsen type. The factor involving the sign function in the last line is actually more general, because it only depends on the variables through the sign functions, and any derivatives of it are 0 . This allows us to write

$$
\begin{aligned}
& \left\langle\prod_{l=1}^{N} P_{l}\left(\partial X\left(\tau_{l}\right), \partial^{2} X\left(\tau_{l}\right) \ldots\right) e^{i k_{l} X\left(\tau_{l}\right)}\right\rangle_{G, \theta} \\
& =e^{\frac{i}{2} \sum_{l>m} k_{\mu}^{l} \theta^{\nu v} k_{\nu}^{m} \operatorname{sgn}\left(\tau_{l}-\tau_{m}\right)}\left\langle\prod_{l=1}^{N} P_{l}\left(\partial X\left(\tau_{l}\right), \partial^{2} X\left(\tau_{l}\right) \ldots\right) e^{i \boldsymbol{k}_{l} X\left(\tau_{l}\right)}\right\rangle_{G, \theta=0} .
\end{aligned}
$$

The symbol $\langle\cdots\rangle_{G, \theta}=0$ means that the expectation value taken on the second line is using the propagator (10) without the second term. This is achieved by writing the polynomial as an exponential and keeping only the corresponding linear terms. For example [48]:

$$
\mathcal{V}(\zeta, k, z)=i \zeta^{\mu} \partial X^{\mu} e^{i k X(z)}=\left[\exp \left(i k X+i \zeta^{\mu} \partial X^{\mu}\right)\right]_{\text {linear }}
$$

where $[\cdots]_{\text {linear }}$ indicates to only keep linear terms in $\zeta$. So, we can see two direct effects of the $B$-field on the action (5). The first is that the effective metric on spacetime becomes $G_{\mu \nu}$ given in (8). The second is that at tree level (Remember, this came from the fact that we are in the full upper half plane with the simplest zero genus topology) the scattering amplitudes acquire a phase factor that depends on the cyclic ordering of the momenta $k^{\mu}$ and the matrix $\theta$. But the overall form of the amplitudes is the same as without the $B$-field.

In the case of open strings for $N$ points with vanishing $B$-field and with flat metric $G_{\mu v}=\eta_{\mu v}$ and units with $\alpha^{\prime}=1$ and for a fixed ordering of the external momenta $\boldsymbol{k}_{i}$, from (11) we obtain the Koba-Nielsen amplitude, with the three fixed points in $x_{1}=0$, $x_{N-1}=1$ and $x_{N}=\infty$,

$$
\begin{aligned}
\widetilde{A}_{\mathbb{R}}^{(N)}(\boldsymbol{k})=\int_{0<x_{2}<x_{3}<\cdots<x_{N-2}<1} d x_{2} d x_{3} \cdots d x_{N-2} \prod_{j=2}^{N-2}\left|x_{j}\right|^{k_{1} k_{j}} \mid 1 & -\left.x_{j}\right|^{k_{N-1} k_{j}} \\
& \times \prod_{2 \leq i<j \leq N-2}\left|x_{i}-x_{j}\right|^{k_{i} \boldsymbol{k}_{j}},
\end{aligned}
$$

where we have changed the variables $\tau$ to $x^{\prime}$ s variables in (11), In [48,53-55].

The four-point amplitude is Veneziano's amplitude

$$
\widetilde{A}_{\mathbb{R}}^{(4)}(\boldsymbol{k})=\int_{0}^{1} d x|x|^{k_{1} k_{2}}|1-x|^{k_{2} k_{3}} .
$$

It is known that this definition of Veneziano's amplitude describes only one of the three decay channels $s, t$ and $u$ corresponding to the different inequivalent permutations of 
the momenta. If we consider the sum over the three channels and performing a suitable change of variables [59] is possible to write the total amplitude as

$$
A_{\mathbb{R}}^{(4)}(\boldsymbol{k})=\int_{\mathbb{R}} d x|x|^{k_{1} k_{2}}|1-x|^{k_{2} k_{3}} .
$$

In terms of the gamma function the above amplitude writes

$$
\begin{aligned}
A_{\mathbb{R}}^{(4)}(\boldsymbol{k})=\frac{\Gamma\left(-1-\alpha^{\prime} s\right) \Gamma\left(-1-\alpha^{\prime} t\right)}{\Gamma\left(-2-\alpha^{\prime}(s+t)\right)}+\frac{\Gamma\left(-1-\alpha^{\prime} s\right) \Gamma\left(-1-\alpha^{\prime} u\right)}{\Gamma\left(-2-\alpha^{\prime}(s+u)\right)} & +\frac{\Gamma\left(-1-\alpha^{\prime} t\right) \Gamma\left(-1-\alpha^{\prime} u\right)}{\Gamma\left(-2-\alpha^{\prime}(t+u)\right)}
\end{aligned}
$$

where $s, t$ and $u$ are the Mandelstam variables and they are defined as $s:=-\left(p_{1}+p_{2}\right)^{2}$, $t:=-\left(p_{1}+p_{3}\right)^{2}$ and $u:=-\left(p_{1}+p_{4}\right)^{2}$ and they satisfy $s+t+u=-\sum_{i} \boldsymbol{k}_{i}^{2}=\sum_{i} M_{i}^{2}$, and

$$
\Gamma(u)=\int_{0}^{\infty} d t t^{u-1} e^{-t}
$$

is the gamma function. Thus, the 4-point amplitudes whose integration is $\mathbb{R}$ it already includes the three channels.

Moreover, this procedure also works for $N$ point amplitudes $[4,48]$ and it yields

$$
\begin{aligned}
A_{\mathbb{R}}^{(N)}(\boldsymbol{k})=\int_{\mathbb{R}^{N-3}} d x_{2} d x_{3} \cdots d x_{N-2} \prod_{j=2}^{N-2}\left|x_{j}\right|^{k_{1} k_{j}}\left|1-x_{j}\right|^{k_{N-1} k_{j}} & \\
& \times \prod_{2 \leq i<j \leq N-2}\left|x_{i}-x_{j}\right|^{k_{i} k_{j} .}
\end{aligned}
$$

For completeness we write the form of closed strings in absence of $B$-field the $N$-point amplitude is given by

$$
A_{\mathbb{C}}^{(N)}(\boldsymbol{k})=\int_{\mathbb{C}^{N-2}} \prod d^{2} z_{i} \prod_{i<j}\left|z_{i}-z_{j}\right|^{\boldsymbol{k}_{i} \boldsymbol{k}_{j} / 2} .
$$

The four-point amplitude is the Virasoro-Shapiro amplitude

$$
\begin{aligned}
A_{\mathbb{C}}^{(4)}(\boldsymbol{k}) & =\int_{\mathbb{C}^{2}} d^{2} z|z|^{k_{1} k_{4} / 2}|1-z|^{k_{2} k_{4} / 2} \\
& =\frac{\Gamma\left(-1-\frac{\alpha^{\prime} s}{4}\right) \Gamma\left(-1-\frac{\alpha^{\prime} t}{4}\right) \Gamma\left(-1-\frac{\alpha^{\prime} u}{4}\right)}{\Gamma\left(2+\frac{\alpha^{\prime} s}{4}\right) \Gamma\left(2+\frac{\alpha^{\prime} t}{4}\right) \Gamma\left(2+\frac{\alpha^{\prime} u}{4}\right)} .
\end{aligned}
$$

It is worth noting that the Veneziano four-point amplitude (12), describing only one channel, can be written also as an integral over the whole $\mathbb{R}$ if we introduce some multiplicative characters as $\operatorname{sgn}(x)$ :

$$
\widetilde{A}_{\mathbb{R}}^{(4)}(\boldsymbol{k})=\frac{1}{2} \int_{\mathbb{R}} d x|x|^{k_{1} k_{2}}|1-x|^{k_{2} k_{3}}+\frac{1}{2} \int_{\mathbb{R}} d x|x|^{k_{1} k_{2}}|1-x|^{k_{2} k_{3}} \operatorname{sgn}(x) \operatorname{sgn}(1-x),
$$

where the function $\operatorname{sign}(x)$ is +1 of $x>0$ and is -1 if $x<0$ [14].

The generalization for $N$-point amplitudes is given by

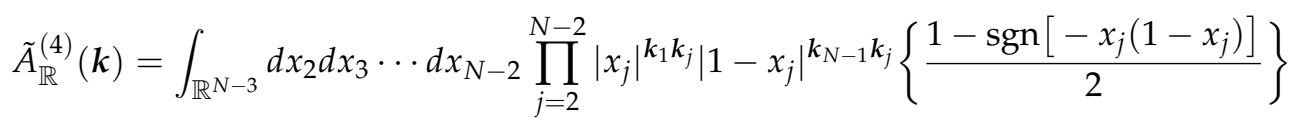




$$
\times \prod_{2 \leq i<j \leq N-2}\left|x_{i}-x_{j}\right|^{k_{i} k_{j}} \frac{1}{2}\left\{1-\operatorname{sgn}\left[(-1)\left(x_{i}-x_{j}\right)\right]\right\},
$$

In [14]. The open string amplitude (11) with a non-vanishing $B$-field can be carried out in this form using the multiplicative character $\operatorname{sign}(x)$. This form is of particular interest in connection to the Ghoshal-Kawano amplitudes [60] studied later in this survey.

\section{Essential Ideas of $p$-Adic Analysis}

In this section, we review some ideas and results on $p$-adic analysis that we will use throughout this article. For an in-depth exposition, the reader may consult $[3,61,62]$.

\subsection{The Field of $p$-Adic Numbers}

Throughout this article $p$ will denote a prime number. The field of $p$-adic numbers $\mathbb{Q}_{p}$ is defined as the completion of the field of rational numbers $\mathbb{Q}$ with respect to the $p$-adic norm $|\cdot|_{p}$, which is defined as

$$
|x|_{p}=\left\{\begin{array}{lll}
0 & \text { if } & x=0 \\
p^{-\gamma} & \text { if } & x=p^{\gamma} \frac{a}{b}
\end{array}\right.
$$

where $a$ and $b$ are integers coprime with $p$. The integer $\gamma:=\operatorname{ord}(x)$, with $\operatorname{ord}(0):=+\infty$, is called the $p$-adic order of $x$. We extend the $p$-adic norm to $\mathbb{Q}_{p}^{n}$ by taking

$$
\|x\|_{p}:=\max _{1 \leq i \leq n}\left|x_{i}\right|_{p}, \quad \text { for } x=\left(x_{1}, \ldots, x_{n}\right) \in \mathbb{Q}_{p}^{n} .
$$

We define $\operatorname{ord}(\boldsymbol{x})=\min _{1 \leq i \leq n}\left\{\operatorname{ord}\left(x_{i}\right)\right\}$, then $\|x\|_{p}=p^{-\operatorname{ord}(x)}$. The metric space $\left(\mathbb{Q}_{p}^{n},\|\cdot\|_{p}\right)$ is a complete ultrametric space. As a topological space $\mathbb{Q}_{p}$ is homeomorphic to a Cantor-like subset of the real line, see e.g., [3,61].

Any $p$-adic number $x \neq 0$ has a unique expansion of the form

$$
x=p^{\operatorname{ord}(x)} \sum_{i=0}^{\infty} x_{i} p^{i}
$$

where $x_{i} \in\{0,1,2, \ldots, p-1\}$ and $x_{0} \neq 0$.

For $r \in \mathbb{Z}$, denote by $B_{r}^{n}(\boldsymbol{a})=\left\{\boldsymbol{x} \in \mathbb{Q}_{p}^{n} ;\|\boldsymbol{x}-\boldsymbol{a}\|_{p} \leq p^{r}\right\}$ the ball of radius $p^{r}$ with center at $\boldsymbol{a}=\left(a_{1}, \ldots, a_{n}\right) \in \mathbb{Q}_{p}^{n}$, and take $B_{r}^{n}(\mathbf{0}):=B_{r}^{n}$. Please note that $B_{r}^{n}(\boldsymbol{a})=B_{r}\left(a_{1}\right) \times \cdots \times B_{r}\left(a_{n}\right)$, where $B_{r}\left(a_{i}\right):=\left\{x \in \mathbb{Q}_{p} ;\left|x_{i}-a_{i}\right|_{p} \leq p^{r}\right\}$ is the one-dimensional ball of radius $p^{r}$ with center at $a_{i} \in \mathbb{Q}_{p}$. The ball $B_{0}^{n}$ equals the product of $n$ copies of $B_{0}=\mathbb{Z}_{p}$, the ring of $p$-adic integers. In addition, $B_{r}^{n}(\boldsymbol{a})=\boldsymbol{a}+\left(p^{-r} \mathbb{Z}_{p}\right)^{n}$. We also denote by $S_{r}^{n}(\boldsymbol{a})=\left\{\boldsymbol{x} \in \mathbb{Q}_{p}^{n} ;\|\boldsymbol{x}-\boldsymbol{a}\|_{p}=p^{r}\right\}$ the sphere of radius $p^{r}$ with center at $\boldsymbol{a} \in \mathbb{Q}_{p}^{n}$, and take $S_{r}^{n}(\mathbf{0}):=S_{r}^{n}$. We notice that $S_{0}^{1}=\mathbb{Z}_{p}^{\times}$ (the group of units of $\mathbb{Z}_{p}$ ), but $\left(\mathbb{Z}_{p}^{\times}\right)^{n} \subsetneq S_{0}^{n}$. The balls and spheres are both open and closed subsets in $\mathbb{Q}_{p}^{n}$. In addition, two balls in $\mathbb{Q}_{p}^{n}$ are either disjoint or one is contained in the other.

As a topological space $\left(\mathbb{Q}_{p}^{n},\|\cdot\|_{p}\right)$ is totally disconnected, i.e., the only connected subsets of $\mathbb{Q}_{p}^{n}$ are the empty set and the points. A subset of $\mathbb{Q}_{p}^{n}$ is compact if and only if it is closed and bounded in $\mathbb{Q}_{p}^{n}$, see e.g., ([3], Section 1.3), or ([61], Section 1.8). The balls and spheres are compact subsets. Thus, $\left(\mathbb{Q}_{p}^{n},\|\cdot\|_{p}\right)$ is a locally compact topological space.

Remark 1. There is a natural map, called the reduction $\bmod p$ and denoted as $\bar{i}$, from $\mathbb{Z}_{p}$ onto $\mathbb{F}_{p}$, the finite field with $p$ elements. More precisely, if $x=\sum_{j=0}^{\infty} x_{j} p^{j} \in \mathbb{Z}_{p}$, then $\bar{x}=x_{0} \in \mathbb{F}_{p}=$ $\{0,1, \ldots, p-1\}$. If $\boldsymbol{a}=\left(a_{1}, \ldots, a_{n}\right) \in \mathbb{Z}_{p}^{n}$, then $\overline{\boldsymbol{a}}=\left(\bar{a}_{1}, \ldots, \bar{a}_{n}\right)$. 


\subsection{Integration on $\mathbb{Q}_{p}^{n}$}

Since $\left(\mathbb{Q}_{p},+\right)$ is a locally compact topological group, there exists a Borel measure $d x$, called the Haar measure of $\left(\mathbb{Q}_{p},+\right)$, unique up to multiplication by a positive constant, such that $\int_{U} d x>0$ for every nonempty Borel open set $U \subset \mathbb{Q}_{p}$, and satisfying $\int_{E+z} d x=\int_{E} d x$ for every Borel set $E \subset \mathbb{Q}_{p}$, see e.g., ([63], Chapter XI). If we normalize this measure by the condition $\int_{\mathbb{Z}_{p}} d x=1$, then $d x$ is unique. From now on we denote by $d x$ the normalized Haar measure of $\left(\mathbb{Q}_{p},+\right)$ and by $d^{n} x$ the product measure on $\left(\mathbb{Q}_{p}^{n},+\right)$.

A function $\varphi: \mathbb{Q}_{p}^{n} \rightarrow \mathbb{C}$ is said to be locally constant if for every $x \in \mathbb{Q}_{p}^{n}$ there exists an open compact subset $U, x \in U$, such that $\varphi(\boldsymbol{x})=\varphi(\boldsymbol{u})$ for all $\boldsymbol{u} \in U$. Any locally constant function $\varphi: \mathbb{Q}_{p}^{n} \rightarrow \mathbb{C}$ can be expressed as a linear combination of characteristic functions of the form $\varphi(x)=\sum_{k=1}^{\infty} c_{k} 1_{U_{k}}(x)$, where $c_{k} \in \mathbb{C}$ and $1_{U_{k}}(\boldsymbol{x})$ is the characteristic function of $U_{k}$, an open compact subset of $\mathbb{Q}_{p}^{n}$, for every $k$. If $\varphi$ has compact support, then $\varphi(x)=\sum_{k=1}^{L} c_{k} 1_{U_{k}}(\boldsymbol{x})$ and in this case

$$
\int_{\mathbb{Q}_{p}^{n}} \varphi(x) d^{n} x=c_{1} \int_{U_{1}} d^{n} x+\ldots+c_{L} \int_{U_{L}} d^{n} x .
$$

A locally constant function with compact support is called a Bruhat-Schwartz function. These functions form a $\mathbb{C}$-vector space denoted as $\mathcal{D}\left(\mathbb{Q}_{p}^{n}\right)$. By using the fact that $\mathcal{D}\left(\mathbb{Q}_{p}^{n}\right)$ is a dense subspace of $C_{c}\left(\mathbb{Q}_{p}^{n}\right)$, the $\mathbb{C}$-space of continuous functions on $\mathbb{Q}_{p}^{n}$ with compact support, with the topology of the uniform convergence, the functional $\varphi \rightarrow \int_{\mathbb{Q}_{p}^{n}} \varphi(x) d^{n} \boldsymbol{x}$, $\varphi \in \mathcal{D}\left(\mathbb{Q}_{p}^{n}\right)$ has a unique continuous extension to $C_{c}\left(\mathbb{Q}_{p}^{n}\right)$, as an unbounded linear functional. For integrating more general functions, say locally integrable functions, the following notion of improper integral will be used.

Definition 1. A function $\varphi \in L_{\text {loc }}^{1}$ is said to be integrable in $\mathbb{Q}_{p}^{n}$ if

$$
\lim _{m \rightarrow+\infty} \int_{B_{m}^{n}} \varphi(x) d^{n} x=\lim _{m \rightarrow+\infty} \sum_{j=-\infty}^{m} \int_{S_{j}^{n}} \varphi(x) d^{n} \boldsymbol{x}
$$

exists. If the limit exists, it is denoted as $\int_{\mathbb{Q}_{p}^{n}} \varphi(x) d^{n} \boldsymbol{x}$, and we say that the (improper) integral exists.

\subsection{Analytic Change of Variables}

A function $h: U \rightarrow \mathbb{Q}_{p}$ is said to be analytic on an open subset $U \subset \mathbb{Q}_{p}^{n}$, if for every $\boldsymbol{b} \in U$ there exists an open subset $\widetilde{U} \subset U$, with $\boldsymbol{b} \in \widetilde{U}$, and a convergent power series $\sum_{i} a_{i}(\boldsymbol{x}-\boldsymbol{b})^{i}$ for $x \in \widetilde{U}$, such that $h(\boldsymbol{x})=\sum_{i \in \mathbb{N}^{n}} a_{i}(\boldsymbol{x}-\boldsymbol{b})^{i}$ for $x \in \widetilde{U}$, with $\boldsymbol{x}^{i}=x_{1}^{i_{1}} \cdots x_{n}^{i_{n}}$, $\boldsymbol{i}=\left(i_{1}, \ldots, i_{n}\right)$. In this case, $\frac{\partial}{\partial x_{l}} h(\boldsymbol{x})=\sum_{i \in \mathbb{N}^{n}} a_{i} \frac{\partial}{\partial x_{l}}(\boldsymbol{x}-\boldsymbol{b})^{i}$ is a convergent power series. Let $U, V$ be open subsets of $\mathbb{Q}_{p}^{n}$. A mapping $\boldsymbol{h}: U \rightarrow V, \boldsymbol{h}=\left(h_{1}, \ldots, h_{n}\right)$ is called analytic if each $h_{i}$ is analytic.

Let $\varphi: V \rightarrow \mathbb{C}$ be a continuous function with compact support, and let $\boldsymbol{h}: U \rightarrow V$ be an analytic mapping. Then

$$
\int_{V} \varphi(\boldsymbol{y}) d^{n} \boldsymbol{y}=\int_{U} \varphi(\boldsymbol{h}(\boldsymbol{x}))|\operatorname{Jac}(\boldsymbol{h}(\boldsymbol{x}))|_{p} d^{n} \boldsymbol{x},
$$

where $\operatorname{Jac}(\boldsymbol{h}(\boldsymbol{z})):=\operatorname{det}\left[\frac{\partial h_{i}}{\partial x_{j}}(\boldsymbol{z})\right]_{\substack{1 \leq i \leq n \\ 1 \leq j \leq n}}$, see e.g., ([64], Section 10.1.2).

Example 1. In dimension one, the change of variables Formula (13) implies that

$$
\int_{a U} d x=|a|_{p} \int_{U} d y
$$


for any $a \in \mathbb{Q}_{p}^{\times}$and any Borel set $U \subseteq \mathbb{Q}_{p}$. For example,

$$
\int_{B_{r}} d x=\int_{p^{-r} \mathbb{Z}_{p}} d x=\left|p^{-r}\right|_{p} \int_{\mathbb{Z}_{p}} d y=p^{r} \int_{\mathbb{Z}_{p}} d y=p^{r},\left(x=p^{-r} y\right)
$$

and

$$
\int_{S_{r}} d x=\int_{B_{r}} d x-\int_{B_{r-1}} d x=p^{r}-p^{r-1}=p^{r}\left(1-p^{-1}\right) .
$$

Example 2. We now compute the following integral

$$
\mathrm{Z}(s)=\int_{\mathbb{Z}_{p}}|x|_{p}^{s} d x, s \in \mathbb{C} \text {, with } \operatorname{Re}(s)>0 \in \mathbb{C} .
$$

We use the partition $\mathbb{Z}_{p}=p \mathbb{Z}_{p} \sqcup \mathbb{Z}_{p}^{\times}$, where $\mathbb{Z}_{p}^{\times}$is the group of units of $\mathbb{Z}_{p}$ :

$$
\mathbb{Z}_{p}^{\times}:=\left\{x \in \mathbb{Z}_{p} ;|x|_{p}=1\right\}=\left\{\sum_{i=0}^{\infty} x_{i} p^{i} \in \mathbb{Z}_{p} ; x_{0} \neq 0\right\} .
$$

Using (15),

$$
\begin{aligned}
Z(s) & =\int_{p \mathbb{Z}_{p}}|x|_{p}^{s} d x+\int_{\mathbb{Z}_{p}^{\times}}|x|_{p}^{s} d x=p^{-1-s} \int_{\mathbb{Z}_{p}}|x|_{p}^{s} d x+\int_{\mathbb{Z}_{p}^{\times}} d x \\
& =p^{-1-s} \int_{\mathbb{Z}_{p}}|x|_{p}^{s} d x+\left(1-p^{-1}\right)=p^{-1-s} Z(s)+\left(1-p^{-1}\right) .
\end{aligned}
$$

Therefore,

$$
Z(s)=\frac{1-p^{-1}}{1-p^{-1-s}} \text { for } \operatorname{Re}(s)>-1 .
$$

Example 3. We now consider the integral

$$
Z(s):=\int_{\mathbb{Z}_{p}^{\times}}|1-x|_{p}^{s} d x, s \in \mathbb{C} \text {, with } \operatorname{Re}(s)>0 \in \mathbb{C} .
$$

Assuming that $\mathbb{Z}_{p}^{\times}=\sqcup_{a_{0} \in \mathbb{F}_{p}^{\times}}\left(a_{0}+p \mathbb{Z}_{p}\right)$, where $\mathbb{F}_{p}^{\times}=\{1,2, \ldots, p-1\}$, and (16) we have

$$
\begin{aligned}
Z(s)=\int_{1+p \mathbb{Z}_{p}}|1-x|_{p}^{s} d x & +\sum_{a_{0}=2}^{p-1} \int_{a_{0}+p \mathbb{Z}_{p}}|1-x|_{p}^{s} d x=p^{-1-s} \int_{\mathbb{Z}_{p}}|y|_{p}^{s} d y \\
& +p^{-1} \sum_{a_{0}=2}^{p-1} \int_{\mathbb{Z}_{p}}\left|1-\left(a_{0}+p y\right)\right|_{p}^{s} d y=p^{-1-s} \frac{1-p^{-1}}{1-p^{-1-s}}+p^{-1}(p-2) .
\end{aligned}
$$

\subsection{Some p-Adic Integrals}

In this section, we compute some integrals that we will use later on.

Example 4. We consider the following integral:

$$
Z(s):=\int_{\mathbb{Z}_{p} \times \mathbb{Z}_{p}}|x-y|_{p}^{s} d x d y, s \in \mathbb{C} \text {, with } \operatorname{Re}(s)>0 \in \mathbb{C} .
$$



we have

Using the partition $\mathbb{Z}_{p}^{2}=\left(p \mathbb{Z}_{p}\right)^{2} \sqcup S_{0}^{2}$, with $S_{0}^{2}=p \mathbb{Z}_{p} \times \mathbb{Z}_{p}^{\times} \sqcup \mathbb{Z}_{p}^{\times} \times p \mathbb{Z}_{p} \sqcup \mathbb{Z}_{p}^{\times} \times \mathbb{Z}_{p}^{\times}$,

$$
\begin{aligned}
Z(s)= & \int_{\left(p \mathbb{Z}_{p}\right)^{2}}|x-y|_{p}^{s} d x d y+\int_{p \mathbb{Z}_{p} \times \mathbb{Z}_{p}^{\times}}|x-y|_{p}^{s} d x d y+\int_{\mathbb{Z}_{p}^{\times} \times p \mathbb{Z}_{p}}|x-y|_{p}^{s} d x d y \\
& +\int_{\mathbb{Z}_{p}^{\times} \times \mathbb{Z}_{p}^{\times}}|x-y|_{p}^{s} d x d y=p^{-2-s} Z(s)+p^{-1} \int_{\mathbb{Z}_{p} \times \mathbb{Z}_{p}^{\times}} d x d y+p^{-1} \int_{\mathbb{Z}_{p}^{\times} \times \mathbb{Z}_{p}} d x d y \\
& +\int_{\mathbb{Z}_{p}^{\times} \times \mathbb{Z}_{p}^{\times}}|x-y|_{p}^{s} d x d y .
\end{aligned}
$$

To compute the last integral, we use the partition

$$
\left(\mathbb{Z}_{p}^{\times}\right)^{2}=\bigsqcup_{a_{0}, a_{1} \in \mathbb{F}_{p}^{\times}}\left(a_{0}+p \mathbb{Z}_{p}\right) \times\left(a_{1}+p \mathbb{Z}_{p}\right)
$$

as follows:

$$
\begin{gathered}
\int_{\substack{\mathbb{Z}_{p}^{\times} \times \mathbb{Z}_{p}^{\times}\\
}}|x-y|_{p}^{s} d x d y=\sum_{\bar{a}_{0}, \bar{a}_{1} \in \mathbb{F}_{p}^{\times}} \int_{\left(a_{0}+p \mathbb{Z}_{p}\right) \times\left(a_{1}+p \mathbb{Z}_{p}\right)}|x-y|_{p}^{s} d x d y \\
\sum_{\substack{a_{0}, a_{1} \in \mathbb{F}_{p}^{\times} \\
a_{0} \neq a_{1}}} \int_{\mathbb{Z}_{p} \times \mathbb{Z}_{p}}\left|a_{0}+p x-a_{1}-p y\right|_{p}^{s} d x d y+p^{-2-s} \sum_{\substack{a_{0}, a_{1} \in \mathbb{F}_{p}^{\times} \\
a_{0}=a_{1}}} \int_{\mathbb{Z}_{p} \times \mathbb{Z}_{p}}|x-y|_{p}^{s} d x d y \\
=p^{-2}(p-1)(p-2)+p^{-2-s}(p-1) Z(s) .
\end{gathered}
$$

Hence,

$$
Z(s)=\frac{1-p^{-1}}{1-p^{-1-s}}
$$

Example 5. We now show that

$$
Z(s):=\int_{\mathbb{Z}_{p}^{\times} \times \mathbb{Z}_{p}^{\times}}|x-y|_{p}^{s} d x d y=p^{-2}(p-1)(p-2)+p^{-2-s}(p-1) \frac{1-p^{-1}}{1-p^{-1-s}} .
$$

Indeed, by changing variables as $x=u v, y=v$,

$$
Z(s)=\int_{\mathbb{Z}_{p}^{\times} \times \mathbb{Z}_{p}^{\times}}|u-1|_{p}^{s} d u d v=\left(1-p^{-1}\right) \int_{\mathbb{Z}_{p}^{\times}}|u-1|_{p}^{s} d u .
$$

Now the result follows from Example 3.

Example 6. We now compute the following integral

$$
Z\left(s_{1}, s_{2}, s_{3}\right):=\int_{\mathbb{Z}_{p}^{\times} \times \mathbb{Z}_{p}^{\times}}|1-x|_{p}^{s_{1}}|1-y|_{p}^{s_{2}}|x-y|_{p}^{s_{3}} d x d y,
$$

where $s_{1}, s_{2}, s_{3} \in \mathbb{C}$ satisfying $\operatorname{Re}\left(s_{1}\right), \operatorname{Re}\left(s_{2}\right), \operatorname{Re}\left(s_{3}\right)>0$. By using the partition

$$
\left(\mathbb{Z}_{p}^{\times}\right)^{2}=\underset{a_{0}, a_{1} \in \mathbb{F}_{p}^{\times}}{\sqcup}\left(a_{0}+p \mathbb{Z}_{p}\right) \times\left(a_{1}+p \mathbb{Z}_{p}\right),
$$

where $\mathbb{F}_{p}^{\times}=\{1,2, \ldots, p-1\}$, 


$$
\begin{aligned}
Z\left(s_{1}, s_{2}, s_{3}\right) & =\sum_{a_{0}=1}^{p-1} \sum_{a_{1}=1}^{p-1} \int_{\left(a_{0}+p \mathbb{Z}_{p}\right) \times\left(a_{1}+p \mathbb{Z}_{p}\right)}|1-x|_{p}^{s_{1}}|1-y|_{p}^{s_{2}}|x-y|_{p}^{s_{3}} d x d y \\
& =: \sum_{a_{0}=1}^{p-1} \sum_{a_{1}=1}^{p-1} J_{a_{0}, a_{1}}\left(s_{1}, s_{2}, s_{3}\right) .
\end{aligned}
$$

By changing variables as $x=a_{0}+p u, y=a_{1}+p v$ we have

$$
J_{a_{0}, a_{1}}\left(s_{1}, s_{2}, s_{3}\right)=p^{-2} \int_{\mathbb{Z}_{p}^{2}}\left|1-\left(a_{0}+p u\right)\right|_{p}^{s_{1}}\left|1-\left(a_{1}+p v\right)\right|_{p}^{s_{2}}\left|\left(a_{0}+p u\right)-\left(a_{1}+p v\right)\right|_{p}^{s_{3}} d u d v .
$$

Now, we compute the integrals $J_{a_{0}, a_{1}}$. This calculation involves several cases.

Case 1. If $a_{0} \neq 1, a_{1} \neq 1 \quad a_{0} \neq a_{1}$, then $\left|1-\left(a_{0}+p u\right)\right|_{p}=1,\left|1-\left(a_{1}+p v\right)\right|_{p}=1$, and $\left|\left(a_{0}+p u\right)-\left(a_{1}+p v\right)\right|_{p}=1$. and

$$
J_{a_{0}, a_{1}}\left(s_{1}, s_{2}, s_{3}\right)=p^{-2} \int_{\mathbb{Z}_{p}^{2}} d u d v=p^{-2} .
$$

There are $(p-2)(p-3)$ integrals of this type.

Case 2. If $a_{0} \neq 1, a_{1} \neq 1 a_{0}=a_{1}$, then, $\left|1-\left(a_{0}+p u\right)\right|_{p}=1,\left|1-\left(a_{1}+p v\right)\right|_{p}=1$. By Example (17),

$$
J_{a_{0}, a_{1}}\left(s_{1}, s_{2}, s_{3}\right)=p^{-2-s_{3}} \int_{\mathbb{Z}_{p} \times \mathbb{Z}_{p}}|u-v|_{p}^{s_{3}} d u d v=\frac{p^{-2-s_{3}}\left(1-p^{-1}\right)}{1-p^{-1-s_{3}}},
$$

for $\operatorname{Re}\left(s_{3}\right)>-1$. There are $p-2$ integrals of this type.

Case 3. If $a_{0}=1, a_{1} \neq 1$, then $\left|1-\left(a_{1}+p v\right)\right|_{p}=1,\left|\left(a_{0}+p u\right)-\left(a_{1}+p v\right)\right|_{p}=1$. By using (16)

$$
J_{a_{0}, a_{1}}\left(s_{1}, s_{2}, s_{3}\right)=p^{-2-s_{1}} \int_{\mathbb{Z}_{p}^{2}}|u|_{p}^{s_{1}} d u d v=\frac{p^{-2-s_{1}}\left(1-p^{-1}\right)}{1-p^{-1-s_{1}}},
$$

for $\operatorname{Re}\left(s_{1}\right)>-1$. There are $p-2$ integrals of this type.

Case 4. If $a_{0} \neq 1, a_{1}=1$, this case is similar to Case 3 ,

$$
J_{a_{0}, a_{1}}\left(s_{1}, s_{2}, s_{3}\right)=p^{-2-s_{2}} \int_{\mathbb{Z}_{p}}\left|x_{3}\right|_{p}^{s_{2}} d x_{3}=\frac{p^{-2-s_{2}}\left(1-p^{-1}\right)}{1-p^{-1-s_{2}}}
$$

for $\operatorname{Re}\left(s_{2}\right)>-1$. Therefore

$$
\begin{aligned}
Z\left(s_{1}, s_{2}, s_{3}\right) & =p^{-2}(p-2)(p-3)+(p-2) \frac{p^{-2-s_{3}}\left(1-p^{-1}\right)}{1-p^{-1-s_{3}}} \\
& +(p-2) \frac{p^{-2-s_{1}}\left(1-p^{-1}\right)}{1-p^{-1-s_{1}}}+(p-2) \frac{p^{-2-s_{2}}\left(1-p^{-1}\right)}{1-p^{-1-s_{2}}} .
\end{aligned}
$$

on the region of $\mathbb{C}^{3}$ defined by $\operatorname{Re}\left(s_{1}\right)>-1, \operatorname{Re}\left(s_{2}\right)>-1$, and $\operatorname{Re}\left(s_{3}\right)>-1$. 


\section{4. $p$-Adic Open String Amplitudes}

In [14], Brekke, Freund, Olson and Witten work out the $N$-point amplitudes in explicit form and investigate how these can be obtained from an effective Lagrangian. The $p$-adic open string $\mathrm{N}$-point tree amplitudes are defined as

$$
\boldsymbol{A}^{(N)}(\boldsymbol{k})=\int_{\mathbb{Q}_{p}^{N-3}} \prod_{i=2}^{N-2}\left|x_{i}\right|_{p}^{k_{1} k_{i}}\left|1-x_{i}\right|_{p}^{k_{N-1} k_{i}} \prod_{2 \leq i<j \leq N-2}\left|x_{i}-x_{j}\right|_{p}^{k_{i} k_{j}} \prod_{i=2}^{N-2} d x_{i},
$$

where $\prod_{i=2}^{N-2} d x_{i}$ is the normalized Haar measure of $\mathbb{Q}_{p}^{N-3}, \boldsymbol{k}=\left(\boldsymbol{k}_{1}, \ldots, \boldsymbol{k}_{N}\right), \boldsymbol{k}_{i}=\left(k_{0, i}, \ldots, k_{l, i}\right)$, $i=1, \ldots, N, N \geq 4$, is the momentum vector of the $i$-th tachyon (with Minkowski product $\left.\boldsymbol{k}_{i} \boldsymbol{k}_{j}=-k_{0, i} k_{0, j}+k_{1, i} k_{1, j}+\cdots+k_{l, i} k_{l, j}\right)$ obeying

$$
\sum_{i=1}^{N} \boldsymbol{k}_{i}=\mathbf{0}, \quad \boldsymbol{k}_{i} \boldsymbol{k}_{i}=2 \text { for } i=1, \ldots, N .
$$

The parameter $l$ is an arbitrary positive integer. Typically, $l$ is taken to be 25 . However, we do not require using the critical dimension. In [8], we show that the $p$-adic open string amplitudes (19) are bona fide integrals and that they have meromorphic continuations as rational functions in the kinematic parameters. We attach to these amplitudes a multivariate Igusa-type zeta function. Fix an integer $N \geq 4$ and set $T:=\{2, \ldots, N-2\}$, $D=\frac{(N-3)(N-4)}{2}+2(N-3)$ and $\mathbb{C}^{D}$ as

$$
\begin{cases}\left\{s_{i j} \in \mathbb{C} ; i \in\{1, N-1\}, j \in T\right\} & \text { if } \quad N=4 \\ \left\{s_{i j} \in \mathbb{C} ; i \in\{1, N-1\}, j \in T \text { or } i, j \in T \text { with } i<j\right\} & \text { if } \quad N \geq 5 .\end{cases}
$$

We now put $s=\left(s_{i j}\right) \in \mathbb{C}^{D}$ and $\boldsymbol{k}_{i} \boldsymbol{k}_{j}=s_{i j} \in \mathbb{C}$ satisfying $s_{i j}=s_{j i}$ for $1 \leq i<j \leq N-1$. The $p$-adic open string $N$-point zeta function is defined as

$$
\boldsymbol{Z}^{(N)}(\boldsymbol{s})=\int_{\mathbb{Q}_{p}^{N-3} \backslash \Lambda} \prod_{i=2}^{N-2}\left|x_{i}\right|_{p}^{s_{1 i}}\left|1-x_{i}\right|_{p}^{s_{(N-1) i}} \prod_{2 \leq i<j \leq N-2}\left|x_{i}-x_{j}\right|_{p}^{s_{i j}} \prod_{i=2}^{N-2} d x_{i},
$$

where $s=\left(s_{i j}\right) \in \mathbb{C}^{D}, \prod_{i=2}^{N-2} d x_{i}$ is the normalized Haar measure of $\mathbb{Q}_{p}^{N-3}$, and

$$
\Lambda:=\left\{\left(x_{2}, \ldots, x_{N-2}\right) \in \mathbb{Q}_{p}^{N-3} ; \prod_{i=2}^{N-2} x_{i}\left(1-x_{i}\right) \prod_{2 \leq i<j \leq N-2}\left(x_{i}-x_{j}\right)=0\right\} .
$$

In the definition of integral (20) we remove the set $\Lambda$ from the domain of integration to use the formula $a^{s}=e^{s \ln a}$ for $a>0$ and $s \in \mathbb{C}$. The reason for using the name 'zeta function' is that the integral (20) is a finite sum of multivariate local zeta functions.

Take $\phi\left(x_{2}, \ldots, x_{N-2}\right)$ a locally constant function with compact support, then

$$
Z_{\phi}^{(N)}(s)=\int_{\mathbb{Q}_{p}^{N-3} \backslash \Lambda} \phi\left(x_{2}, \ldots, x_{N-2}\right) \prod_{i=2}^{N-2}\left|x_{i}\right|_{p}^{s_{1 i}}\left|1-x_{i}\right|_{p}^{s_{(N-1) i}} \prod_{2 \leq i<j \leq N-2}\left|x_{i}-x_{j}\right|_{p}^{s_{i j}} \prod_{i=2}^{N-2} d x_{i},
$$

for $\operatorname{Re}\left(s_{i j}\right)>0$ for any $i j$, is a multivariate Igusa local zeta function. In characteristic zero, a general theory for this type of local zeta functions was elaborated by Loeser in [36]. In particular, these local zeta functions admit analytic continuations as rational functions of the variables $p^{-s_{i j}}$.

We want to highlight that the convergence of the multivariate local zeta functions depends on the fact that the locally constant function $\phi$ has compact support. For example, we consider the following integral: 


$$
J(s)=\int_{\mathbb{Q}_{p}}|x|_{p}^{s} d x, s \in \mathbb{C} .
$$

If the integral $J\left(s_{0}\right)$ exists for some $s_{0} \in \mathbb{R}$, then the integrals

$$
J_{0}\left(s_{0}\right)=\int_{\mathbb{Z}_{p}}|x|_{p}^{s_{0}} d x \text { and } J_{1}\left(s_{0}\right)=\int_{\mathbb{Q}_{p} \backslash \mathbb{Z}_{p}}|x|_{p}^{s_{0}} d x
$$

exist. The first integral $J_{0}\left(s_{0}\right)=\frac{1-p^{-1}}{1-p^{-1-s_{0}}}$ for $s_{0}>-1$. For the second integral, we use that $|x|_{p}^{s_{0}}$ is locally integrable, and thus

$$
J_{1}\left(s_{0}\right)=\sum_{j=1}^{\infty} \int_{p^{-j} \mathbb{Z}_{p}^{\times}}|x|_{p}^{s_{0}} d x=\sum_{j=1}^{\infty} p^{j+j s_{0}} \int_{\mathbb{Z}_{p}^{\times}} d x=\left(1-p^{-1}\right) \sum_{j=1}^{\infty} p^{j\left(1+s_{0}\right)}<\infty
$$

if and only if $s_{0}<-1$. Then, integral $J(s)$ does not exist for any complex value $s$.

Theorem 1 in [8] establishes that the $p$-adic open string $N$-point zeta function is a holomorphic function in a certain domain of $\mathbb{C}^{D}$ and that it admits an analytic continuation to $\mathbb{C}^{D}$ (denoted as $Z^{(N)}(\boldsymbol{s})$ ) as a rational function in the variables $p^{-s_{i j}}, i, j \in\{1, \ldots, N-1\}$. Hence, in the theory of local zeta functions, the convergence and the existence of a meromorphic continuation as a rational function in the variables $p^{-s_{i j}}, i, j \in\{1, \ldots, N-1\}$ of integrals of type (20) is a new and remarkable result.

The $p$-adic open string $N$-point zeta functions $Z^{(N)}(s)$ are regularizations of the amplitudes $\boldsymbol{A}^{(N)}(\boldsymbol{k})$. More precisely, we define

$$
\boldsymbol{A}^{(N)}(\boldsymbol{k})=\left.\boldsymbol{Z}^{(N)}(\underline{\boldsymbol{s}})\right|_{s_{i j}=k_{i} \boldsymbol{k}_{j}} \text { with } i \in\{1, \ldots, N-1\}, j \in T \text { or } i, j \in T,
$$

where $T=\{2, \ldots, N-2\}$. By Theorem 1 in [8], $A^{(N)}(\boldsymbol{k})$ are well-defined rational functions of the variables $p^{-k_{i} k_{j}}, i, j \in\{1, \ldots, N-1\}$, which agree with integrals (87) when they converge.

In the following two sections, using the methods given in [8], we compute the $p$-adic open string amplitudes in the cases $N=4,5$.

\section{1. p-Adic Open String 4-Point Amplitude}

The 4-point string amplitude is given by

$$
A^{(4)}(\boldsymbol{k})=\int_{\mathbb{Q}_{p}}\left|x_{2}\right|_{p}^{k_{1} k_{2}}\left|1-x_{2}\right|_{p}^{k_{3} k_{2}} d x_{2}
$$

We attach to this amplitude the local zeta function:

$$
\mathbf{Z}^{(4)}(s)=\int_{\mathbb{Q}_{p}}\left|x_{2}\right|_{p}^{s_{12}}\left|1-x_{2}\right|_{p}^{s_{32}} d x_{2} .
$$

We now divide the integration domain $\left(\mathbb{Q}_{p}\right)$ into sectors. Given $I \subseteq T=\{2\}$, the attached sector is defined as

$$
\operatorname{Sect}(I)=\left\{x_{i} \in \mathbb{Q}_{p} ;\left|x_{i}\right|_{p} \leq 1 \Longleftrightarrow i \in I\right\} .
$$

Then, we have two sectors:

\begin{tabular}{ccc}
\hline $\mathbf{I}$ & $\mathbf{T} \backslash \mathbf{I}$ & $\operatorname{Sect}(\mathbf{I})$ \\
\hline$\{2\}$ & $\varnothing$ & $\mathbb{Z}_{p}$ \\
\hline$\varnothing$ & $\{2\}$ & $\mathbb{Q}_{p} \backslash \mathbb{Z}_{p}$, \\
\hline
\end{tabular}


and

$$
\mathbf{Z}^{(4)}(\boldsymbol{s})=\mathbf{Z}^{(4)}(\boldsymbol{s}, \varnothing)+\mathbf{Z}^{(4)}(\boldsymbol{s},\{2\})
$$

where

$$
\mathbf{Z}^{(4)}(s ;\{2\}):=\int_{\mathbb{Z}_{p}}\left|x_{2}\right|_{p}^{s_{12}}\left|1-x_{2}\right|_{p}^{s_{32}} d x_{2}
$$

and

$$
\mathbf{Z}^{(4)}(s ; \varnothing):=\int_{\mathbb{Q}_{p} \backslash \mathbb{Z}_{p}}\left|x_{2}\right|_{p}^{s_{12}}\left|1-x_{2}\right|_{p}^{s_{32}} d x_{2} .
$$

We now compute $\boldsymbol{Z}^{(4)}(\boldsymbol{s} ;\{2\})$. By using the Examples 2 and 3:

$$
\begin{aligned}
\boldsymbol{Z}^{(4)}(\boldsymbol{s} ;\{2\}) & =\int_{\mathbb{Z}_{p}}\left|x_{2}\right|_{p}^{s_{12}} d x_{2}+\int_{\mathbb{Z}_{p}^{\times}}\left|1-x_{2}\right|_{p}^{s_{42}} d x_{2} \\
& =1-2 p^{-1}+\frac{\left(1-p^{-1}\right) p^{-1-s_{12}}}{1-p^{-1-s_{12}}}+\frac{\left(1-p^{-1}\right) p^{-1-s_{32}}}{1-p^{-1-s_{32}}},
\end{aligned}
$$

for $\operatorname{Re}\left(s_{12}\right)>-1$ and $\operatorname{Re}\left(s_{32}\right)>-1$.

We now consider integral $Z^{(4)}(s ; \varnothing)$. By using the ultrametric inequality $\left|1-x_{2}\right|_{p}=\left|x_{2}\right|_{p}$ for $x_{2} \in \mathbb{Q}_{p} \backslash \mathbb{Z}_{p}$,

$$
\mathbf{Z}^{(4)}(\boldsymbol{s} ; \varnothing)=\int_{\mathbb{Q}_{p} \backslash \mathbb{Z}_{p}}\left|x_{2}\right|_{p}^{s_{12}+s_{32}} d x_{2}
$$

To compute this last integral we proceed as follows. For $l \in \mathbb{N} \backslash\{0\}$, we set

$$
\begin{gathered}
\left(\mathbb{Q}_{p} \backslash \mathbb{Z}_{p}\right)_{-l}:=\left\{x_{2} \in\left(\mathbb{Q}_{p} \backslash \mathbb{Z}_{p}\right) ;-l \leq \operatorname{ord}\left(x_{2}\right) \leq-1\right\}, \\
\left(p \mathbb{Z}_{p}\right)_{l}:=\left\{x_{2} \in\left(p \mathbb{Z}_{p}\right) ; 1 \leq \operatorname{ord}\left(x_{2}\right) \leq l\right\},
\end{gathered}
$$

and

$$
\boldsymbol{I}_{-l}(\boldsymbol{s}):=\int_{\left(\mathbb{Q}_{p} \backslash \mathbb{Z}_{p}\right)_{-l}}\left|x_{2}\right|_{p}^{s_{12}+s_{32}} d x_{2} .
$$

Notice that $\left(\mathbb{Q}_{p} \backslash \mathbb{Z}_{p}\right)_{-l^{\prime}}\left(p \mathbb{Z}_{p}\right)_{l}$ are compact sets and that

$$
\begin{aligned}
\left(\mathbb{Q}_{p} \backslash \mathbb{Z}_{p}\right)_{-l} & \rightarrow\left(p \mathbb{Z}_{p}\right)_{l} \\
x_{2} & \rightarrow \frac{1}{y_{2}},
\end{aligned}
$$

is an analytic change of variables satisfying $d x_{2}=\frac{d y_{2}}{\left|y_{2}\right|_{p}^{2}}$, then using this change of variables, we have

$$
I_{-l}(s)=\int_{\left(p \mathbb{Z}_{p}\right)_{l}} \frac{d y_{2}}{\left|y_{2}\right|_{p}^{s_{12}+s_{32}+2}} .
$$

Now, by using that $\int_{p \mathbb{Z}_{p}} \frac{1}{|y|_{p}^{s}} d y$ converges for $\operatorname{Re}(s)<1$ and the dominated convergence theorem, $\lim _{l \rightarrow \infty} I_{-l}(s)=Z^{(4)}(s ; \varnothing)$, i.e. 


$$
\mathbf{Z}^{(4)}(s ;\{\varnothing\})=\int_{p \mathbb{Z}_{p}} \frac{d y_{2}}{\left|y_{2}\right|_{p}^{s_{12}+s_{32}+2}}=\frac{\left(1-p^{-1}\right) p^{1+s_{12}+s_{32}}}{1-p^{1+s_{12}+s_{32}}},
$$

on $\operatorname{Re}\left(s_{12}\right)+\operatorname{Re}\left(s_{32}\right)<-1$. Therefore

$$
\mathbf{Z}^{(4)}(\boldsymbol{s})=\frac{p-2}{p}+\left(1-p^{-1}\right)\left\{\frac{p^{-1-s_{12}}}{1-p^{-1-s_{12}}}+\frac{p^{-1-s_{32}}}{1-p^{\left(-1-s_{32}\right)}}+\frac{p^{1+s_{12}+s_{32}}}{1-p^{1+s_{12}+s_{32}}}\right\},
$$

this function is holomorphic in

$$
\operatorname{Re}\left(s_{12}\right)>-1, \operatorname{Re}\left(s_{32}\right)>-1 \text { and } \operatorname{Re}\left(s_{12}\right)+\operatorname{Re}\left(s_{32}\right)<-1,
$$

which is a nonempty open subset in $\mathbb{C}^{2}$ because it contains the open set defined by the conditions:

$$
-\frac{2}{3}<\operatorname{Re}\left(s_{12}\right)<-\frac{1}{2} \text { and }-\frac{2}{3}<\operatorname{Re}\left(s_{32}\right)<-\frac{1}{2} .
$$

We denote the meromorphic continuation of integral $\boldsymbol{Z}^{(4)}(\boldsymbol{s})$ also by $\boldsymbol{Z}^{(4)}(\boldsymbol{s})$. Now, we regularize the $p$-adic open string 4-point amplitude using $Z^{(4)}(s)$ :

$$
\begin{aligned}
A^{(4)}(\boldsymbol{k}) & :=\left.Z^{(4)}(s)\right|_{s_{12}=k_{1} k_{2}, s_{32}=k_{3} k_{2}} \\
& =\frac{p-2}{p}+\left(1-p^{-1}\right)\left\{\frac{p^{-1-k_{1} k_{2}}}{1-p^{-1-k_{1} k_{2}}}+\frac{p^{-1-k_{2} k_{3}}}{1-p^{-1-k_{2} k_{3}}}+\frac{p^{1+k_{1} k_{2}+k_{2} k_{3}}}{1-p^{1+k_{1} k_{2}+k_{2} k_{3}}}\right\} \\
& =\frac{p-2}{p}+\left(1-p^{-1}\right)\left\{\frac{p^{-1-k_{1} k_{2}}}{1-p^{-1-k_{1} k_{2}}}+\frac{p^{-1-k_{2} k_{3}}}{1-p^{-1-k_{2} k_{3}}}+\frac{p^{-1-k_{2} k_{4}}}{1-p^{-1-k_{2} k_{4}}}\right\}
\end{aligned}
$$

This amplitude can be rewritten as

$$
\boldsymbol{A}^{(4)}(\boldsymbol{k})=K_{4}+\frac{1}{2} \sum_{i<j} x_{i j}
$$

with

$$
x_{i j}:=\frac{p-1}{p} \frac{p^{-1-k_{i} k_{j}}}{1-p^{-1-k_{i} k_{j}}}
$$

where $\sum_{i<j}$ means the summation over all pairs of compatible channels $i j$. Then

$$
\boldsymbol{A}^{(4)}(\boldsymbol{k})=K_{4}+\frac{1}{2}\left\{x_{12}+x_{13}+x_{14}+x_{23}+x_{24}+x_{34}\right\} \text {. }
$$

Indeed, by using that $\sum_{i=1}^{4} \boldsymbol{k}_{i}=0, \boldsymbol{k}_{i}^{2}=2$, we obtain

$$
\begin{aligned}
& k_{1} k_{3}+k_{2} k_{3}+k_{3} k_{4}+2=0 \\
& k_{1} k_{2}+k_{1} k_{3}+k_{1} k_{4}+2=0 \\
& k_{1} k_{2}+k_{2} k_{3}+k_{2} k_{4}+2=0 \\
& k_{1} k_{4}+k_{2} k_{4}+k_{3} k_{4}+2=0
\end{aligned}
$$

From (23)-(26), we obtain

$$
k_{1} k_{2}=k_{3} k_{4}, k_{1} k_{3}=k_{2} k_{4}, k_{1} k_{4}=k_{2} k_{3} .
$$

Then

$$
\boldsymbol{A}^{(4)}(\boldsymbol{k})=K_{4}+x_{12}+x_{23}+x_{24}
$$


which agrees with (22).

\section{2. $p$-Adic Open String 5-Point Amplitude}

The $p$-adic open string 5-point amplitude is given by

$$
A^{(5)}(k)=\int_{\mathbb{Q}_{p}^{2}}\left|x_{2}\right|_{p}^{k_{1} k_{2}}\left|x_{3}\right|_{p}^{k_{1} k_{3}}\left|1-x_{2}\right|_{p}^{k_{4} k_{2}}\left|1-x_{3}\right|_{p}^{k_{4} k_{3}}\left|x_{2}-x_{3}\right|_{p}^{k_{2} k_{3}} d x_{2} d x,
$$

and the 5-point local zeta function is given by

$$
\mathbf{Z}^{(5)}(s)=\int_{\mathbb{Q}_{p}^{2}}\left|x_{2}\right|_{p}^{s_{12}}\left|x_{3}\right|_{p}^{s_{13}}\left|1-x_{2}\right|_{p}^{s_{42}}\left|1-x_{3}\right|_{p}^{s_{43}}\left|x_{2}-x_{3}\right|_{p}^{s_{23}} d x_{2} d x_{3},
$$

where $s_{i j} \in \mathbb{C}$. We divide the integration domain $\mathbb{Q}_{p}^{2}$ in sectors. Given $I \subseteq T=\{2,3\}$ the attached sector is defined as

$$
\operatorname{Sect}(I)=\left\{\left(x_{1}, x_{2}\right) \in \mathbb{Q}_{p}^{2} ;\left|x_{i}\right|_{p} \leq 1 \Longleftrightarrow i \in I\right\} .
$$

Table 1 shows all the sectors for the computation of the 5-point amplitude.

Table 1. Sectors of the 5-point amplitude.

\begin{tabular}{ccc}
\hline $\mathbf{I}$ & $\mathbf{T} \backslash \mathbf{I}$ & $\mathbf{S e c t}(\mathbf{I})$ \\
\hline$\varnothing$ & $\{2,3\}$ & $\left(\mathbb{Q}_{p} \backslash \mathbb{Z}_{p}\right) \times\left(\mathbb{Q}_{p} \backslash \mathbb{Z}_{p}\right)$ \\
\hline$\{2\}$ & $\{3\}$ & $\mathbb{Z}_{p} \times\left(\mathbb{Q}_{p} \backslash \mathbb{Z}_{p}\right)$ \\
\hline$\{3\}$ & $\{2\}$ & $\left(\mathbb{Q}_{p} \backslash \mathbb{Z}_{p}\right) \times \mathbb{Z}_{p}$ \\
\hline$\{2,3\}$ & $\varnothing$ & $\mathbb{Z}_{p} \times \mathbb{Z}_{p}$. \\
\hline
\end{tabular}

Now, by using the notation

$$
Z^{(5)}(s ; I)=\int_{\operatorname{Sect}(I)}\left|x_{2}\right|_{p}^{s_{12}}\left|x_{3}\right|_{p}^{s_{13}}\left|1-x_{2}\right|_{p}^{s_{42}}\left|1-x_{3}\right|_{p}^{s_{43}}\left|x_{2}-x_{3}\right|_{p}^{s_{23}} d x_{2} d x_{3}
$$

we have

$$
\boldsymbol{Z}^{(5)}(s)=\mathbf{Z}^{(5)}(\boldsymbol{s} ; \varnothing)+\boldsymbol{Z}^{(5)}(\boldsymbol{s} ;\{2\})+\mathbf{Z}^{(5)}(\boldsymbol{s} ;\{3\})+\mathbf{Z}^{(5)}(\boldsymbol{s} ;\{2,3\}) .
$$

Case 1. $\operatorname{Sect}(\{\varnothing\})=\left(\mathbb{Q}_{p} \backslash \mathbb{Z}_{p}\right) \times\left(\mathbb{Q}_{p} \backslash \mathbb{Z}_{p}\right)$.

By changing of variables as $x_{2} \rightarrow \frac{1}{u}, x_{3} \rightarrow \frac{1}{v}$ and using Lemma 7 of [8], we have

$$
\begin{aligned}
& \boldsymbol{Z}^{(5)}(s ;\{\varnothing\})=\int_{\left(\mathbb{Q}_{p} \backslash \mathbb{Z}_{p}\right)^{2}}\left|x_{2}\right|_{p}^{s_{12}+s_{42}}\left|x_{3}\right|_{p}^{s_{13}+s_{43}}\left|x_{2}-x_{3}\right|_{p}^{s_{23}} d x_{2} d x_{3} . \\
& =\int_{\left(p \mathbb{Z}_{p}\right)^{2}}|u|_{p}^{-s_{12}-s_{42}-2}|v|_{p}^{-s_{13}-s_{43}-2}\left|\frac{1}{u}-\frac{1}{v}\right|_{p}^{s_{23}} d u d v \\
& =p^{-2+s_{12}+s_{42}+s_{23}+2+s_{13}+s_{43}+s_{23}+2-s_{23}} \int_{\mathbb{Z}_{p}^{2}} \frac{|v-u|_{p}^{s_{23}}}{|u|_{p}^{s_{12}+s_{42}+s_{23}+2}|v|_{p}^{s_{13}+s_{43}+s_{23}+2}} d u d v \\
& =\frac{p^{2+s_{12}+s_{42}+s_{23}+s_{13}+s_{43}}\left(1-p^{-1}\right)}{1-p^{2+s_{12}+s_{42}+s_{23}+s_{13}+s_{43}}}\left[\frac{\left(1-p^{-1}\right) p^{1+s_{12}+s_{42}+s_{23}}}{1-p^{1+s_{12}+s_{42}+s_{23}}}\right. \\
& \left.+\frac{\left(1-p^{-1}\right) p^{1+s_{13}+s_{43}+s_{23}}}{1-p^{1+s_{13}+s_{43}+s_{23}}}+\frac{\left(1-p^{-1}\right) p^{-1-s_{23}}}{1-p^{-1-s_{23}}}+(p-2) p^{-1}\right] \text {. }
\end{aligned}
$$

The integral $\mathbf{Z}^{(5)}(\boldsymbol{s} ;\{\varnothing\})$ is holomorphic in the solution set of the following conditions: 


$$
\begin{gathered}
\operatorname{Re}\left(s_{12}\right)+\operatorname{Re}\left(s_{42}\right)+\operatorname{Re}\left(s_{23}\right)<-1 \\
\operatorname{Re}\left(s_{13}\right)+\operatorname{Re}\left(s_{43}\right)+\operatorname{Re}\left(s_{23}\right)<-1 \\
\\
\operatorname{Re}\left(s_{23}\right)>-1 \\
\operatorname{Re}\left(s_{12}\right)+\operatorname{Re}\left(s_{42}\right)+\operatorname{Re}\left(s_{13}\right)+\operatorname{Re}\left(s_{43}\right)+\operatorname{Re}\left(s_{23}\right)<-2 .
\end{gathered}
$$

Case 2. $\operatorname{Sect}(\{2\})=\mathbb{Z}_{p} \times\left(\mathbb{Q}_{p} \backslash \mathbb{Z}_{p}\right)$.

In this case, $\left|x_{2}\right|_{p} \leq 1$ and $\left|x_{3}\right|_{p}>1$, now by ultrametric property $\left|1-x_{3}\right|_{p}=\left|x_{3}\right|_{p}$ and $\left|x_{2}-x_{3}\right|_{p}=\left|x_{3}\right|_{p}$, then

$$
\begin{aligned}
Z^{(5)}(s ;\{2\}) & =\int_{\mathbb{Z}_{p} \times\left(\mathbb{Q}_{p} \backslash \mathbb{Z}_{p}\right)}\left|x_{2}\right|_{p}^{s_{12}}\left|x_{3}\right|_{p}^{s_{13}}\left|1-x_{2}\right|_{p}^{s_{42}}\left|1-x_{3}\right|_{p}^{s_{43}}\left|x_{2}-x_{3}\right|_{p}^{s_{23}} d x_{2} d x_{3} \\
& =\int_{\mathbb{Z}_{p} \times\left(\mathbb{Q}_{p} \backslash \mathbb{Z}_{p}\right)}\left|x_{2}\right|_{p}^{s_{12}}\left|x_{3}\right|_{p}^{s_{13}+s_{43}+s_{23}}\left|1-x_{2}\right|_{p}^{s_{42}} d x_{2} d x_{3} \\
& =\int_{\mathbb{Z}_{p}}\left|x_{2}\right|_{p}^{s_{12}}\left|1-x_{2}\right|_{p}^{s_{42}} d x_{2} \int_{\left(\mathbb{Q}_{p} \backslash \mathbb{Z}_{p}\right)}\left|x_{3}\right|_{p}^{s_{13}+s_{43}+s_{23}} d x_{3} \\
& =: \mathbb{Z}_{0}^{(5)}(s ;\{2\}) \mathbf{Z}_{1}^{(5)}(s ;\{2\}) .
\end{aligned}
$$

The calculation of $\boldsymbol{Z}^{(5)}(\boldsymbol{s} ;\{2\}, 0)$ is similar to the case of integral (21):

$$
\mathbf{Z}^{(5)}(\boldsymbol{s} ;\{2\}, 0)=p^{-1}(p-2)+\frac{\left(1-p^{-1}\right) p^{-1-s_{12}}}{1-p^{-1-s_{12}}}+\frac{\left(1-p^{-1}\right) p^{-1-s_{42}}}{1-p^{-1-s_{42}}} .
$$

The integral $Z^{(5)}(s ;\{2\}, 0)$ is holomorphic in the domain

$$
\operatorname{Re}\left(s_{12}\right)>-1 \text { and } \operatorname{Re}\left(s_{42}\right)>-1 .
$$

The calculation of $\boldsymbol{Z}^{(5)}(s ;\{2\}, 1)$ is similar to the calculation of $\mathbf{Z}^{(4)}(\boldsymbol{s} ;\{\varnothing\})$ given in the Subsection of 4-point zeta function. By changing of variables as $x_{3}=\frac{1}{y_{3}}, d x_{3}=\frac{d y_{3}}{\left|y_{3}\right|_{p}^{2}}$, we obtain

$$
\begin{aligned}
& \boldsymbol{Z}^{(5)}(\boldsymbol{s} ;\{2\}, 1)=\int_{\mathbb{Q}_{p} \backslash \mathbb{Z}_{p}}\left|x_{3}\right|_{p}^{s_{13}+s_{43}+s_{23}} d x_{3}=\int_{p \mathbb{Z}_{p}} \frac{d y_{3}}{\left|y_{3}\right|_{p}^{2+s_{13}+s_{43}+s_{23}}} \\
& =\frac{\left(1-p^{-1}\right) p^{1+s_{13}+s_{43}+s_{23}}}{1-p^{1+s_{13}+s_{43}+s_{23}}} .
\end{aligned}
$$

The integral $Z^{(5)}(s ;\{2\}, 1)$ is holomorphic in

$$
\operatorname{Re}\left(s_{13}\right)+\operatorname{Re}\left(s_{43}\right)+\operatorname{Re}\left(s_{23}\right)<-1 .
$$

Case 3. $\operatorname{Sect}(\{3\})=\left(\mathbb{Q}_{p} \backslash \mathbb{Z}_{p}\right) \times \mathbb{Z}_{p}$.

In this case, $\left|x_{2}\right|_{p}>1$, by the ultrametric inequality $\left|1-x_{2}\right|_{p}=\left|x_{2}\right|_{p}$ and $\left|x_{2}-x_{3}\right|_{p}=$ $\left|x_{2}\right|_{p}$, then 


$$
\begin{aligned}
Z^{(5)}(s ;\{3\}) & =\int_{\left(\mathbb{Q}_{p} \backslash \mathbb{Z}_{p}\right) \times \mathbb{Z}_{p}}\left|x_{2}\right|_{p}^{s_{12}}\left|x_{3}\right|_{p}^{s_{13}}\left|1-x_{2}\right|_{p}^{s_{42}}\left|1-x_{3}\right|_{p}^{s_{43}}\left|x_{2}-x_{3}\right|_{p}^{s_{23}} d x_{2} d x_{3} \\
& =\int_{\mathbb{Q}_{p} \backslash \mathbb{Z}_{p} \times \mathbb{Z}_{p}}\left|x_{2}\right|_{p}^{s_{12}+s_{42}+s_{23}}\left|x_{3}\right|_{p}^{s_{13}}\left|1-x_{3}\right|_{p}^{s_{43}} d x_{2} d x_{3} \\
& =\int_{\mathbb{Z}_{p}}\left|x_{3}\right|_{p}^{s_{13}}\left|1-x_{3}\right|_{p}^{s_{43}} d x_{3} \int_{\mathbb{Q}_{p} \backslash \mathbb{Z}_{p}}\left|x_{2}\right|_{p}^{s_{12}+s_{42}+s_{23}} d x_{2} \\
& =: \mathbb{Z}_{0}^{(N)}(s ;\{3\}) \mathbf{Z}_{1}^{(N)}(s ;\{3\}) .
\end{aligned}
$$

These integrals are similar to the ones obtained in the case of $\operatorname{Sect}(\{2\})$ :

$$
\mathbf{Z}^{(5)}(\underline{s} ;\{3\}, 0)=p^{-1}(p-2)+\frac{\left(1-p^{-1}\right) p^{-1-s_{13}}}{1-p^{-1-s_{13}}}+\frac{\left(1-p^{-1}\right) p^{-1-s_{43}}}{1-p^{-1-s_{43}}},
$$

the integral $Z^{(5)}(s ;\{3\}, 0)$ is holomorphic in

$$
\operatorname{Re}\left(s_{13}\right)>-1 \text { and } \operatorname{Re}\left(s_{43}\right)>-1 .
$$

And

$$
\mathbf{Z}^{(5)}(s ;\{3\}, 1)=\frac{\left(1-p^{-1}\right) p^{1+s_{12}+s_{42}+s_{23}}}{1-p^{1+s_{12}+s_{42}+s_{23}}},
$$

the integral $Z^{(5)}(s ;\{3\}, 1)$ is holomorphic in

$$
\operatorname{Re}\left(s_{12}\right)+\operatorname{Re}\left(s_{42}\right)+\operatorname{Re}\left(s_{23}\right)<-1 .
$$

Case 4. $\operatorname{Sect}(\{2,3\})=\mathbb{Z}_{p} \times \mathbb{Z}_{p}$. we have

Using the partition $\mathbb{Z}_{p}^{2}=\left(p \mathbb{Z}_{p}\right)^{2} \sqcup S_{0}^{2}$, with $S_{0}^{2}=p \mathbb{Z}_{p} \times \mathbb{Z}_{p}^{\times} \sqcup \mathbb{Z}_{p}^{\times} \times p \mathbb{Z}_{p} \sqcup \mathbb{Z}_{p}^{\times} \times \mathbb{Z}_{p}^{\times}$,

$$
\begin{aligned}
Z^{(5)}(s ;\{2,3\}) & =\int_{\mathbb{Z}_{p} \times \mathbb{Z}_{p}}\left|x_{2}\right|_{p}^{s_{12}}\left|x_{3}\right|_{p}^{s_{13}}\left|1-x_{2}\right|_{p}^{s_{42}}\left|1-x_{3}\right|_{p}^{s_{43}}\left|x_{2}-x_{3}\right|_{p}^{s_{23}} d x_{2} d x_{3} \\
& =: I_{31}(s)+I_{32}(s)+I_{33}(s)+I_{34}(s),
\end{aligned}
$$

where

$$
\begin{aligned}
& I_{31}(s):=\int_{\left(p \mathbb{Z}_{p}\right)^{2}}\left|x_{2}\right|_{p}^{s_{12}}\left|x_{3}\right|_{p}^{s_{13}}\left|x_{2}-x_{3}\right|_{p}^{s_{23}} d x_{2} d x_{3}, \\
& I_{32}(s):=\int_{p \mathbb{Z}_{p} \times \mathbb{Z}_{p}^{\times}}\left|x_{2}\right|_{p}^{s_{12}}\left|1-x_{3}\right|_{p}^{s_{43}} d x_{2} d x_{3}, \\
& I_{33}(s):=\int_{\mathbb{Z}_{p}^{\times} \times p \mathbb{Z}_{p}}\left|x_{3}\right|_{p}^{s_{13}}\left|1-x_{2}\right|_{p}^{s_{42}} d x_{2} d x_{3}, \\
& I_{34}(s):=\int_{\mathbb{Z}_{p}^{\times} \times \mathbb{Z}_{p}^{\times}}\left|1-x_{2}\right|_{p}^{s_{42}}\left|1-x_{3}\right|_{p}^{s_{43}}\left|x_{2}-x_{3}\right|_{p}^{s_{23}} d x_{2} d x_{3} .
\end{aligned}
$$

Using Lemma 4 of [8], we have

$$
\begin{aligned}
I_{31}(\underline{s}) & =\frac{p^{-2-s_{12}-s_{13}-s_{23}}}{1-p^{-2-s_{12}-s_{13}-s_{23}}}\left[\frac{p^{-1-s_{12}}\left(1-p^{-1}\right)^{2}}{1-p^{-1-s_{12}}}+\frac{p^{-1-s_{13}}\left(1-p^{-1}\right)^{2}}{1-p^{-1-s_{13}}}\right. \\
& \left.+\frac{p^{-1-s_{23}}\left(1-p^{-1}\right)^{2}}{1-p^{-1-s_{23}}}+(p-1)(p-2) p^{-2}\right] .
\end{aligned}
$$


The integral $I_{31}(\underline{s})$ is holomorphic in

$$
\operatorname{Re}\left(s_{12}\right)>-1, \operatorname{Re}\left(s_{13}\right)>-1, \operatorname{Re}\left(s_{23}\right)>-1, \text { and } \operatorname{Re}\left(s_{12}\right)+\operatorname{Re}\left(s_{13}\right)+\operatorname{Re}\left(s_{23}\right)>-2 .
$$

Using Examples 2 and 3,

$$
I_{32}(s)=\frac{\left(1-p^{-1}\right) p^{-1-s_{12}}}{1-p^{-1-s_{12}}}\left[\frac{\left(1-p^{-1}\right) p^{-1-s_{43}}}{1-p^{-1-s_{43}}}+p^{-1}(p-2)\right],
$$

and

$$
I_{33}(s)=\frac{\left(1-p^{-1}\right) p^{-1-s_{13}}}{1-p^{-1-s_{13}}}\left[\frac{\left(1-p^{-1}\right) p^{-1-s_{42}}}{1-p^{-1-s_{42}}}+p^{-1}(p-2)\right] .
$$

These integrals are holomorphic in

$$
\operatorname{Re}\left(s_{12}\right)>-1, \operatorname{Re}\left(s_{13}\right)>-1, \operatorname{Re}\left(s_{42}\right)>-1, \text { and } \operatorname{Re}\left(s_{43}\right)>-1 \text {. }
$$

Finally, by Example 6,

$$
\begin{aligned}
I_{34}(s) & =p^{-2}(p-2)(p-3)+(p-2) \frac{p^{-2-s_{23}}\left(1-p^{-1}\right)}{1-p^{-1-s_{23}}} \\
& +(p-2) \frac{p^{-2-s_{42}}\left(1-p^{-1}\right)}{1-p^{-1-s_{42}}}+(p-2) \frac{p^{-2-s_{43}}\left(1-p^{-1}\right)}{1-p^{-1-s_{43}}} .
\end{aligned}
$$

This integral is holomorphic in

$$
\operatorname{Re}\left(s_{42}\right)>-1, \operatorname{Re}\left(s_{43}\right)>-1, \text { and } \operatorname{Re}\left(s_{23}\right)>-1 \text {. }
$$

In conclusion, the 5-point local zeta functions is holomorphic on the region of $\mathbb{C}^{5}$ defined by

$$
\begin{aligned}
\operatorname{Re}\left(s_{12}\right)>-1, & \operatorname{Re}\left(s_{13}\right)>-1, \operatorname{Re}\left(s_{42}\right)>-1, \text { and } \operatorname{Re}\left(s_{43}\right)>-1, \\
& \operatorname{Re}\left(s_{12}\right)+\operatorname{Re}\left(s_{42}\right)+\operatorname{Re}\left(s_{23}\right)<-1, \\
& \operatorname{Re}\left(s_{13}\right)+\operatorname{Re}\left(s_{43}\right)+\operatorname{Re}\left(s_{23}\right)<-1, \\
& \operatorname{Re}\left(s_{12}\right)+\operatorname{Re}\left(s_{13}\right)+\operatorname{Re}\left(s_{23}\right)>-2 . \\
\operatorname{Re}\left(s_{12}\right)+ & \operatorname{Re}\left(s_{42}\right)+\operatorname{Re}\left(s_{13}\right)+\operatorname{Re}\left(s_{43}\right)+\operatorname{Re}\left(s_{23}\right)<-2 .
\end{aligned}
$$

which is a nonempty subset, since it contains the open set

$$
\begin{gathered}
-\frac{2}{3}<\operatorname{Re}\left(s_{i j}\right)<0 \\
-\frac{2}{3}<\operatorname{Re}\left(s_{1 i}\right)<-\frac{1}{2}, \\
-\frac{2}{3}<\operatorname{Re}\left(s_{(N-1) i}\right)<-\frac{1}{2} .
\end{gathered}
$$

We regularize the $p$-adic open string 5-point amplitude using the meromorphic continuation of $\boldsymbol{Z}^{(5)}(\boldsymbol{s})$ by setting

$$
A^{(5)}(\boldsymbol{k}):=\left.Z^{(5)}(s)\right|_{s_{12}=k_{1} k_{2}, s_{13}=k_{1} k_{3}, s_{42}=k_{4} k_{2}, s_{43}=k_{4} k_{3}, s_{23}=k_{2} k_{3}} .
$$

The amplitude $\boldsymbol{A}^{(5)}(\boldsymbol{k})$ agrees with the one computed in [14] using the Feynman rules of the effective Lagrangian:

$$
\boldsymbol{A}^{(5)}(\boldsymbol{k})=K_{5}+K_{4} \sum_{i<j} x_{i j}+\sum_{\substack{\{i, j, k, l\} \subset\{1,2,3,4,5\} \\ i<j, k<l}} x_{i j} x_{k l},
$$


where $K_{5}=\frac{(p-2)(p-3)}{p^{2}}, K_{4}=\frac{p-2}{p}$, and $i j$ and $k l$ are summed over all pairs of compatible channels. By a pair of compatible channels $i j, k l$ we mean that $i, j, k$ and $l$ are different, and that $i<j, k<l$.

\section{3. $p$-Adic Open String N-Point Amplitudes}

We fix an integer $N \geq 4$ and consider the general $N$-point zeta function:

$$
\mathbf{Z}^{(N)}(\boldsymbol{s})=\int_{\mathbb{Q}_{p}^{N-3}} \prod_{i=2}^{N-2}\left|x_{i}\right|_{p}^{s_{1 i}}\left|1-x_{i}\right|_{p}^{s_{(N-1) i}} \prod_{2 \leq i<j \leq N-2}\left|x_{i}-x_{j}\right|_{p}^{s_{i j}} \prod_{i=2}^{N-2} d x_{i} .
$$

We divide the domain of integration $\mathbb{Q}_{p}^{N-3}$ into sectors. Given $I \subseteq T=\{2,3, \ldots, N-2\}$ the attached sector is defined as

$$
\operatorname{Sect}(I)=\left\{\left(x_{2}, \ldots, x_{N-2}\right) \in \mathbb{Q}_{p}^{N-3} ;\left|x_{i}\right|_{p} \leq 1 \Leftrightarrow i \in I\right\} .
$$

Hence, the $N$-point zeta function (40) can be written as

$$
\mathbf{Z}^{(N)}(s)=\sum_{I \subseteq T} Z^{(N)}(s ; I),
$$

where

$$
\boldsymbol{Z}^{(N)}(s ; I):=\int_{\operatorname{Sect}(I)} F(\boldsymbol{s}, \boldsymbol{x} ; N) \prod_{i=2}^{N-2} d x_{i}
$$

with

$$
F(s, x ; N):=\prod_{i=2}^{N-2}\left|x_{i}\right|_{p}^{s_{1 i}}\left|1-x_{i}\right|_{p}^{s_{(N-1) i}} \prod_{2 \leq i<j \leq N-2}\left|x_{i}-x_{j}\right|_{p}^{s_{i j}}
$$

and $x=\left(x_{2}, \ldots, x_{N-2}\right) \in \mathbb{Q}_{p}^{N-3}$.

By Lemma 2 in [8],

$$
\begin{aligned}
& Z^{(N)}(s ; I)=p^{M(s)}\left\{\int_{\mathbb{Z}_{p}^{I I !}} \prod_{i \in I}\left|x_{i}\right|_{p}^{s_{1 i}}\left|1-x_{i}\right|_{p}^{s_{(N-1) i}} \prod_{\substack{2 \leq i<j \leq N-2 \\
i, j \in I}}\left|x_{i}-x_{j}\right|_{p}^{s_{i j}} \prod_{i \in I} d x_{i}\right\} \\
& \times\left\{\int_{\mathbb{Z}_{p}^{T \backslash I \mid}} \frac{\prod_{\substack{2 \leq i<j \leq N-2 \\
i, j \in T \backslash I}}\left|x_{i}-x_{j}\right|_{p}^{s_{i j}}}{\prod_{i \in T \backslash I}\left|x_{i}\right|_{p}^{2+s_{1 i}+s_{(N-1) i}+\sum_{2 \leq j \leq N-2, j \neq i} s_{i j}}} \prod_{i \in T \backslash I} d x_{i}\right\} \\
& =: p^{M(s)} Z_{0}^{(N)}(s ; I) Z_{1}^{(N)}(s ; T \backslash I),
\end{aligned}
$$

where

$$
M(s):=|T \backslash I|+\sum_{i \in T \backslash I}\left(s_{1 i}+s_{(N-1) i}\right)+\sum_{\substack{2 \leq i<j \leq N-2 \\ i \in T \backslash I, j \in T}} s_{i j}+\sum_{\substack{2 \leq i<j \leq N-2 \\ i \in I, j \in T \backslash I}} s_{i j} .
$$

In addition $Z_{0}^{(N)}(s ; I), Z_{1}^{(N)}(s ; T \backslash I)$ are multivariate local zeta functions and

$$
Z^{(N)}(s)=\sum_{I \subseteq T} p^{M(s)} Z_{0}^{(N)}(s ; I) Z_{1}^{(N)}(s ; T \backslash I),
$$


with the convention that $Z_{i}^{(N)}(s ; \varnothing)=1$ for $i=0,1$.

In [8] we show that $Z^{(N)}(s)$ has an analytic continuation to the whole $\mathbb{C}^{D}$ as a rational function in the variables $p^{-s_{i j}}$ by showing that all functions that appear on the right-hand side of $(41)$ are holomorphic in a region $H(\mathbb{C})$ in $\mathbb{C}^{D}$ (Definition 3 and Remarks 9 and 10 in [8]) defined by

$$
\begin{gathered}
|J|+\sum_{i \in J}\left(\operatorname{Re}\left(s_{1 i}\right)+\operatorname{Re}\left(s_{(N-1) i}\right)\right)+\sum_{\substack{2 \leq i<j \leq N-2 \\
i \in J}} \operatorname{Re}\left(s_{i j}\right) \\
+\sum_{\substack{2 \leq i<j \leq N-2 \\
i \in T \backslash J, j \in J}} \operatorname{Re}\left(s_{i j}\right)<0 \text { for } J \in \mathfrak{F}_{1} ; \\
|K|-1+\sum_{\substack{2 \leq i<j \leq N-2 \\
i, j \in K}} \operatorname{Re}\left(s_{i j}\right)>0 \text { for } K \in \mathfrak{F}_{2} ; \\
1+\operatorname{Re}\left(s_{i j}\right)>0 \text { for } i j \in \mathcal{G} \subseteq\{i j ; 2 \leq i<j \leq N-2\},
\end{gathered}
$$

where $\mathfrak{F}_{1}, \mathfrak{F}_{2}$ are families of nonempty subsets of $T$, and $\mathcal{G}$ is a nonempty subset of $\{i j ; 2 \leq i<j \leq N-2, i, j \in T\}$.

$$
|J|+\sum_{i \in S} \operatorname{Re}\left(s_{t i}\right)+\sum_{2 \leq i<j \leq N-2, i, j \in J} \operatorname{Re}\left(s_{i j}\right)>0 \text { for } J \times S \in \mathfrak{F}_{3},
$$

with $S \subseteq J, t \in\{1, N-1\}$, and $\mathfrak{F}_{3}$ a family of nonempty subsets of $I \times I$;

$$
|K|-1+\sum_{\substack{2 \leq i<j \leq N-2 \\ i, j \in K}} \operatorname{Re}\left(s_{i j}\right)>0 \text { for } K \in \mathfrak{F}_{4},
$$

where $\mathfrak{F}_{4}$ 's a family of nonempty subsets of $I$;

$$
1+\operatorname{Re}\left(s_{i j}\right)>0 \text { for } i j \in G_{T},
$$

where $G_{T}$ is a nonempty subset of $\{2 \leq i<j \leq N-2, i, j \in J\}$ with $(N-1) i, 1 i \in G_{T}$.

In Lemma 9 of [8], we show that region $H(\mathbb{C})$ contains and open and connected subset of $\mathbb{C}^{D}$ defined by the conditions

$$
\begin{gathered}
-\frac{2}{3 N_{1}}<\operatorname{Re}\left(s_{i j}\right)<0, \\
-\frac{2}{3}<\operatorname{Re}\left(s_{1 i}\right)<-\frac{1}{2}, \\
-\frac{2}{3}<\operatorname{Re}\left(s_{(N-1) i}\right)<-\frac{1}{2},
\end{gathered}
$$

for $N \geqslant 5, N_{1}=\frac{(N-4)(N-3)}{2}, i, j \in\{2, \ldots, N-2\}$. For the case $N=4$ we only consider conditions (49) and (50). Like in the cases $N=4$ and $N=5$, the key point is to reduce the integrals $Z_{0}^{(N)}(s ; I), Z_{1}^{(N)}(s ; T \backslash I)$ to certain simple integrals, for which admit meromorphic continuations to the whole $\mathbb{C}^{D}$ as rational functions in the variables $p^{-s_{i j}}$.

We now state the meromorphic continuation of the open string $N$-point zeta function.

Theorem 1 (Theorem 1 in [8]). (1) The p-adic open string $N$-point zeta function, $Z^{(N)}(s)$, gives rise to a holomorphic function on $H(\mathbb{C})$, which contains an open and connected subset of $\mathbb{C}^{D}$. Furthermore, $Z^{(N)}(s)$ admits an analytic continuation to $\mathbb{C}^{D}$, denoted also as $Z^{(N)}(s)$, as a rational function in the variables $p^{-s_{i j}}, i, j \in\{1, \ldots, N-1\}$. The real parts of the poles of $Z^{(N)}(s)$ belong to a finite union of hyperplanes, the equations of these hyperplanes have the form (42)-(47) with the symbols ' $<$,' ' $>$ ' replaced by ' $=$ '. (2) If $s=\left(s_{i j}\right) \in \mathbb{C}^{D}$, with $\operatorname{Re}\left(s_{i j}\right) \geq 0$ for $i, j \in\{1, \ldots, N-1\}$, then the integral $Z^{(N)}(s)$ diverges to $+\infty$. 


\section{String Amplitudes over Non-Archimedean Local Fields}

\subsection{Non-Archimedean Local Fields}

A non-Archimedean local field $\mathbb{K}$ is a locally compact topological field with respect to a non-discrete topology, which comes from a norm $|\cdot|_{\mathbb{K}}$ satisfying

$$
|x+y|_{\mathbb{K}} \leq \max \left\{|x|_{\mathbb{K}},|y|_{\mathbb{K}}\right\},
$$

for $x, y \in \mathbb{K}$. A such norm is called an ultranorm or non-Archimedean. Any non-Archimedean local field $\mathbb{K}$ of characteristic zero is isomorphic (as a topological field) to a finite extension of $\mathbb{Q}_{p}$. The field $\mathbb{Q}_{p}$ is the basic example of non-Archimedean local field of characteristic zero. In the case of positive characteristic, $\mathbb{K}$ is isomorphic to a finite extension of the field of formal Laurent series $\mathbb{F}_{q}((T))$ over a finite field $\mathbb{F}_{q}$, where $q$ is a power of a prime number $p$.

The ring of integers of $\mathbb{K}$ is defined as

$$
R_{\mathbb{K}}=\left\{x \in \mathbb{K} ;|x|_{\mathbb{K}} \leq 1\right\} .
$$

Geometrically $R_{\mathbb{K}}$ is the unit ball of the normed space $\left(\mathbb{K},|\cdot|_{\mathbb{K}}\right)$. This ring is a domain of principal ideals with a unique maximal ideal, which is given by

$$
P_{\mathbb{K}}=\left\{x \in \mathbb{K} ;|x|_{\mathbb{K}}<1\right\} .
$$

We fix a generator $\pi$ of $P_{\mathbb{K}}$ i.e., $P_{\mathbb{K}}=\pi R_{\mathbb{K}}$. A such generator is also called a local uniformizing parameter of $\mathbb{K}$, and it plays the same role as $p$ in $\mathbb{Q}_{p}$.

The group of units of $R_{\mathbb{K}}$ is defined as

$$
R_{\mathbb{K}}^{\times}=\left\{x \in R_{\mathbb{K}} ;|x|_{\mathbb{K}}=1\right\} .
$$

The natural map $R_{\mathbb{K}} \rightarrow R_{\mathbb{K}} / P_{\mathbb{K}} \cong \mathbb{F}_{q}$ is called the reduction $\bmod P_{\mathbb{K}}$. The quotient $R_{\mathbb{K}} / P_{\mathbb{K}} \cong \mathbb{F}_{q}, q=p^{f}$, is called the residue field of $\mathbb{K}$. Every nonzero element $x$ of $\mathbb{K}$ can be written uniquely as $x=\pi^{\operatorname{ord}(x)} u, u \in R_{\mathbb{K}}^{\times}$. We set $\operatorname{ord}(0)=\infty$. The normalized valuation of $\mathbb{K}$ is the mapping

$$
\begin{aligned}
\mathbb{K} & \rightarrow \mathbb{Z} \cup\{\infty\} \\
x & \rightarrow \operatorname{ord}(x) .
\end{aligned}
$$

Then $|x|_{\mathbb{K}}=q^{-\operatorname{ord}(x)}$ and $|\pi|_{\mathbb{K}}=q^{-1}$.

We fix $\mathfrak{S} \subset R_{\mathbb{K}}$ a set of representatives of $\mathbb{F}_{q}$ in $R_{\mathbb{K}}$, i.e., $\mathfrak{S}$ is a set which is mapped bijectively onto $\mathbb{F}_{q}$ by the reduction $\bmod P_{\mathbb{K}}$. We assume that $0 \in \mathfrak{S}$. Any nonzero element $x$ of $\mathbb{K}$ can be written as

$$
x=\pi^{o r d(x)} \sum_{i=0}^{\infty} x_{i} \pi^{i}
$$

where $x_{i} \in \mathfrak{S}$ and $x_{0} \neq 0$. This series converges in the norm $|\cdot|_{\mathbb{K}}$.

We extend the norm $|\cdot|_{\mathbb{K}}$ to $\mathbb{K}^{n}$ by taking

$$
\|\left. x\right|_{\mathbb{K}}:=\max _{1 \leq i \leq n}\left|x_{i}\right|_{\mathbb{K}}
$$

for $x=\left(x_{1}, \ldots, x_{n}\right) \in \mathbb{K}^{n}$.We define $\operatorname{ord}(x)=\min _{1 \leq i \leq n}\left\{\operatorname{ord}\left(x_{i}\right)\right\}$, then $\|x\|_{\mathbb{K}}=q^{-\operatorname{ord}(x)}$. The metric space $\left(\mathbb{K}^{n},\|\cdot\|_{\mathbb{K}}\right)$ is a complete ultrametric space.

As we mentioned before, any finite extension $\mathbb{K}$ of $\mathbb{Q}_{p}$ is a non-Archimedean local field. Then

$$
p R_{\mathbb{K}}=\pi^{m} R_{\mathbb{K}}, \quad m \in \mathbb{N} .
$$


If $m=1$ we say that $\mathbb{K}$ is a unramified extension of $\mathbb{Q}_{p}$. In other case, we say that $\mathbb{K}$ is a ramified extension. It is well known that for every positive integer $e$ there exists a unique unramified extension $\mathbb{K}_{e}$ of $\mathbb{Q}_{p}$ of degree $e$, which means that $\mathbb{K}_{e}$ is a $\mathbb{Q}_{p}$-vector space of dimension $e$. From now on, $\pi$ denotes a local uniformizing parameter of $\mathbb{K}_{e}$, thus $p R_{\mathbb{K}_{e}}=\pi R_{\mathbb{K}_{e}}, R_{\mathbb{K}_{e}} / P_{\mathbb{K}_{e}} \cong \mathbb{F}_{p^{e}}$ and $|\pi|_{\mathbb{K}_{e}}=p^{-e}$. For an in-depth exposition of non-Archimedean local fields, the reader may consult [62,65], see also [3,61].

\subsection{Open String Amplitudes over Non-Archimedean Local Fields}

The open string amplitudes can be defined over any local field. In this section, we consider Koba-Nielsen string amplitudes on $\mathbb{K}_{e}$, the unique unramified extension of $\mathbb{Q}_{p}$ of degree $e$ for all $e \in \mathbb{N} \backslash\{0\}$. We recall that if $\mathbb{K}_{e}$ is the unramified extension of degree $e$ of $\mathbb{Q}_{p}$, then $p R_{\mathbb{K}_{e}}=\pi R_{\mathbb{K}_{e}}, R_{\mathbb{K}_{e}} / P_{\mathbb{K}_{e}} \cong \mathbb{F}_{p^{e}}$ and $|\pi|_{\mathbb{K}_{e}}=p^{-e}$. Thus, $\pi$ in $\mathbb{K}_{e}$ plays the role of $p$ in $\mathbb{Q}_{p}$.

The Koba-Nielsen amplitudes on $\mathbb{K}_{e}$ are defined as

$$
\boldsymbol{A}_{\mathbb{K}_{e}}^{(N)}(\boldsymbol{k})=\int_{\mathbb{K}_{e}^{N-3}} \prod_{i=2}^{N-2}\left|x_{i}\right|_{\mathbb{K}_{e}}^{\boldsymbol{k}_{1} k_{i}}\left|1-x_{i}\right|_{\mathbb{K}_{e}}^{\boldsymbol{k}_{N-1} \boldsymbol{k}_{i}} \prod_{2 \leq i<j \leq N-2}\left|x_{i}-x_{j}\right|_{\mathbb{K}_{e}}^{\boldsymbol{k}_{i} \boldsymbol{k}_{j}} \prod_{i=2}^{N-2} d x_{i},
$$

where $\prod_{i=2}^{N-2} d x_{i}$ is the Haar measure of $\left(\mathbb{K}_{e}^{N-3},+\right)$ normalized so that the measure of $R_{\mathbb{K}_{e}}^{N-3}$ is 1 .

The procedure used to regularize the $p$-adic amplitudes extends to amplitudes of the form (51). In this case, the open string $N$-point zeta function is defined as

$$
\mathbf{Z}_{\mathbb{K}_{e}}^{(N)}(s):=\int_{\mathbb{K}_{e}^{N-3}} F\left(s, x ; N, \mathbb{K}_{e}\right) \prod_{i=2}^{N-2} d x_{i}
$$

where

$$
F\left(s, x ; N, \mathbb{K}_{e}\right)=\prod_{i=2}^{N-2}\left|x_{i}\right|_{\mathbb{K}_{e}}^{s_{1 i}}\left|1-x_{i}\right|_{\mathbb{K}_{e}}^{s_{(N-1) i}} \prod_{2 \leq i<j \leq N-2}\left|x_{i}-x_{j}\right|_{\mathbb{R}_{e}}^{s_{i j}} .
$$

where $\underline{s}=\left(s_{i j}\right) \in \mathbb{C}^{D}$, with $D=\frac{(N-3)(N-4)}{2}+2(N-3)$. Let $T=\{2,3, \ldots, N-2\}$, then

$$
Z_{\mathbb{K}_{e}}^{(N)}(s)=\sum_{I \subseteq T} p^{e M(\underline{s})} Z_{\mathbb{K}_{e}}^{(N)}(s ; I, 0) Z_{\mathbb{K}_{e}}^{(N)}(s ; T \backslash I, 1),
$$

where

$$
\begin{gathered}
M(s):=|T \backslash I|+\sum_{i \in T \backslash I}\left(s_{1 i}+s_{(N-1) i}\right)+\sum_{\substack{2 \leq i<j \leq N-2 \\
i \in T \backslash I, j \in T}} s_{i j}+\sum_{\substack{2 \leq i<j \leq N-2 \\
i \in I, j \in T \backslash I}} s_{i j} \\
\mathbf{Z}_{\mathbb{K}_{e}}^{(N)}(s ; I, 0)=\int_{R_{\mathbb{K}_{e}}^{I I}} \prod_{i \in I}\left|x_{i}\right|_{\mathbb{K}_{e}}^{s_{1 i}}\left|1-x_{i}\right|_{\mathbb{K}_{e}}^{s_{(N-1) i}} \prod_{\substack{2 \leq i<j \leq N-2 \\
i, j \in I}}\left|x_{i}-x_{j}\right|_{\mathbb{K}_{e}}^{s_{i j}} \prod_{i \in I} d x_{i},
\end{gathered}
$$

and

$$
Z_{\mathbb{K}_{e}}^{(N)}(s ; T \backslash I, 1,)=\int_{\substack{|T \backslash \backslash| \\ R_{\mathbb{K}_{e}}}} F_{1}\left(s, x ; N, \mathbb{K}_{e}\right) \prod_{i \in T \backslash I} d x_{i},
$$

where 


$$
F_{1}\left(s, x ; N, \mathbb{K}_{e}\right):=\frac{\prod_{\substack{2 \leq i<j \leq N-2 \\ i, j \in T \backslash I}}\left|x_{i}-x_{j}\right|_{\mathbb{K}_{e}}^{s_{i j}}}{\prod_{i \in T \backslash I}\left|x_{i}\right|_{\mathbb{K}_{e}}^{2+s_{1 i}+s_{(N-1) i}+\sum_{2 \leq j \leq N-2, j \neq i} s_{i j}}} .
$$

By convention $\boldsymbol{Z}_{\mathbb{K}_{e}}^{(N)}(\boldsymbol{s} ; \varnothing, 0)=1, \boldsymbol{Z}_{\mathbb{K}_{e}}^{(N)}(s ; \varnothing, 1)=1$.

All the zeta functions appearing in the right-hand side of Formula (53) admit analytic continuations to the whole $\mathbb{C}^{D}$ as rational functions in the variables $p^{-e s_{i j}}$ and they are holomorphic on a common domain in $\mathbb{C}^{D}$. Therefore $\boldsymbol{Z}_{\mathbb{K}_{e}}^{(N)}(s)$ is a holomorphic function in a certain domain of $\mathbb{C}^{D}$ admitting a meromorphic continuation to the whole $\mathbb{C}^{D}$ as a rational function in the variables $p^{-e s_{i j}}$, see Theorem 1 of [8].

We use $\boldsymbol{Z}_{\mathbb{K}_{e}}^{(N)}(\boldsymbol{s})$ as regularizations of Koba-Nielsen amplitudes $A^{(N)}\left(\boldsymbol{k}, \mathbb{K}_{e}\right)$, more precisely, we define

$$
\boldsymbol{A}_{\mathbb{K}_{e}}^{(N)}(\boldsymbol{k})=\left.\boldsymbol{Z}_{\mathbb{K}_{e}}^{(N)}(s)\right|_{s_{i j}=\boldsymbol{k}_{i} \cdot \boldsymbol{k}_{j}} .
$$

Then $\boldsymbol{A}_{\mathbb{K}_{e}}^{(N)}(\boldsymbol{k})$ is a well-defined rational function in the variables $p^{-e \boldsymbol{k}_{i} \cdot \boldsymbol{k}_{j}}$, which agree with the integral (51) when it converges.

\subsection{The Limit $p$ Tends to One}

The functions

$$
\boldsymbol{Z}_{\mathbb{K}_{e}}^{(N)}(\boldsymbol{s} ; I, 0), \boldsymbol{Z}_{\mathbb{K}_{e}}^{(N)}(s ; T \backslash I, 1)
$$

are multivariate local zeta functions. Thus, to make mathematical sense of the limit of $Z_{\mathbb{Q}_{p}}^{(N)}(s)$ as $p \rightarrow 1$ we use the work of Denef and Loeser, see [35,66], and compute the limit of $\boldsymbol{Z}_{\mathbb{K}_{e}}^{(N)}(s)$ as $e \rightarrow 0$ instead of the limit of $\boldsymbol{Z}_{\mathbb{Q}_{p}}^{(N)}(s)$ as $p \rightarrow 1$. In order to compute the limit $e \rightarrow 0$ is necessary to have an explicit formula for $Z_{\mathbb{K}_{e}}^{(N)}(s)$, so in [9] we determined the explicit formula by finding explicit formulae for integrals $\boldsymbol{Z}_{\mathbb{K}_{e}}^{(N)}(s ; I, 0)$ and $\boldsymbol{Z}_{\mathbb{K}_{e}}^{(N)}(s ; T \backslash I, 1)$, see Theorem B in [9]. After that, we define

$$
Z_{\text {top }}^{(N)}(s ; I, 0)=\lim _{e \rightarrow 0} Z_{\mathbb{K}_{e}}^{(N)}(s ; I, 0)
$$

and

$$
Z_{\text {top }}^{(N)}(s ; T \backslash I, 1)=\lim _{e \rightarrow 0} Z_{\mathbb{K}_{e}}^{(N)}(s ; T \backslash I, 1),
$$

which are elements of $\mathbb{Q}\left(s_{i j}, i, j \in\{1, \ldots, N-1\}\right)$, the field of rational functions in the variables $s_{i j}, i, j \in\{1, \ldots, N-1\}$ with coefficients in $\mathbb{Q}$. Then, by using (53), we defined the open string $N$-point topological zeta function as

$$
\boldsymbol{Z}_{\text {top }}^{(N)}(\boldsymbol{s})=\sum_{I \subseteq T} \boldsymbol{Z}_{\text {top }}^{(N)}(\boldsymbol{s} ; I, 0) \mathbf{Z}_{\text {top }}^{(N)}(s ; T \backslash I, 1) .
$$

The open string $N$-point topological zeta function $Z_{\text {top }}^{(N)}(\boldsymbol{s})$ is a rational function of $\mathbb{Q}\left(s_{i j}, i, j \in\{1, \ldots, N-1\}\right)$. We now define the Denef-Loeser open string $N$-point amplitudes at the tree level as

$$
\boldsymbol{A}_{\text {top }}^{(N)}(\boldsymbol{k})=\left.\mathbf{Z}_{\text {top }}^{(N)}(\boldsymbol{s})\right|_{s_{i j}=\boldsymbol{k}_{i} \cdot \boldsymbol{k}_{j}},
$$

with $i \in\{1, \ldots, N-1\}, j \in T$ or $i, j \in T$, where $T=\{2, \ldots, N-2\}$. Thus, the Denef-Loeser amplitudes are rational functions of the variables $\boldsymbol{k}_{i} \cdot \boldsymbol{k}_{j}, i, j \in\{1, \ldots, N\}$. 


\subsection{Feynman Rules, Explicit Formulae and Denef-Loeser Amplitudes}

Using the Feynman rules of the effective Lagrangian, an 'explicit formula' of the type

$$
\boldsymbol{A}^{(N)}(\boldsymbol{k})=K_{N}+\sum_{l=2}^{N-3} K_{i_{1} \ldots i_{l}} \sum_{1 \leq a<b \leq l}\left(\frac{p-1}{p} \frac{p^{-k_{i_{a}} k_{i_{b}}-1}}{1-p^{-k_{i_{a}} k_{i_{b}}-1}}\right)
$$

was given in ([14]), where the constants $K_{N}, K_{i_{1} \ldots i_{l}} \in \mathbb{Q}(p)$. Of course, a rigorous demonstration of a such formula is an open problem. Taking formally the limit $p$ tends to one, we get

$$
\boldsymbol{A}_{\text {top }}^{(N)}(\boldsymbol{k})=K_{N}^{\text {top }}+\sum_{l=2}^{N-3} K_{i_{1} \ldots i_{l}}^{\text {top }} \sum_{1 \leq a<b \leq l} \frac{1}{k_{i_{a}} k_{i_{b}}+1} .
$$

\subsection{Denef-Loeser Open String 4-Point Amplitudes}

The open string 4-point zeta function on $\mathbb{K}_{e}$ is defined as

$$
\mathbf{Z}_{\mathbb{K}_{e}}^{(4)}(s)=\int_{\mathbb{K}_{e}}\left|x_{2}\right|_{\mathbb{K}_{e}}^{s_{12}}\left|1-x_{2}\right|_{\mathbb{K}_{e}}^{s_{32}} d x_{2}
$$

We divide the integration domain in sectors

$$
\operatorname{Sect}(I)=\left\{x_{i} \in \mathbb{Q}:\left|x_{i}\right|_{\mathbb{K}_{e}} \leq 1 \Longleftrightarrow i \in I\right\}, I \subseteq T=\{2,3\},
$$

\begin{tabular}{ccc}
\hline $\mathbf{I}$ & $\mathbf{T} \backslash \mathbf{I}$ & $\operatorname{Sect}(\mathbf{I})$ \\
\hline$\{2\}$ & $\varnothing$ & $R_{\mathbb{K}_{e}}$ \\
\hline$\varnothing$ & $\{2\}$ & $K_{e} \backslash R_{\mathbb{K}_{e}}$. \\
\hline
\end{tabular}

In this way, we obtain that

$$
\begin{aligned}
\mathbf{Z}_{\mathbb{K}_{e}}^{(4)}(s) & =\boldsymbol{Z}_{\mathbb{K}_{e}}^{(4)}(s ;\{2\}, 0)+p^{e\left(1+s_{12}+s_{32}\right)} \mathbf{Z}_{\mathbb{K}_{e}}^{(4)}(\underline{s}\{2\}, 1) \\
& =\int_{R_{\mathbb{K}_{e}}}\left|x_{2}\right|_{\mathbb{K}_{e}}^{s_{12}}\left|1-x_{2}\right|_{\mathbb{K}_{e}}^{s_{32}} d x_{2}+p^{e\left(1+s_{12}+s_{32}\right)} \int_{R_{\mathbb{K}_{e}}}\left|x_{2}\right|_{\mathbb{K}_{e}}^{-2-s_{12}-s_{32}} d x_{2},
\end{aligned}
$$

with

$$
\boldsymbol{Z}_{\mathbb{K}_{e}}^{(4)}(s ;\{2\}, 0)=1-2 p^{-e}+\frac{\left(1-p^{-e}\right) p^{e\left(-1-s_{12}\right)}}{1-p^{e\left(-1-s_{12}\right)}}+\frac{\left(1-p^{-e}\right) p^{e\left(-1-s_{32}\right)}}{1-p^{e\left(-1-s_{32}\right)}}
$$

and

$$
Z_{\mathbb{K}_{e}}^{(4)}(s ;\{2\}, 1)=\frac{\left(1-p^{-e}\right) p^{e\left(1+s_{12}+s_{32}\right)}}{1-p^{e\left(1+s_{12}+s_{32}\right)}} .
$$

Taking the limit $e$ approaches to zero,

$$
Z_{\text {top }}^{(4)}(s ;\{2\}, 0)=-1+\frac{1}{s_{12}+1}+\frac{1}{s_{32}+1}
$$

and

$$
\mathbf{Z}_{\text {top }}^{(4)}(s ;\{2\}, 1)=-\frac{1}{s_{12}+s_{32}+1} .
$$

Consequently

$$
Z_{\text {top }}^{(4)}(s)=-1+\frac{1}{s_{12}+1}+\frac{1}{s_{32}+1}-\frac{1}{s_{12}+s_{32}+1} .
$$


Using the kinematic relations $\boldsymbol{k}_{1}+\boldsymbol{k}_{2}+\boldsymbol{k}_{3}+\boldsymbol{k}_{4}=0$ and $\boldsymbol{k}_{i}^{2}=2$ we obtain $\boldsymbol{k}_{1} \boldsymbol{k}_{2}+$ $k_{3} k_{2}+1=-1-k_{2} k_{4}$, then the Denef-Loeser string 4-point amplitude is given by

$$
A_{\text {top }}^{(4)}(k)=-1+\frac{1}{k_{1} k_{2}+1}+\frac{1}{k_{3} k_{2}+1}+\frac{1}{k_{2} k_{4}+1} .
$$

\subsection{Denef-Loeser Open String 5-Point Amplitudes}

The open string 5-point zeta function on $\mathbb{K}_{e}$ is given by

$$
Z_{\mathbb{K}_{e}}^{(5)}(s)=\int_{\mathbb{K}_{e}^{2}}\left|x_{2}\right|_{\mathbb{K}_{e}}^{s_{12}}\left|x_{3}\right|_{\mathbb{K}_{e}}^{s_{13}}\left|1-x_{2}\right|_{\mathbb{K}_{e}}^{s_{42}}\left|1-x_{3}\right|_{\mathbb{K}_{e}}^{s_{4}}\left|x_{2}-x_{3}\right|_{\mathbb{K}_{e}}^{s_{23}} d x_{2} d x_{3} .
$$

By dividing the integration domain in sectors as in Section 4.2, we obtain the results presented in Table 2.

Table 2. Sectors of the 5-point Denef-Loeser amplitude.

\begin{tabular}{ccc}
\hline $\mathbf{I}$ & $\mathbf{I}^{\mathbf{c}}$ & Sect $(\mathbf{I})$ \\
\hline$\{2\}$ & $\{3\}$ & $R_{\mathbb{K}_{e}} \times \mathbb{K}_{e} \backslash R_{\mathbb{K}_{e}}$ \\
\hline$\{3\}$ & $\{2\}$ & $\mathbb{K}_{e} \backslash R_{\mathbb{K}_{e}} \times R_{\mathbb{K}_{e}}$ \\
\hline$\{2,3\}$ & $\varnothing$ & $R_{\mathbb{K}_{e}} \times R_{\mathbb{K}_{e}}$ \\
\hline$\varnothing$ & $\{2,3\}$ & $\mathbb{K}_{e} \backslash R_{\mathbb{K}_{e}} \times \mathbb{K}_{e} \backslash R_{\mathbb{K}_{e}}$. \\
\hline
\end{tabular}

The open string 5-point topological zeta function is defined as

$$
\mathbf{Z}_{\text {top }}^{(5)}(s)=\sum_{I \subseteq T} \boldsymbol{Z}_{\text {top }}^{(5)}(s ; I, 0) Z_{\text {top }}^{(5)}(s ; T \backslash I, 1) .
$$

\begin{tabular}{|c|c|c|}
\hline I & $Z_{t o p}^{(5)}(s ; I, 0)$ & $Z_{t o p}^{(5)}(\underline{s} ; T \backslash I, 1)$ \\
\hline$\{2\}$ & $-1+\frac{1}{1+s_{12}}+\frac{1}{1+s_{42}}$ & $-\frac{1}{1+s_{13}+s_{43}+s_{23}}$ \\
\hline$\{3\}$ & $-1+\frac{1}{1+s_{13}}+\frac{1}{1+s_{43}}$ & $-\frac{1}{1+s_{12}+s_{42}+s_{23}}$ \\
\hline$\{2,3\}$ & $\begin{array}{l}{\left[\frac{1}{1+s_{12}}+\frac{1}{1+s_{13}}+\frac{1}{1+s_{23}}-1\right] \frac{1}{2+s_{12}+s_{13}+s_{23}}} \\
+\frac{1}{1+s_{12}}\left[\frac{1}{1+s_{43}}-1\right]+\frac{1}{1+s_{13}}\left[\frac{1}{1+s_{42}}-1\right] \\
\quad+2-\frac{1}{1+s_{23}}-\frac{1}{1+s_{42}}-\frac{1}{1+s_{43}}+ \\
\quad \frac{1}{1+s_{42}}+\frac{1}{1+s_{43}+s_{23}}\left[\frac{1}{1+s_{42}}+\frac{1}{1+s_{43}}+\frac{1}{1+s_{23}}-1\right]\end{array}$ & 1 \\
\hline$\{\varnothing\}$ & $x$ & $\begin{array}{c}-\frac{1}{2+s_{52}+s_{53}+s_{23}} \\
\frac{1}{1+s_{12}+s_{42}+s_{23}}+\frac{1}{1+s_{13}+s_{43}+s_{23}} \\
+\frac{1+s_{23}}{1+1}\end{array}$ \\
\hline
\end{tabular}

Table 3 contains explicit formulae for all the integrals $Z_{\text {top }}^{(5)}(s ; I, 0)$ and $Z_{\text {top }}^{(5)}(s ; T \backslash I, 1)$.

Table 3. Explicit calculation of $\boldsymbol{Z}_{\text {top }}^{(5)}(s ; I, 0), Z_{t o p}^{(5)}(s ; T \backslash I, 1)$.

Therefore, the Denef-Loeser open string 5-point amplitude is given by

$$
\boldsymbol{A}_{\text {top }}^{(5)}(\boldsymbol{k})=2-\sum_{i<j} \frac{1}{1+\boldsymbol{k}_{i} \boldsymbol{k}_{j}}+\sum_{\substack{\{i, j, k, l\} \subset\{1,2,3,4,5\} \\ i<j, k<l}} \frac{1}{1+\boldsymbol{k}_{i} \boldsymbol{k}_{j}} \frac{1}{1+\boldsymbol{k}_{k} \boldsymbol{k}_{l}},
$$


where

$$
\begin{gathered}
\sum_{\substack{\{i, j, k, l\} \subset\{1,2,3,4,5\} \\
i<j, k<l}} \frac{1}{1+k_{i} k_{j}} \frac{1}{1+k_{k} k_{l}} \\
=\frac{1}{1+k_{1} k_{2}} \frac{1}{1+k_{3} k_{4}}+\frac{1}{1+k_{1} k_{2}} \frac{1}{1+k_{3} k_{5}}+\frac{1}{1+k_{1} k_{2}} \frac{1}{1+k_{4} k_{5}} \\
+\frac{1}{1+k_{1} k_{3}} \frac{1}{1+k_{2} k_{4}}+\frac{1}{1+k_{1} k_{3}} \frac{1}{1+k_{2} k_{5}}+\frac{1}{1+k_{1} k_{3}} \frac{1}{1+k_{4} k_{5}} \\
+\frac{1}{1+k_{1} k_{4}} \frac{1}{1+k_{2} k_{3}}+\frac{1}{1+k_{1} k_{4}} \frac{1}{1+k_{2} k_{5}}+\frac{1}{1+k_{1} k_{4}} \frac{1}{1+k_{3} k_{5}} \\
+\frac{1}{1+k_{1} k_{5}} \frac{1}{1+k_{2} k_{3}}+\frac{1}{1+k_{1} k_{5}} \frac{1}{1+k_{2} k_{4}}+\frac{1}{1+k_{1} k_{5}} \frac{1}{1+k_{3} k_{4}} \\
+\frac{1}{1+k_{2} k_{3}} \frac{1}{1+k_{4} k_{5}}+\frac{1}{1+k_{3} k_{5}} \frac{1}{1+k_{2} k_{4}}+\frac{1}{1+k_{2} k_{5}} \frac{1}{1+k_{3} k_{4}}
\end{gathered}
$$

This amplitude agrees with the calculation

$$
\begin{aligned}
A_{\text {top }}^{(5)}(\boldsymbol{k}) & =\lim _{p \rightarrow 1} \boldsymbol{A}^{(5)}(\boldsymbol{k}) \\
= & K_{5}^{\text {top }}+K_{4}^{\text {top }} \sum_{i<j} \frac{1}{\boldsymbol{k}_{i} \boldsymbol{k}_{j}+1}+\sum_{\{i, j, k, l\} \subset\{1,2,3,4,5\}} \frac{1}{\boldsymbol{k}_{i} \boldsymbol{k}_{j}+1} \frac{1}{\boldsymbol{k}_{k} \boldsymbol{k}_{l}+1}
\end{aligned}
$$

done using the explicit formula for given by the Feynman rules given in [14]. Here $K_{5}^{\text {top }}=-2, K_{4}^{\text {top }}=-1$.

\subsection{Non-Archimedean Closed Strings}

In Archimedean string theory, it is known that closed strings can be produced from the scattering of open strings [55]. Therefore, for $p$-adic string theory, it is desirable to construct a $p$-adic closed string theory. This was first studied in [20], see also [4,67].

The usual Archimedean closed string is described by two coordinates $(\tau, \sigma)$ with the periodical condition $\sigma^{\prime}=\sigma+2 \pi$. That is, the string worldsheet is a cylinder, which can be conformally mapped to the whole complex plane $\mathbb{C}$. In order to obtain tree-level scattering amplitudes we need to insert vertex operators on $\mathbb{C}$. Using the $\operatorname{SL}(2, \mathbb{C})$ symmetry, we can fix three insertions points. As with the open string, it is conventional to fix three points to 0,1 and $\infty$. The simplest non-trivial example is the 4-point tachyon scattering amplitude for closed strings, also known as the Virasoro-Shapiro amplitude, is

$$
A_{\mathbb{C}}^{(4)}(\boldsymbol{k})=\int_{\mathbb{C}}|z|_{\mathbb{C}}^{k_{1} k_{2} / 4}|1-z|_{\mathbb{C}}^{k_{1} k_{3} / 4} d z=\frac{\Gamma_{\mathbb{C}}(-\alpha(s) / 2) \Gamma_{\mathbb{C}}(-\alpha(t) / 2)}{\Gamma_{\mathbb{C}}(-\alpha(s) / 2-\alpha(t) / 2)},
$$

where $\boldsymbol{k}_{i} \in \mathbb{C}^{D}$ and $\boldsymbol{k}_{i} \boldsymbol{k}_{j}=-k_{0, i} k_{0, j}+\cdots k_{l, i} k_{l, j}$ is the Minkowski product; $|z|_{\mathbb{C}}:=z \bar{z}$ is the square complex norm, $\alpha(x)=x / 4+2$, and

$$
\Gamma_{\mathbb{C}}(s):=\int_{\mathbb{C}} \exp [2 \pi i(z+\bar{z})]|z|_{\mathbb{C}}^{s-1} d z
$$

is the Gelfand-Graev gamma function over $\mathbb{C}$ [4]. For the closed strings case, the momenta vectors satisfy

$$
\boldsymbol{k}_{i}^{2}=8, \quad \sum_{i=1}^{N} \boldsymbol{k}_{i}=0
$$


This explains the factor of $1 / 4$ in the exponents relative to the open strings case. The generalization of these amplitudes to $N$-points is given by

$$
A_{\mathbb{C}}^{(N)}(\boldsymbol{k})=\int_{\mathbb{C}^{N-3}} \prod_{i=2}^{N-2}\left|z_{i}\right|_{\mathbb{C}}^{k_{1} k_{i} / 4}\left|1-z_{i}\right|_{\mathbb{C}}^{k_{N-1} k_{i} / 4} \prod_{2 \leq i<j \leq N-2}\left|z_{i}-z_{j}\right|_{\mathbb{C}}^{k_{i} k_{j} / 4} \prod_{i=2}^{N-2} d z_{i},
$$

where $\boldsymbol{k}=\left(\boldsymbol{k}_{1}, \ldots, \boldsymbol{k}_{N}\right)$. As we can see, these amplitudes are very similar to the KobaNielsen amplitudes, except for the fact that they are being integrated over complex variables and the replacement $k_{i} \rightarrow \frac{1}{2} k_{i}$.

To construct $p$-adic versions of Virasoro-Shapiro amplitudes, one can consider $\mathbb{Q}_{p}$ as the analog of $\mathbb{R}$, and a quadratic extension of $\mathbb{Q}_{p}$ as the analog of $\mathbb{C}$. However, this quadratic extension is not unique, and is not an algebraically closed field. This naive approach is followed in $[4,20]$. This approach is not very useful here, because we work with open string amplitudes over any finite extension of $\mathbb{Q}_{p}$.

Definition 2. Given an open string N-point amplitude $A_{\mathbb{K}}^{(N)}(\boldsymbol{k})$ defined over a non-Archimedean local field $\mathbb{K}$, we attach to it a Virasoro-Shapiro amplitude defined as $\mathcal{A}_{\mathbb{K}_{2}}^{(N)}(\boldsymbol{k})=A_{\mathbb{K}_{2}}^{(N)}\left(\frac{1}{2} \boldsymbol{k}\right)$, where $\mathbb{K}_{2}$ is the unique unramified extension of $\mathbb{K}$ of degree 2.

Then, by the results of [6], $\mathcal{A}_{\mathbb{K}_{2}}^{(N)}(\boldsymbol{k})$ admits a meromorphic continuation as a rational function in the variables $q^{-2 s_{i j}}$.

\section{A Physical View of the Limit $p \rightarrow 1$ on $p$-Adic String Amplitudes}

The problem of finding a relation between the physical string amplitudes and the $p$-adic one has been present since the initial proposal. One of the first attempts is that of the Adelic amplitudes which can be written as an infinite product of physical non-Archimedean amplitudes [14,21].

There are other possibilities to look for a link between Archimedean and Non-Archimedean amplitudes. One of them is to consider the limit $p \rightarrow 1$. This limit is very intriguing and there are different interpretations of it [23,29,34]. For instance, in [23] it was argued that the analytic continuation of $p$ to the complex numbers can be reflected in the Lagrangian and equations of motion by turning the non-local equations of motion into a local and linear dynamical description with a logarithmic potential. This is an ordinary theory with real physical amplitudes. In [29] it was argued that as similarly found in [23], the expansion of the effective action around $p \rightarrow 1$ leads to a linear theory with logarithmic potential. Besides that, this theory is deeply related to the boundary string field theory proposed by Witten [30] in the context of the developing of a background independence string theory. The limit $p \rightarrow 1$ also can be interpreted in terms of some scaling transformations of the renormalization group for the Bruhat-Tits tree [34].

As we have seen in this survey, the non-Archimedean nature is encoded in the worldsheet theory. In this context we must recall that $p$ as a prime number, thus the analysis necessarily needs to be carry out in a rigorous way. The right way of taking the limit $p \rightarrow 1$ involves the introduction of unramified finite extensions of the $p$-adic field $\mathbb{Q}_{p}$. In [9] the limit $p \rightarrow 1$ was discussed for tree-level string amplitudes, by using the topological zeta functions introduced by Denef and Loeser $[35,66]$. We found that the limit $p \rightarrow 1$ of $p$-adic string amplitudes leads to certain string amplitudes (which are rational functions) that we termed the Denef-Loeser open string amplitudes. These Denef-Loeser amplitudes for $N=4,5$ points were computed in Sections 5.5 and 5.6, for more details see [9]. On the other hand for $N=4,5$, in [9], we computed the limit $p \rightarrow 1$ of the effective field theory of $p$-adic amplitudes, i.e., the Gerasimov and Shatashvili Lagrangian (61) involving a logarithmic potential. By computing the interacting generating functional at the tree level, we verified that the corresponding amplitudes coincide exactly with the Denef-Loeser 
amplitudes. Based on this fact we have formulated a conjecture, which was already stated in the introduction of this survey.

In what follows we review the evidence of the mentioned conjecture in the cases $N=4,5$ following [9]. First, we review some basic results from [29] starting with the effective action which is a field theory whose perturbative analysis leads to the $p$-adic scattering amplitudes [14], it can be written as

$$
S(\phi)=\frac{1}{g^{2}} \frac{p^{2}}{p-1} \int d^{D} x\left(-\frac{1}{2} \phi p^{-\frac{1}{2} \Delta} \phi+\frac{1}{p+1} \phi^{p+1}\right),
$$

where $\Delta$ is the Laplacian on $M$ and $g$ is the coupling constant. The corresponding EulerLagrange equation is

$$
p^{-\frac{1}{2} \Delta} \phi=\phi^{p}
$$

In the source space (and consequently in the amplitudes) $p$ is a prime number; however, in the target space (and consequently in the Lagrangian and in the equation of motion) $p$ is a real parameter. Thus, one can formally proceed to approach $p$ to one and perform a Taylor expansion at $(p-1)$ of the expression $\exp \left(-\frac{1}{2} \Delta \log p\right)$ and $\exp (p \log \phi)$. Then the resulting equation of motion is given by

$$
\Delta \phi=-2 \phi \log \phi .
$$

This is the motion equation of the Gerasimov-Shatashvili action

$$
S(\phi)=\int d^{D} x\left((\partial \phi)^{2}-V(\phi)\right)
$$

where $(\partial \phi)^{2}=\eta^{\mu v} \partial_{\mu} \phi \cdot \partial_{\nu} \phi$ and $V(\phi)$ is the potential

$$
V(\phi)=\phi^{2} \log \frac{\phi^{2}}{e} .
$$

We consider that action (61) is the limit $p$ tends to one of the effective action (58).

The action corresponding to the free theory with a source is written as

$$
S_{0}(\phi)=\int d^{D} x\left[(\partial \phi)^{2}+\phi^{2}(x)+J(x) \phi(x)\right] .
$$

Now, the action (61) can be conveniently rewritten as

$$
S(\phi)=\int d^{D} x\left[(\partial \phi)^{2}+m^{2}-U(\phi)\right],
$$

where $U(\phi)=2 \phi^{2} \log \phi$. Then the potential $U(\phi)$ can be expanded in Taylor series around the origin in the form

$$
U(\phi)=A \phi^{2}+B \phi^{3}+C \phi^{4}+D \phi^{5}+\cdots,
$$

where $A, B, C$ and $D$ are constants and they are real numbers.

Now, we briefly review the form of obtaining the four-point and five-point amplitudes from the Lagrangian (61) [29]. In quantum field theory $N$-point correlation functions of $N$ local operators $\widehat{\phi}$ in $N$ different points $x_{1}, x_{2}, \ldots, x_{N}$ of $M$, can be written as

$$
\left\langle T\left(\widehat{\phi}\left(x_{1}\right) \widehat{\phi}\left(x_{2}\right) \cdots \widehat{\phi}\left(x_{N}\right)\right)\right\rangle=\left.\frac{(-i \hbar)^{N}}{\mathcal{Z}[J]} \frac{\delta^{n} \mathcal{Z}[J]}{\delta J\left(x_{1}\right) \delta J\left(x_{2}\right) \cdots \delta J\left(x_{N}\right)}\right|_{J=0},
$$


where $\mathcal{Z}[J]$ is the generating functional constructed using interacting Lagrangian (62). The functional can be computed as

$$
\begin{aligned}
\mathcal{Z}[J]=\exp \{- & \frac{i B}{\hbar} \int d^{D} x\left(-i \hbar \frac{\delta}{\delta J(x)}\right)^{3} \\
& \left.-\frac{i C}{\hbar} \int d^{D} x\left(-i \hbar \frac{\delta}{\delta J(x)}\right)^{4}-\frac{i D}{\hbar} \int d^{D} x\left(-i \hbar \frac{\delta}{\delta J(x)}\right)^{5}+\cdots\right\} \mathcal{Z}_{0}[J],
\end{aligned}
$$

where the generating functional of the correlation function for the free theory with sources is given by

$$
\mathcal{Z}_{0}[J]=\mathcal{N}[\operatorname{det}(\Delta-1)]^{-1 / 2} \exp \left\{-\frac{i}{4 \hbar} \int d^{D} x \int d^{D} x^{\prime} J(x) G_{F}\left(x-x^{\prime}\right) J\left(x^{\prime}\right)\right\} .
$$

Moreover, $G_{F}\left(x-x^{\prime}\right)$ is the Green-Feynman function of the differential operator $(\Delta-1)$.

For the computation of the interacting 4-point amplitudes can be obtained through the generating functional

$$
\mathcal{Z}[J]=\cdots-i C \hbar^{3} \int d^{D} x\left(\frac{\delta}{\delta J(x)}\right)^{4} \mathcal{Z}_{0}[J]+\cdots .
$$

In terms of $\mathcal{Z}[J], 4$-point amplitudes implies the computation of

$$
\begin{gathered}
\left.\frac{\delta^{4} \mathcal{Z}[J]}{\delta J\left(x_{1}\right) \delta J\left(x_{2}\right) \delta J\left(x_{3}\right) \delta J\left(x_{4}\right)}\right|_{J=0} \\
=-4 ! i C \hbar^{3} \int d^{D} x\left[-\frac{i}{2 \hbar} G_{F}\left(x-x_{1}\right)\right]\left[-\frac{i}{2 \hbar} G_{F}\left(x-x_{2}\right)\right] \\
\times\left[-\frac{i}{2 \hbar} G_{F}\left(x-x_{3}\right)\right]\left[-\frac{i}{2 \hbar} G_{F}\left(x-x_{4}\right)\right] \\
=-\frac{3 i C}{2 \hbar} \int d^{D} x G_{F}\left(x-x_{1}\right) G_{F}\left(x-x_{2}\right) G_{F}\left(x-x_{3}\right) G_{F}\left(x-x_{4}\right) .
\end{gathered}
$$

Here $G_{F}(x-y)$ is the Green-Feynman propagator. This amplitude corresponds to the Feynman diagram with only one vertex and four external legs. following the notation of [14], we write for it the symbol $\bar{K}_{4}$.

The contribution to the 4-point amplitudes of $B \phi^{3}$ in (63) has a contribution at the second order of the expansion. The corresponding Feynman diagrams have two vertices at points $x$ and $y$ with two external legs, which are connected by a propagator $G_{F}(x-y)$. Thus, we have

$$
\mathcal{Z}[J]=\cdots+\frac{B^{2} \hbar^{4}}{2} \int d^{D} x \int d^{D} y\left(\frac{\delta}{\delta J(x)}\right)^{3}\left(\frac{\delta}{\delta J(y)}\right)^{3} \mathcal{Z}_{0}[J]+\cdots .
$$

Moreover, the contribution to the 4-point amplitudes from the term $C \phi^{3}$ arises to second order. Thus, it yields

$$
\begin{gathered}
\left.\frac{\delta^{4} \mathcal{Z}[J]}{\delta J\left(x_{1}\right) \delta J\left(x_{2}\right) \delta J\left(x_{3}\right) \delta J\left(x_{4}\right)}\right|_{J=0}=18 B^{2} \hbar^{4} \int d^{D} x \int d^{D} y\left[-\frac{i}{2 \hbar} G_{F}(x-y)\right] \\
\times\left\{\left[-\frac{i}{2 \hbar} G_{F}\left(x-x_{4}\right)\right]\left[-\frac{i}{2 \hbar} G_{F}\left(x-x_{3}\right)\right]\left[-\frac{i}{2 \hbar} G_{F}\left(y-x_{2}\right)\right]\left[-\frac{i}{2 \hbar} G_{F}\left(y-x_{1}\right)\right]\right.
\end{gathered}
$$




$$
\begin{array}{r}
+\left[-\frac{i}{2 \hbar} G_{F}\left(x-x_{4}\right)\right]\left[-\frac{i}{2 \hbar} G_{F}\left(y-x_{3}\right)\right]\left[-\frac{i}{2 \hbar} G_{F}\left(x-x_{2}\right)\right]\left[-\frac{i}{2 \hbar} G_{F}\left(y-x_{1}\right)\right] \\
+\left[-\frac{i}{2 \hbar} G_{F}\left(x-x_{4}\right)\right]\left[-\frac{i}{2 \hbar} G_{F}\left(y-x_{3}\right)\right]\left[-\frac{i}{2 \hbar} G_{F}\left(y-x_{2}\right)\right]\left[-\frac{i}{2 \hbar} G_{F}\left(x-x_{1}\right)\right] \\
+\left[-\frac{i}{2 \hbar} G_{F}\left(y-x_{4}\right)\right]\left[-\frac{i}{2 \hbar} G_{F}\left(y-x_{3}\right)\right]\left[-\frac{i}{2 \hbar} G_{F}\left(x-x_{2}\right)\right]\left[-\frac{i}{2 \hbar} G_{F}\left(x-x_{1}\right)\right] \\
+\left[-\frac{i}{2 \hbar} G_{F}\left(y-x_{4}\right)\right]\left[-\frac{i}{2 \hbar} G_{F}\left(x-x_{3}\right)\right]\left[-\frac{i}{2 \hbar} G_{F}\left(y-x_{2}\right)\right]\left[-\frac{i}{2 \hbar} G_{F}\left(x-x_{1}\right)\right] \\
\left.+\left[-\frac{i}{2 \hbar} G_{F}\left(y-x_{4}\right)\right]\left[-\frac{i}{2 \hbar} G_{F}\left(x-x_{3}\right)\right]\left[-\frac{i}{2 \hbar} G_{F}\left(x-x_{2}\right)\right]\left[-\frac{i}{2 \hbar} G_{F}\left(y-x_{1}\right)\right]\right\} .
\end{array}
$$

The sum of expressions (64) and (66) constitutes the total amplitude which contains the sum of the three channels $s, t$ and $u$. They are expected to be the $p$-adic 4-point amplitudes arising in the limit $p \rightarrow 1$. Thus, in the Fourier space it is expected to be written schematically as

$$
A_{G S}^{(4)}=K_{4}^{G S}+\sum_{i<j} x_{i j}^{G S}
$$

where $x_{i j}^{G S}$ is the propagator $x_{i j}^{G S}=\frac{1}{k_{i} k_{j}+1}$ in momentum space. Then $A_{G S}^{(4)}$ agrees with $\boldsymbol{A}_{\text {top }}^{(4)}(\boldsymbol{k})=-1+\sum_{i<j} x_{i j}^{G S}$ up to the constant $K_{4}^{G S}$, see (54).

In the case of amplitudes of 5-points, the contribution of the term $D \phi^{5}$ in the Lagrangian is written as

$$
\mathcal{Z}[J]=\cdots-D \hbar^{4} \int d^{D} x\left(\frac{\delta}{\delta J(x)}\right)^{5} \mathcal{Z}_{0}[J]+\cdots
$$

Thus, the corresponding 5-point amplitude is

$$
\begin{gathered}
\left.\frac{\delta^{5} \mathcal{Z}[J]}{\delta J\left(x_{1}\right) \delta J\left(x_{2}\right) \delta J\left(x_{3}\right) \delta J\left(x_{4}\right) \delta J\left(x_{5}\right)}\right|_{J=0}=-5 ! D \hbar^{4} \int d^{D} x\left[-\frac{i}{2 \hbar} G_{F}\left(x-x_{1}\right)\right] \\
\times\left[-\frac{i}{2 \hbar} G_{F}\left(x-x_{2}\right)\right]\left[-\frac{i}{2 \hbar} G_{F}\left(x-x_{3}\right)\right]\left[-\frac{i}{2 \hbar} G_{F}\left(x-x_{4}\right)\right]\left[-\frac{i}{2 \hbar} G_{F}\left(x-x_{5}\right)\right] .
\end{gathered}
$$

The contribution will be encoded in the symbol $K_{5}^{G S}$.

Another contribution come from the term $B \phi^{3} \times C^{5} \phi^{4}$. In the Fourier space the corresponding diagrams have precisely 2-vertices, 5 external legs attached to these vertices and one internal leg between the two vertices (see, Section 3 of [14]).

This latter contribution to generating functional is written as

$$
\mathcal{Z}[J]=\cdots-i B C \hbar^{5} \int d^{D} x \int d^{D} y\left(\frac{\delta}{\delta J(x)}\right)^{3}\left(\frac{\delta}{\delta J(y)}\right)^{4} \mathcal{Z}_{0}[J]+\cdots
$$

The 5-point amplitude yields

$$
\begin{gathered}
\left.\frac{\delta^{5} \mathcal{Z}[J]}{\delta J\left(x_{1}\right) \delta J\left(x_{2}\right) \delta J\left(x_{3}\right) \delta J\left(x_{4}\right) \delta J\left(x_{5}\right)}\right|_{J=0} \\
=-i B C(12)^{2} \hbar^{5} \int d^{D} x \int d^{D} y\left[-\frac{i}{2 \hbar} G_{F}(x-y)\right]\left\{\left[-\frac{i}{2 \hbar} G_{F}\left(x-x_{5}\right)\right]\left[-\frac{i}{2 \hbar} G_{F}\left(x-x_{4}\right)\right]\right. \\
\times\left[-\frac{i}{2 \hbar} G_{F}\left(y-x_{3}\right)\right]\left[-\frac{i}{2 \hbar} G_{F}\left(y-x_{2}\right)\right]\left[-\frac{i}{2 \hbar} G_{F}\left(y-x_{1}\right)\right]+\cdots
\end{gathered}
$$




$$
\begin{gathered}
+\left[-\frac{i}{2 \hbar} G_{F}\left(y-x_{5}\right)\right]\left[-\frac{i}{2 \hbar} G_{F}\left(x-x_{4}\right)\right]\left[-\frac{i}{2 \hbar} G_{F}\left(x-x_{3}\right)\right] \\
\left.\times\left[-\frac{i}{2 \hbar} G_{F}\left(y-x_{2}\right)\right]\left[-\frac{i}{2 \hbar} G_{F}\left(y-x_{1}\right)\right]+\cdots\right\} .
\end{gathered}
$$

Moreover, in the computation of the 5-point amplitudes, the last contribution comes from the term

$$
\mathcal{Z}[J]=\cdots+\frac{B^{3} \hbar^{6}}{3 !} \int d^{D} x \int d^{D} y \int d^{D} z\left(\frac{\delta}{\delta J(x)}\right)^{3} \cdot\left(\frac{\delta}{\delta J(y)}\right)^{3} \cdot\left(\frac{\delta}{\delta J(z)}\right)^{3} \mathcal{Z}_{0}[J]+\cdots .
$$

The diagrams associated with this expansion are diagrams with three vertices. Two of them have two external legs and the other leg is internal. The remaining leg has one external line and two internal lines attached.

Then the total contribution is

$$
\begin{gathered}
\left.\frac{\delta^{5} \mathcal{Z}[J]}{\delta J\left(x_{1}\right) \delta J\left(x_{2}\right) \delta J\left(x_{3}\right) \delta J\left(x_{4}\right) \delta J\left(x_{5}\right)}\right|_{J=0}=\frac{B^{3} \hbar^{6} a}{3 !} \int d^{D} x \int d^{D} y \int d^{D} z\left[-\frac{i}{2 \hbar} G_{F}(x-y)\right] \\
\times\left[-\frac{i}{2 \hbar} G_{F}(y-z)\right]\left\{\left[-\frac{i}{2 \hbar} G_{F}\left(y-x_{5}\right)\right]\left[-\frac{i}{2 \hbar} G_{F}\left(z-x_{4}\right)\right]\left[-\frac{i}{2 \hbar} G_{F}\left(z-x_{3}\right)\right]\right. \\
\left.\times\left[-\frac{i}{2 \hbar} G_{F}\left(x-x_{2}\right)\right]\left[-\frac{i}{2 \hbar} G_{F}\left(x-x_{1}\right)\right]+\cdots\right\},
\end{gathered}
$$

where $a$ is a constant.

Summarizing, the 5-point amplitudes $A_{5}$ obtained from Lagrangian (61) can be written schematically (in notation from [14]) by

$$
A_{G S}^{(5)}=K_{5}^{G S}+\sum_{K_{4}^{G S}} x_{i<j}^{G S}+\sum_{\substack{\{i, j, k, l\} \subset\{1,2,3,4,5\} \\ i<j, k<l}} x_{i j}^{G S} x_{k l}^{G S},
$$

where the three terms in the sum corresponds to the contributions of Equations (67), (69) and (71) respectively. Notice that $A_{G S}^{(5)}$ agrees with

$$
\boldsymbol{A}_{\text {top }}^{(5)}(\boldsymbol{k})=2-\sum_{i<j} x_{i j}^{G S}+\sum_{\substack{\{i, j, k, l\} \subset\{1,2,3,4,5\} \\ i<j, k<l}} x_{i j}^{G S} x_{k l}^{G S}
$$

up to the constants $K_{5}^{G S}, K_{4}^{G S}$, see (55).

We now give a precise formulation of Conjecture 2 announced in the Introduction. By using the Feynman rules for the effective Lagrangian given in [14],

$$
A_{\mathbb{Q}_{p}}^{(N)}=K+\sum_{l=2}^{N-3} K_{i_{1} \ldots i_{l}} \sum_{1 \leq a<b \leq l}\left(\frac{p-1}{p} \frac{p^{-k_{i_{a}} k_{i_{b}}-1}}{1-p^{-k_{i_{a}} k_{i_{b}}-1}}\right),
$$

where the constants $K, K_{i_{1} \ldots i_{l}}$ are rational functions in $p$ with rational coefficients. Furthermore, if $N \leq p+1$, the constant term $K$ is not zero. Formally, we have

$$
A_{\text {top }}^{(N)}=\lim _{p \rightarrow 1} A_{\mathbb{Q}_{p}}^{(N)}=K^{t o p}+\sum_{l=2}^{N-3} K_{i_{1} \ldots i_{l}}^{t o p} \sum_{1 \leq a<b \leq l}\left(\frac{1}{k_{i_{a}} k_{i_{b}}+1}\right) .
$$

We denote by $A_{G S}^{(N)}$ the Feynman amplitude of Lagrangian (61). We conjecture that

$$
A_{G S}^{(N)}=K^{G S}+\sum_{l=2}^{N-3} K_{i_{1} \ldots i_{l}}^{G S} \sum_{1 \leq a<b \leq l} x_{i_{a_{a} i_{b}}^{G S}},
$$


where $x_{i_{a} i_{b}}^{G S}=\frac{1}{k_{i_{a}} k_{i_{b}}+1}=\widehat{G_{F}}\left(k_{i_{a}}-k_{i_{b}}\right)$. Notice that we are not asserting the existence of any relation between the constants $K^{\text {top }}, K_{i_{1} \ldots i_{l}}^{\text {top }}$ and the constants $K^{G S}, K_{i_{1} \ldots i_{l}}^{G S}$.

\section{7. $p$-Adic Open String Amplitudes Coupled to a B-Field with Chan-Paton Factors}

Strings propagating in a background with gauge fields was discussed many years ago [57]. In particular, incorporating a Neveu-Schwarz $B$ field in the target space leads to a noncommutative effective gauge theory on the world-volume of D-branes [58]. In [7], the $\mathrm{N}$-point $p$-adic string amplitudes, with Chan-Paton rules and a constant $B$-field (the Ghoshal-Kawano amplitudes) were studied, these amplitudes were introduced in [60]. The study was done by attaching twisted local multivariate zeta functions to the GhoshalKawano amplitudes. In this section we discuss the meromorphic continuation of the Ghoshal-Kawano amplitudes, we also compute the 4 and 5 points amplitudes. Due to the need for a particular symmetry, in this section, we take $p \equiv 3 \bmod 4$.

\subsection{The $p$-Adic Sign Function}

A $p$-adic sign function is a multiplicative character of $\mathbb{Q}_{p}^{\times}$that takes values in $\{ \pm 1\}$. We set $\left[\mathbb{Q}_{p}^{\times}\right]^{2}$ as the multiplicative subgroup of squares in $\mathbb{Q}_{p}^{\times}$, i.e.

$$
\left[\mathbb{Q}_{p}^{\times}\right]^{2}=\left\{a \in \mathbb{Q}_{p} ; a=b^{2} \text { for some } b \in \mathbb{Q}_{p}^{\times}\right\} .
$$

Let $\varepsilon \in\{1, \ldots, p-1\}$ such that $\left(\frac{\varepsilon}{p}\right)=-1$, where $(\div)$ is the Legendre symbol (see for instance the Appendix of [7]). For $p \neq 2$ we have

$$
\mathbb{Q}_{p}^{\times} /\left[\mathbb{Q}_{p}^{\times}\right]^{2}=\{1, \varepsilon, p, \varepsilon p\},
$$

this means that any nonzero $p$-adic number can be written uniquely as

$$
x=\tau a^{2}, \text { with } a \in \mathbb{Q}_{p}^{\times} \text {and } \tau \in \mathbb{Q}_{p}^{\times} /\left[\mathbb{Q}_{p}^{\times}\right]^{2} .
$$

For a fixed $\tau \in\{\varepsilon, p, \varepsilon p\}$, and $x \in \mathbb{Q}_{p}^{\times}$, we define the $p$-adic sign function

$$
\operatorname{sgn}_{\tau}(x):= \begin{cases}1 & \text { if } x=a^{2}-\tau b^{2} \text { for } a, b \in \mathbb{Q}_{p} \\ -1 & \text { otherwise. }\end{cases}
$$

The following is the list of all the possible $p$-adic sign functions:

\begin{tabular}{cc}
\hline $\mathbf{p} \equiv \mathbf{1} \bmod \mathbf{4}$ & $\mathbf{p} \equiv \mathbf{3} \bmod \mathbf{4}$ \\
\hline $\operatorname{sgn}_{\varepsilon}(x)=(-1)^{\operatorname{ord}(x)}$ & $\operatorname{sgn}_{\varepsilon}(x)=(-1)^{\operatorname{ord}(x)}$ \\
\hline $\operatorname{sgn}_{p}(x)=\left(\frac{x_{0}}{p}\right)$ & $\operatorname{sgn}_{p}(x)=(-1)^{\operatorname{ord}(x)}\left(\frac{x_{0}}{p}\right)$ \\
\hline $\operatorname{sgn}_{\varepsilon p}(x)=(-1)^{\operatorname{ord}(x)}\left(\frac{x_{0}}{p}\right)$ & $\operatorname{sgn}_{\varepsilon p}(x)=\left(\frac{x_{0}}{p}\right)$, \\
\hline
\end{tabular}

see [68]. The function $\operatorname{sgn}_{\tau}$ is a multiplicative character, which means that

$$
\operatorname{sgn}_{\tau}(x y)=\operatorname{sgn}_{\tau}(x) \operatorname{sgn}_{\tau}(y) .
$$

Additionally $\operatorname{sgn}_{\tau}$ is a locally constant function in $\mathbb{Q}_{p}^{\times}$, which means that $\operatorname{sgn}_{\tau}(x-y)=$ $\operatorname{sgn}_{\tau}(x)$ if $|y|_{p}<|x|_{p}$.

We need the symmetry $\operatorname{sgn}_{\tau}(-y)=-\operatorname{sgn}_{\tau}(y)$ (i.e. $\operatorname{sgn}_{\tau}(-1)=-1$ ), this requires $p \equiv 3 \bmod 4$ and $\tau \in\{p, \varepsilon p\}$. Finally, for any $x \in \mathbb{Q}_{p}^{\times}$, we define the $p$-adic Heaviside step function as 


$$
H_{\tau}(x)=\frac{1}{2}\left(1+\operatorname{sgn}_{\tau}(x)\right)= \begin{cases}1 & \text { if } \operatorname{sgn}_{\tau}(x)=1 \\ 0 & \text { if } \operatorname{sgn}_{\tau}(x)=-1 .\end{cases}
$$

\subsection{A Class of Twisted Multivariate Local Zeta Functions}

Let

$$
f:=\left(f_{1}, \ldots, f_{m}\right), x:=\left(\chi_{1}, \ldots, \chi_{m}\right)
$$

be vectors of non-constant polynomials and multiplicative characters, respectively. And $s:=$ $\left(s_{1}, \ldots, s_{m}\right) \in \mathbb{C}^{m}$. The twisted multivariate local zeta functions have the form:

$$
Z_{\Theta}(s, \chi, f)=\int_{\mathbb{Q}_{p}^{n} \backslash \cup_{i=1}^{m} f^{-1}(0)} \Theta(x) \prod_{i=1}^{m} \chi_{i}\left(a c\left(f_{i}(x)\right)\right)\left|f_{i}(x)\right|_{p}^{s_{i}} \prod_{i=1}^{n} d x_{i},
$$

with $\operatorname{Re}\left(s_{i}\right)>0$ for all $i, a c$ stands for the angular component and $\Theta(x)$ is a test function. Integrals of type (74) are holomorphic functions in $s$, which admit meromorphic continuations as rational functions in the variables $p^{-s_{1}}, \ldots, p^{-s_{m}}$ to the whole $\mathbb{C}^{m}$, Théorème 1.1.4. of [36], see also [26]. The case when $\chi_{i}$ is the trivial character has been studied previously, see e.g., Lemma 8.2.1 of [26]. The case when $\chi_{i}=\operatorname{sgn}_{\tau}$ is new.

Using Hironaka's resolution of singularities theorem [50], in [7] we show that $Z_{\Theta}(s, \chi, f)$ admits a meromorphic continuation as a rational function in the variables $p^{-s_{1}}, \ldots, p^{-s_{m}}$. More precisely,

$$
Z_{\Theta}(s, \chi, f)=\frac{L_{\Theta, \chi}(s)}{\prod_{j \in \mathcal{T}}\left(1-p^{-\sum_{i=1}^{m} N_{i, j} s_{j}-v_{j}}\right)},
$$

where $L_{\Theta, \chi}(s)$ is a polynomial in the variables $p^{-s_{1}}, \ldots, p^{-s_{m}}$, and the real parts of its poles belong to the finite union of hyperplanes

$$
\sum_{i=1}^{m} N_{i, j} \operatorname{Re}\left(s_{i}\right)+v_{j}=0, \quad \text { for } j \in \mathcal{T} .
$$

This result is a variation of Théorème 1.1.4. in [36].

\subsection{The Ghoshal-Kawano Local Zeta Function}

In [60] Ghoshal and Kawano proposed the following amplitude (for the $N$-point tree-level, $p$-adic open string amplitude, with Chan-Paton rules in a constant $B$-field):

$$
\begin{aligned}
& A^{(N)}\left(\boldsymbol{k}, \theta, \tau ; x_{1}, x_{N-1}\right):=\int_{\mathbb{Q}_{p}^{N-3} \backslash \Lambda} \prod_{i=2}^{N-2}\left|x_{i}\right|_{p}^{k_{1} k_{i}}\left|1-x_{i}\right|_{p}^{k_{N-1} \boldsymbol{k}_{i}} H_{\tau}\left(x_{i}\right) H_{\tau}\left(1-x_{i}\right) \\
& \quad \times \prod_{2 \leq i<j \leq N-2}\left|x_{i}-x_{j}\right|_{p}^{k_{i} \boldsymbol{k}_{j}} H_{\tau}\left(x_{i}-x_{j}\right) \\
& \quad \times \exp \left\{-\frac{\sqrt{-1}}{2}\left(\sum_{1 \leq i<j \leq N-1}\left(\boldsymbol{k}_{i} \theta \boldsymbol{k}_{j}\right) \operatorname{sgn}_{\tau}\left(x_{i}-x_{j}\right)\right)\right\} \prod_{i=2}^{N-2} d x_{i},
\end{aligned}
$$

where $N \geq 4, \boldsymbol{k}=\left(\boldsymbol{k}_{1}, \ldots, \boldsymbol{k}_{N}\right), \boldsymbol{k}_{i}=\left(k_{0, i}, \ldots, k_{l, i}\right), i=1, \ldots, N$, is the momentum vector of the $i$-th tachyon (with Minkowski product $\boldsymbol{k}_{i} \boldsymbol{k}_{j}=-k_{0, i} k_{0, j}+k_{1, i} k_{1, j}+\cdots+k_{l, i} k_{l, j}$ ), $\theta$ is a fixed antisymmetric bilinear form that is built with the inverse of the $B$-field; $\prod_{i=2}^{N-2} d x_{i}$ is the normalized Haar measure of $\mathbb{Q}_{p}^{N-3}$, 


$$
\Lambda:=\left\{\left(x_{2}, \ldots, x_{N-2}\right) \in \mathbb{Q}_{p}^{N-3} ; \prod_{i=2}^{N-2} x_{i}\left(1-x_{i}\right) \prod_{2 \leq i<j \leq N-2}\left(x_{i}-x_{j}\right)=0\right\},
$$

and the momentum vectors obey

$$
\sum_{i=1}^{N} \boldsymbol{k}_{i}=\mathbf{0}, \quad \boldsymbol{k}_{i} \boldsymbol{k}_{i}=2 \text { for } i=1, \ldots, N
$$

To preserve the symmetry under the exchange of external momentum vectors, we require that $\operatorname{sgn}_{\tau}\left(x_{i}-x_{j}\right)=-\operatorname{sgn}_{\tau}\left(x_{j}-x_{i}\right)$, or equivalently $\operatorname{sgn}_{\tau}(-1)=-1$. Then for the rest of this section we assume that $\tau \in\{p, \varepsilon p\}$.

To simplify the notation, we introduce the variables $s_{i j} \in \mathbb{C}$, and $\tilde{s}_{i j} \in \mathbb{R}$ for $1 \leq i<j \leq N-1$. We also set

$$
\begin{aligned}
F(x, s, \tau):=\prod_{i=2}^{N-2}\left|x_{i}\right|_{p}^{s_{1 i}}\left|1-x_{i}\right|_{p}^{s_{(N-1) i}} H_{\tau}\left(x_{i}\right) H_{\tau}\left(1-x_{i}\right) & \times \prod_{2 \leq i<j \leq N-2}\left|x_{i}-x_{j}\right|_{p}^{s_{i j}} H_{\tau}\left(x_{i}-x_{j}\right),
\end{aligned}
$$

and

$$
E\left(x, \widetilde{s}, \tau ; x_{1}, x_{N-1}\right):=\exp \left\{\frac{-\sqrt{-1}}{2}\left(\sum_{1 \leq i<j \leq N-1} \widetilde{s}_{i j} \operatorname{sgn}_{\tau}\left(x_{1}-x_{j}\right)\right)\right\} .
$$

Now, we define the Ghoshal-Kawano local zeta function as

$$
Z^{(N)}\left(\boldsymbol{s}, \widetilde{\boldsymbol{s}}, \tau ; x_{1}, x_{N-1}\right)=\int_{\mathbb{Q}_{p}^{N-3} \backslash \Lambda} F(\boldsymbol{x}, \boldsymbol{s}, \tau) E\left(\boldsymbol{x}, \widetilde{\boldsymbol{s}}, \tau ; x_{1}, x_{N-1}\right) \prod_{i=2}^{N-2} d x_{i} .
$$

For the sake of simplicity, we use $\mathbb{Q}_{p}^{N-3}$ as domain of integration in (79) from now on. We now consider the convergence of the amplitudes (76). By using that $\left|E\left(\boldsymbol{x}, \widetilde{\boldsymbol{s}}, \tau ; x_{1}, x_{N-1}\right)\right|=1$, $\left|H_{\tau}\left(x_{i}\right)\right| \leq 1,\left|H_{\tau}\left(1-x_{i}\right)\right| \leq 1$, for any $i$, and that $\left|H_{\tau}\left(x_{i}-x_{j}\right)\right| \leq 1$, for any $i, j$, we have

$$
\begin{aligned}
&\left|Z^{(N)}\left(s, \widetilde{s}, \tau ; x_{1}, x_{N-1}\right)\right| \\
& \leq \int_{\mathbb{Q}_{p}^{N-3}} \prod_{i=2}^{N-2}\left|x_{i}\right|_{p}^{\operatorname{Re}\left(s_{1 i}\right)}\left|1-x_{i}\right|_{p}^{\operatorname{Re}\left(s_{(N-1) i}\right)} \prod_{2 \leq i<j \leq N-2}\left|x_{i}-x_{j}\right|_{p}^{\operatorname{Re}\left(s_{i j}\right)} \prod_{i=2}^{N-2} d x_{i} \\
&=Z^{(N)}(\operatorname{Re}(s)),
\end{aligned}
$$

where $Z^{(N)}(s)$ is the Koba-Nielsen string amplitude studied in [6,8]. By applying the results of [6,8], integral $Z^{(N)}(\operatorname{Re}(s))$ is holomorphic in an open set $\mathcal{K} \subset \mathbb{C}^{D}$, therefore

$$
Z^{(N)}(s, \widetilde{s}, \tau) \text { is holomorphic in } s \in \mathcal{K} \text { for any } \widetilde{s}, \tau, x_{1}, x_{N-1} \text {. }
$$

It is important to notice that if any of the integration variables is in $\mathbb{Q}_{p} \backslash \mathbb{Z}_{p}$, then due to the local constancy of $\operatorname{sgn}_{\tau}$, the factor $H_{\tau}(x) H_{\tau}(-x)$ appears in $F(x, s, \tau)$, and $H_{\tau}(x) H_{\tau}(-x)=$ 0 . For this reason, we redefine the Ghoshal-Kawano local zeta function as

$$
Z^{(N)}\left(s, \widetilde{s}, \tau ; x_{1}, x_{N-1}\right):=\int_{\mathbb{Z}_{p}^{N-3}} F(\boldsymbol{x}, \boldsymbol{s}, \tau) E\left(\boldsymbol{x}, \widetilde{\boldsymbol{s}}, \tau ; x_{1}, x_{N-1}\right) \prod_{i=2}^{N-2} d x_{i}
$$




\subsection{Ghoshal-Kawano Amplitude as a Local Zeta Function}

For $\tilde{s} \in \mathbb{R}$ we have

$$
\exp \left\{\frac{-\sqrt{-1} \widetilde{s}}{2} \operatorname{sgn}_{\tau}(x)\right\}=\cos \left(\frac{\widetilde{s}}{2}\right)-\sqrt{-1} \operatorname{sgn}_{\tau}(x) \sin \left(\frac{\widetilde{s}}{2}\right) .
$$

Using this identity and the convention $x_{1}=0, x_{N-1}=1$, we have

$$
E(\boldsymbol{x}, \widetilde{\boldsymbol{s}}, \tau)=\sum_{I, J, K} E_{I, J, K}(\widetilde{\boldsymbol{s}}) \prod_{j \in I} \operatorname{sgn}_{\tau}\left(x_{j}\right) \prod_{j \in J} \operatorname{sgn}_{\tau}\left(1-x_{j}\right) \prod_{i, j \in K} \operatorname{sgn}_{\tau}\left(x_{i}-x_{j}\right) .
$$

In a similar way, we obtain that

$$
\begin{aligned}
& \prod_{i=2}^{N-2} H_{\tau}\left(x_{i}\right) H_{\tau}\left(1-x_{i}\right) \prod_{2 \leq i<j \leq N-2} H_{\tau}\left(x_{i}-x_{j}\right) \\
& =\sum_{I, J, K} e_{I, J, K} \prod_{j \in I} \operatorname{sgn}_{\tau}\left(x_{j}\right) \prod_{j \in J} \operatorname{sgn}_{\tau}\left(1-x_{j}\right) \prod_{i, j \in K} \operatorname{sgn}_{\tau}\left(x_{i}-x_{j}\right),
\end{aligned}
$$

Using Formulae (82) and (83), and assuming $x_{1}=0, x_{N-1}=1, x_{N}=\infty$, we can write the Ghoshal-Kawano zeta function as a finite sum of integrals of type

$$
\begin{aligned}
C(\widetilde{\mathbf{s}}) \int_{\mathbb{Z}_{p}^{N-3}} \prod_{i=2}^{N-2}\left|x_{i}\right|_{p}^{s_{1 i}}\left|1-x_{i}\right|_{p}^{s_{(N-1) i}} \prod_{2 \leq i<j \leq N-2}\left|x_{i}-x_{j}\right|_{p}^{s_{i j}} \prod_{j \in I} \chi_{\tau}\left(x_{j}\right) \prod_{j \in J} \chi_{\tau}\left(1-x_{j}\right) & \\
& \times \prod_{i, j \in K} \chi_{\tau}\left(x_{i}-x_{j}\right) \prod_{i=2}^{N-2} d x_{i},
\end{aligned}
$$

where $C(\widetilde{\boldsymbol{s}})$ is an $\mathbb{R}$-analytic function and $\chi_{\tau}$ is a trivial character or $\operatorname{sgn}_{\tau}$. This expression is a local zeta functions of the type (74), which implies that amplitudes (76) are equal to a finite sum of local zeta functions and consequently they admit a meromorphic continuation in the kinematic parameters. Finally, we point out that the meromorphic continuation of the Ghoshal-Kawano local zeta function (80) is also valid without taking the normalization $x_{1}=0, x_{N-1}=1, x_{N}=\infty$, see Section V-C of [7].

\subsection{Explicit Computation of $Z^{(4)}(s, \widetilde{s}, \tau)$}

We first notice that the exponential factor $E^{(4)}(\tilde{s}, \tau)$ can be taken outside the integral, i.e., the four-point Ghoshal-Kawano zeta function is

$$
\begin{aligned}
& Z^{(4)}(\boldsymbol{s}, \widetilde{\mathbf{s}}, \tau)=\exp \left\{\frac{i}{2}\left(\widetilde{s}_{13}+\widetilde{s}_{12}+\widetilde{s}_{23}\right)\right\} \\
& \quad \times \int_{\mathbb{Z}_{p}}\left|x_{2}\right|_{p}^{s_{12}}\left|1-x_{2}\right|_{p}^{s_{32}} H_{\tau}\left(x_{2}\right) H_{\tau}\left(1-x_{2}\right) d x_{2} .
\end{aligned}
$$

The computation of this integral is based on the calculation of the following integrals: assume that $S \subset \mathbb{Z}_{p} \backslash\{0\}$ satisfies $-S=S$, then

$$
\int_{S}\left|x_{2}\right|_{p}^{s_{12}} \operatorname{sgn}_{\tau}\left(x_{2}\right) d x_{2}=0, \text { for } \tau \in\{p, \varepsilon p\},
$$

and

$$
\begin{aligned}
I(s, \tau) & =\int_{\mathbb{Z}_{p}}\left|x_{2}\right|_{p}^{s_{12}}\left|1-x_{2}\right|_{p}^{s_{32}} H_{\tau}\left(x_{2}\right) H_{\tau}\left(1-x_{2}\right) d x_{2} \\
& =\frac{p-3}{4 p}+\frac{p^{-1-s_{12}}\left(1-p^{-1}\right)}{2\left(1-p^{-1-s_{12}}\right)}+\frac{p^{-1-s_{32}}\left(1-p^{-1}\right)}{2\left(1-p^{-1-s_{32}}\right)} .
\end{aligned}
$$


For further details, the reader may consult [7]. The explicit expression for the 4-point amplitude, also reported in [60], is

$$
\begin{aligned}
Z^{(4)}(\boldsymbol{s}, \widetilde{\mathbf{s}}, \tau)=\exp \left\{\frac { i } { 2 } \left(\widetilde{s}_{13}+\widetilde{s}_{12}\right.\right. & \left.\left.+\widetilde{s}_{23}\right)\right\} \\
& \times\left(\frac{p-3}{4 p}+\frac{p^{-1-s_{12}}\left(1-p^{-1}\right)}{2\left(1-p^{-1-s_{12}}\right)}+\frac{p^{-1-s_{32}}\left(1-p^{-1}\right)}{2\left(1-p^{-1-s_{32}}\right)}\right)
\end{aligned}
$$

which is holomorphic in

$$
\operatorname{Re}\left(s_{12}\right)>-1 \text { and } \operatorname{Re}\left(s_{32}\right)>-1 .
$$

7.6. Explicit Computation of $Z^{(5)}(s, \widetilde{s}, \tau)$

After some simple considerations involving sign functions,

$$
Z^{(5)}(\boldsymbol{s}, \widetilde{\boldsymbol{s}}, \boldsymbol{\tau})=E^{(5)}(\widetilde{\boldsymbol{s}}) L(\boldsymbol{s}, \tau):=E^{(5)}(\widetilde{\boldsymbol{s}}) \int_{\mathbb{Z}_{p}^{2}} F^{(5)}\left(x_{2}, x_{3}, \boldsymbol{s}, \tau\right) d x_{2} d x_{3} .
$$

where

$$
\begin{aligned}
F^{(5)}\left(x_{2}, x_{3}, s, \tau\right) & =\left|x_{2}\right|_{p}^{s_{12}}\left|x_{3}\right|_{p}^{s_{13}}\left|1-x_{2}\right|_{p}^{s_{42}}\left|1-x_{3}\right|_{p}^{s_{43}}\left|x_{2}-x_{3}\right|_{p}^{s_{23}} \\
& \times H_{\tau}\left(x_{2}\right) H_{\tau}\left(x_{3}\right) H_{\tau}\left(1-x_{2}\right) H_{\tau}\left(1-x_{3}\right) H_{\tau}\left(x_{2}-x_{3}\right) .
\end{aligned}
$$

and

$$
E^{(5)}(\widetilde{s})=\exp \left\{\frac{\sqrt{-1}}{2}\left(\widetilde{s}_{14}+\widetilde{s}_{12}+\widetilde{s}_{13}+\widetilde{s}_{24}+\widetilde{s}_{34}+\widetilde{s}_{32}\right)\right\},
$$

see [7] for further details. To compute the integral $L(s, \tau)$, we subdivide the domain of integration as

$$
\mathbb{Z}_{p}^{2}=\bigsqcup_{i, j=0}^{p-1}\left(i+p \mathbb{Z}_{p}\right) \times\left(j+p \mathbb{Z}_{p}\right),
$$

then $L(s, \tau)$ becomes

$$
L(s, \tau)=\sum_{i, j=0}^{p-1} L_{i j}(s, \tau)=: \sum_{i, j=0}^{p-1} \int_{i+p \mathbb{Z}_{p} \times j+p \mathbb{Z}_{p}} F^{(5)}\left(x_{2}, x_{3}, s, \tau\right) d x_{2} d x_{3} .
$$

The computation of the integrals $L_{i j}(s, \tau)$ is a simple but technical calculation, see [7] for further details. The explicit expression for the 5-point Ghoshal-Kawano zeta function is

$$
\begin{gathered}
Z^{(5)}(s, \widetilde{\mathbf{s}})=E^{(5)}(\widetilde{\mathbf{s}})\left\{\frac{(p-3)(p-7)}{32 p^{2}}+\left(\frac{p-3}{8 p}\right)\left[\frac{p^{-1-s_{23}\left(1-p^{-1}\right)}}{\left(1-p^{-1-s_{23}}\right)}\right.\right. \\
\left.+\frac{p^{-1-s_{13}}\left(1-p^{-1}\right)}{\left(1-p^{-1-s_{13}}\right)}+\frac{p^{-1-s_{42}}\left(1-p^{-1}\right)}{\left(1-p^{-1-s_{42}}\right)}\right]+\frac{\left(1-p^{-1}\right)^{2}}{4} \frac{p^{-2-s_{13}-s_{42}}}{\left(1-p^{-1-s_{13}}\right)\left(1-p^{\left.-1-s_{42}\right)}\right.} \\
+\frac{1}{4} \frac{p^{-s_{12}-s_{13}-s_{23}-2}\left(1-p^{-1}\right)}{1-p^{-s_{12}-s_{13}-s_{23}-2}}\left[\frac{1}{2}-\frac{3}{2 p}+\frac{p^{-1-s_{23}}\left(1-p^{-1}\right)}{1-p^{-1-s_{23}}}+\frac{p^{-1-s_{13}}\left(1-p^{-1}\right)}{1-p^{-1-s_{13}}}\right] \\
\left.+\frac{1}{4} \frac{p^{-s_{42}-s_{43}-s_{23}-2}\left(1-p^{-1}\right)}{1-p^{-s_{42}-s_{43}-s_{23}-2}}\left[\frac{1}{2}-\frac{3}{2 p}+\frac{p^{-1-s_{23}}\left(1-p^{-1}\right)}{1-p^{-1-s_{23}}}+\frac{p^{-1-s_{42}}\left(1-p^{-1}\right)}{1-p^{-1-s_{42}}}\right]\right\} .
\end{gathered}
$$


This function is holomorphic in

$$
\begin{aligned}
& \operatorname{Re}\left(s_{13}\right)>-1 ; \quad \operatorname{Re}\left(s_{23}\right)>-1 ; \quad \operatorname{Re}\left(s_{42}\right)>-1 ; \\
& \operatorname{Re}\left(s_{12}+s_{13}+s_{23}\right)>-2 ; \quad \operatorname{Re}\left(s_{42}+s_{43}+s_{23}\right)>-2 .
\end{aligned}
$$

\section{Resolution of Singularities and Multivariate Igusa Zeta Functions}

\subsection{Local Fields}

We take $\mathbb{K}$ to be a non-discrete locally compact field of characteristic zero. Then $\mathbb{K}$ is $\mathbb{R}, \mathbb{C}$, or a finite extension of $\mathbb{Q}_{p}$, the field of $p$-adic numbers. If $\mathbb{K}$ is $\mathbb{R}$ or $\mathbb{C}$, we say that $\mathbb{K}$ is an $\mathbb{R}$-field, otherwise we say that $\mathbb{K}$ is a $p$-field.

For $a \in \mathbb{K}$, we define the modulus $|a|_{\mathbb{K}}$ of $a$ by

$$
|a|_{\mathbb{K}}=\left\{\begin{array}{l}
\text { the rate of change of the Haar measure in }(\mathbb{K},+) \text { under } x \rightarrow a x \\
\text { for } a \neq 0, \\
0 \text { for } a=0 .
\end{array}\right.
$$

is well-known that if $\mathbb{K}$ is an $\mathbb{R}$-field, then $|a|_{\mathbb{R}}=|a|$ and $|a|_{\mathbb{C}}=|a|^{2}$, where $|\cdot|$ denotes the usual absolute value in $\mathbb{R}$ or $\mathbb{C}$, and, if $\mathbb{K}$ is a $p$-field, then $|\cdot|_{\mathbb{K}}$ is the normalized absolute value in $\mathbb{K}$.

\section{2. $\mathbb{K}$-Analytic Manifolds and Resolution of Singularities}

We review the basic definitions of $\mathbb{K}$-analytic manifolds following Igusa's book [26].

Let $\mathbb{K}$ be a local field of characteristic zero, and let $V$ be a nonempty open subset of $\mathbb{K}^{n}$ and let $f: V \rightarrow \mathbb{K}$ be a function. We say that $f$ is a $\mathbb{K}$-analytic function on $V$, if for every point $a=\left(a_{1}, \ldots, a_{n}\right) \in V$, there exists an element $f_{a}(x)$ of $\mathbb{K}\langle\langle x-a\rangle\rangle=\mathbb{K}\left\langle\left\langle x_{1}-\right.\right.$ $\left.\left.a_{1}, \ldots, x_{n}-a_{n}\right\rangle\right\rangle$ (the ring of convergent power series around $a$ ) such that $f(x)=f_{a}(x)$ for any point $x$ near $a$. A map $g=\left(g_{1}, \ldots, g_{m}\right): V \rightarrow \mathbb{K}^{m}$ is called $\mathbb{K}$ - analytic mapping on $V$ if each $g_{i}$ is an analytic function on $V$. Let $X$ Hausdorff space and let $n$ be a fixed nonnegative. Let $U$ be a nonempty open subset of $X$, if $\phi_{U}: U \rightarrow \phi_{U}(U)$ is a homeomorphism, where $\phi_{U}(U)$ is a nonempty open subset of $\mathbb{K}^{n}$, then we say that the pair $\left(U, \phi_{U}(U)\right)$ is a chart. For a variable point $x \in U, \phi(x)=\left(x_{1}, \ldots, x_{n}\right)$ are called the local coordinates of $x$. A collection of charts $\left\{U, \phi_{U}\right\}$ is called an atlas of $X$ if the union of all open sets $V$ is $X$ and for every $U, U^{\prime}$ such that $U \cap U^{\prime} \neq \varnothing$ the map

$$
\phi_{U^{\prime}} \circ \phi_{U}^{-1}: \phi_{U}\left(U \cap U^{\prime}\right) \rightarrow \phi_{U^{\prime}}\left(U \cap U^{\prime}\right)
$$

is $\mathbb{K}$ - analytic. There is an equivalence relation over the set of atlases on $X$. Two atlases are equivalent if their union is also an atlas. Thus, any equivalence class is called an $n$-dimensional K-analytic structure on $X$. Hence, we say that $X$ is a $\mathbb{K}$-analytic manifold and we write $\operatorname{dim}(X)=n$.

Suppose that $X$ and $Y$ are two $\mathbb{K}$ - analytical manifolds defined by the atlases $\left\{\left(U, \phi_{U}(U)\right)\right\}$ and $\left\{\left(V, \psi_{V}(V)\right)\right\}$, respectively, and $f: X \rightarrow Y$ a map. If for every $U, V$ such that $U \cap f^{-1}(V) \neq \varnothing$, the map

$$
\psi_{V} \circ f \circ \phi_{U}^{-1}: \phi_{U}\left(U \cap f^{-1}(V)\right) \rightarrow \mathbb{K}^{\operatorname{dim}(Y)}
$$

is $\mathbb{K}$-analytic, then $f$ is called a $K$-analytic map. If $Y=\mathbb{K}$ we say that $f$ is a $\mathbb{K}$-analytic function on $X$. This definition does not depend on the choice of atlases into the equivalence class.

Let $X$ be a $\mathbb{K}$-analytic manifold defined by an atlas $\left\{\left(U, \phi_{U}\right)\right\}$ and let $Y$ be a nonempty open set of $X$. If $U^{\prime}=U \cap Y \neq \varnothing$ we put $\phi_{U^{\prime}}=\phi_{U^{\prime}}=\left.\phi_{U}\right|_{U^{\prime}}$. Then $\left\{\left(U^{\prime}, \phi_{U^{\prime}}\right)\right\}$ is an atlas for $Y$. Hence, $Y$ is an open $\mathbb{K}$ - analytic submanifold of $X$. Furthermore, $\operatorname{dim}(Y)=\operatorname{dim}(X)$. 
Now let $Y$ be a nonempty closed subset of a $\mathbb{K}$-analytic manifold $X$ of dimension $n$. Suppose that $X$ is defined by an atlas $\left\{\left(U, \phi_{U}\right)\right\}$ with the following property: If $\phi_{U}(x)=$ $\left(x_{1}, \ldots, x_{n}\right)$ and $U^{\prime}=Y \cap U \neq \varnothing$, there exist $F_{1}, \ldots, F_{m} \mathbb{K}$-analytic functions on $U$ with $0<m \leq n$ such that $U^{\prime}$ becomes the set of all $x$ in $U$ such that $F_{1}(x)=\ldots=F_{m}(x)=0$ and

$$
\operatorname{det}\left[\frac{\partial F_{i}}{\partial x_{j}}\right]_{\substack{1 \leq i \leq m \\ 1 \leq j \leq m}}(a) \neq 0,
$$

for every $a \in U^{\prime}$. By the implicit function theorem, the mapping

$$
x \mapsto\left(F_{1}(x), \ldots, F_{m}(x), x_{m+1}, \ldots, x_{n}\right)
$$

gives a $\mathbb{K}$-bianalytic map from a neighborhood of $a$ in $U$ to its image in $K^{n}$. If we denote by $V$ the intersection of such neighborhood and $Y$, and put $\psi_{V}(x)=\left(x_{m+1}, \ldots, x_{n}\right)$ for every $x \in V$. Then $\left(V, \psi_{V}\right)$ for all $V$ and for each $U$ as above, gives an atlas on $Y$. Thus, $Y$ becomes a $\mathbb{K}$-analytic closed submanifold of $X$ of dimension $n-m$.

Assume that $U$ and $V$ are nonempty open sets that contain a point $a$ of $X$, and let $f, g$ be two $\mathbb{K}$-analytic functions respectively on $U, V$ such that $\left.f\right|_{W}=\left.g\right|_{W}$ for some nonempty open set $W$ such that $a \in W \subseteq U \cap V$. Then we say that $f$ and $g$ are equivalent. An equivalence class is called a germ of analytic function at $a$. The set of such equivalence classes becomes a local ring $\mathcal{O}_{X, a}$ with maximal ideal $m_{X, a}=\left\{f \in \mathcal{O}_{X, a}: f(a)=0\right\}$. In addition, $\mathbb{K} \subseteq \mathcal{O}_{X, a}$.

\section{Multivariate Igusa Zeta Functions}

Let $\mathbb{K}$ be a local field. If $\mathbb{K}$ is a $p$-field, resp. an $\mathbb{R}$-field, we denote by $\mathcal{D}\left(\mathbb{K}^{n}\right)$ the $\mathbb{C}$-vector space consisting of all $\mathbb{C}$-valued locally constant functions, resp. all smooth functions, on $\mathbb{K}^{n}$, with compact support. An element of $\mathcal{D}\left(\mathbb{K}^{n}\right)$ is called a test function.

Let $f_{i}(\boldsymbol{x}) \in \mathbb{K}\left[x_{1}, \ldots, x_{n}\right]$ be a non-constant polynomial for $i=1, \ldots, m$. We set $f=\left(f_{1}, \ldots, f_{m}\right)$ and $s=\left(s_{1}, \ldots, s_{m}\right) \in \mathbb{C}^{m}$. Let $\Phi$ be a test function. The multivariate local zeta function attached to $(f, \Phi)$ is defined as

$$
Z_{\Phi}(f, s)=\int_{\mathbb{K}^{n} \backslash D_{\mathbb{K}}} \Phi(x) \prod_{i=1}^{m}\left|f_{i}(x)\right|_{\mathbb{K}}^{s_{i}} \prod_{i=1}^{n} d x_{i}, \quad \text { when } \operatorname{Re}\left(s_{i}\right)>0 \text { for all } i .
$$

where $D_{\mathbb{K}}:=\cup_{i=1}^{m} f_{i}^{-1}(0)$ is the divisor attached to $f_{i}(x), i=1, \ldots, m$. In the multivariate case i.e., for $m \geq 1$, the local zeta functions over local fields of zero characteristic were studied by Loeser [36]. In the case of zero characteristic, the main tool to show the existence of a meromorphic continuation of the multivariate local function is the Hironaka's resolution of singularities theorem. By applying this theorem to the divisor $D_{\mathbb{K}}$, the mutivariate local zeta function is reduced to the case of monomial integrals $[25,26,36]$. Currently, the methods used by Igusa are not available in positive characteristic, so the problem of the meromorphic continuations in this setting it is still an open problem.

Theorem 2 (Hironaka, [50]). Let $\mathbb{K}$ be a local field of characteristic zero. There exists an embedded resolution $\sigma: X \rightarrow \mathbb{K}^{n}$ of $D_{\mathbb{K}}$, i.e.,

(i) $X$ is an $n$-dimensional $\mathbb{K}$-analytic manifold, $\sigma$ is a proper $\mathbb{K}$-analytic map which is a composition of a finite number of blow-ups at closed submanifolds, and which is an isomorphism outside of $\sigma^{-1}\left(D_{\mathbb{K}}\right)$;

(ii) $\sigma^{-1}\left(D_{\mathbb{K}}\right)$ is a normal crossings divisor, meaning that $\sigma^{-1}\left(D_{\mathbb{K}}\right)=\cup_{i \in T} E_{i}$, where the $E_{i}$ are closed submanifolds of $X$ of codimension one, each equipped with an m-tuple of non-negative integers $\left(N_{f_{1}, i}, \ldots, N_{f_{m}, i}\right)$ and a positive integer $v_{i}$, satisfying the following. At every point $b$ of $X$ 
there exist local coordinates $\left(y_{1}, \ldots, y_{n}\right)$ on $X$ around $b$ such that if $E_{1}, \ldots, E_{r}$ are the $E_{i}$ containing $b$, we have on some open neighborhood $V$ of $b$ that $E_{i}$ is given by $y_{i}=0$ for $i \in\{1, \ldots, r\}$,

$$
\sigma^{*}\left(d x_{1} \wedge \ldots \wedge d x_{n}\right)=\eta(y)\left(\prod_{i=1}^{r} y_{i}^{v_{i}-1}\right) d y_{1} \wedge \ldots \wedge d y_{n}
$$

and

$$
f_{j}^{*}(y):=\left(f_{j} \circ \sigma\right)(y)=\varepsilon_{f_{j}}(y) \prod_{i=1}^{r} y_{i}^{N_{f_{j}, i}}, \quad \text { for } j=1, \ldots, m,
$$

where $\eta(y)$ and the $\varepsilon_{f_{j}}(y)$ belong to $\mathcal{O}_{X, b}^{\times}$, the group of units of the local ring of $X$ at $b$.

The Hironaka resolution theorem allows expressing a multivariate local zeta function as a linear combination of monomial integrals, through a finite sequence of changes of variables. We have the following theorem on multivariate local zeta functions over local fields of zero characteristic.

Theorem 3. (Lemma 6.4, Remark 2 of [6]) Let $f_{1}(x), \ldots, f_{m}(x) \in \mathbb{K}\left[x_{1}, \ldots, x_{n}\right]$ be nonconstant polynomials and $\Phi: \mathbb{K}^{n} \rightarrow \mathbb{C}$ a smooth function with compact support, to which we associate the multivariate local zeta function $Z_{\Phi}(f, s)$. Fix an embedded resolution $\sigma: X \rightarrow \mathbb{K}$ of $D_{\mathbb{K}}=\cup_{i=1}^{m} f_{i}^{-1}(0)$ as in Theorem 2 . Then

(i) $Z_{\Phi}(f, s)$ is convergent and defines a holomorphic function in the region

$$
\sum_{j=1}^{m} N_{f_{j, i}} \operatorname{Re}\left(s_{j}\right)+v_{i}>0, \quad \text { for } i \in T
$$

(ii) $Z_{\Phi}(f, s)$ admits a meromorphic continuation to the whole $\mathbb{C}^{m}$, with poles belonging to

$$
\bigcup_{i \in T} \bigcup_{t \in \mathbb{N}}\left\{\sum_{j=1}^{m} N_{f_{j}, i} s_{j}+v_{i}+t=0\right\}
$$

with $t \in \mathbb{N}$ if $\mathbb{K}=\mathbb{R}$ and $t=\frac{1}{2} \mathbb{N}$ if $\mathbb{K}=\mathbb{C}$, and with poles belonging to

$$
\bigcup_{1 \leq i \leq r}\left\{\sum_{j=1}^{m} a_{j, i} s_{j}+b_{i}=0\right\},
$$

in the p-field case. In addition, in the p-adic case the multivariate local zeta function has a meromorphic continuation as a rational function

$$
\left.Z_{\Phi}(f, s)=\frac{P_{\Phi}(s)}{\prod_{i \in T}\left(1-q^{-\left(\sum_{j=1}^{m} N_{f_{j}, i} s_{j}+v_{i}\right.}\right)}\right)
$$

in $q^{-s_{1}}, \ldots, q^{-s_{m}}$, where $P_{\Phi}(s)$ is a polynomial in the variables $q^{-s_{i}}$.

\section{Meromorphic Continuation of Koba-Nielsen Amplitudes Defined on Local Fields} of Characteristic Zero

The Koba-Nielsen open string amplitudes for $N$-points over a local field $\mathbb{K}$ of characteristic zero are defined as

$$
A_{\mathbb{K}}^{(N)}(\boldsymbol{k}):=\int_{\mathbb{K}^{N-3}} \prod_{i=2}^{N-2}\left|x_{j}\right|_{\mathbb{K}}^{k_{1} k_{j}}\left|1-x_{j}\right|_{\mathbb{K}}^{k_{N-1} k_{j}} \prod_{2 \leq i<j \leq N-2}\left|x_{i}-x_{j}\right|_{\mathbb{K}}^{k_{i} k_{j}} \prod_{i=2}^{N-2} d x_{i},
$$


where $\boldsymbol{k}=\left(\boldsymbol{k}_{1}, \ldots, \boldsymbol{k}_{N}\right), \boldsymbol{k}_{i}=\left(k_{0, i}, \ldots, k_{l, i}\right) \in \mathbb{C}^{l+1}$, for $i=1, \ldots, N$, is the momentum vector of the $i$-th tachyon (with Minkowski product $k_{i} k_{j}=-k_{0, i} k_{0, j}+k_{1, i} k_{1, j}+\cdots+k_{l, i} k_{l, j}$ ), obeying

$$
\sum_{i=1}^{N} \boldsymbol{k}_{i}=\mathbf{0}, \quad \boldsymbol{k}_{i} \boldsymbol{k}_{i}=2 \text { for } i=1, \ldots, N .
$$

The parameter $l$ is typically taken to be 25 . However, we do not require using the critical dimension, thus $l$ can be any positive number. These amplitudes were introduced by Brekke, Freund, Olson and Witten, among others in their works about string amplitudes, see e.g., Section 8 of [4]. In the case $N=4$ and $\mathbb{K}=\mathbb{R}$, the amplitude (87) is the Veneziano amplitude, see [10]. In Section 2 of [48] and [49], it was established that the $N$-point closed string amplitude at the tree level is the product of $A_{\mathbb{C}}^{(N)}(\boldsymbol{k})$ times a polynomial function in the momenta $k$. Hence, the results established in [6] are still valid for closed string amplitudes at tree level.

If we take the Minkowski products of the kinematic parameters as follows $k_{i} \boldsymbol{k}_{j}=s_{i j} \in \mathbb{C}$, the string amplitude (87) becomes to the following integral which is a type of multivariate local zeta function

$$
Z_{\mathbb{K}}^{(N)}(s):=\int_{\mathbb{K}^{N-3} \backslash D_{N}} \prod_{i=2}^{N-2}\left|x_{j}\right|_{\mathbb{K}}^{s_{1 j}}\left|1-x_{j}\right|_{\mathbb{K}}^{s_{(N-1) j}} \prod_{2 \leq i<j \leq N-2}\left|x_{i}-x_{j}\right|_{\mathbb{K}}^{s_{i j}} \prod_{i=2}^{N-2} d x_{i},
$$

where $\prod_{i=2}^{N-2} d x_{i}$ is the normalized Haar measure on $\mathbb{K}^{N-3}, s:=\left(s_{i j}\right) \in \mathbb{C}^{D}$, with $D=\frac{N(N-3)}{2}$ denotes the total number of indices $i j$, and

$$
D_{N}:=\left\{x \in \mathbb{K}^{N-3} ; \prod_{i=2}^{N-2} x_{i} \prod_{i=2}^{N-2}\left(1-x_{i}\right) \prod_{2 \leq i<j \leq N-2}\left(x_{i}-x_{j}\right)=0\right\} .
$$

We have called integrals of type (89) Koba-Nielsen local zeta functions. For simplicity of notation, we put $\mathbb{K}^{N-3}$ instead of $\mathbb{K}^{N-3} \backslash D_{N}$ in (89).

In [6], we establish, in a rigorous mathematical way that the Koba-Nielsen string amplitudes defined on any local field of characteristic zero are bona fide integrals and that they can be extended to meromorphic functions in the kinematic parameters. In order to prove the meromorphic continuation of (89), we express it as linear combinations of local zeta functions. These computations were first made in the case of $\mathbb{K}=\mathbb{R}$, but they can be easily extended to other local fields of characteristic zero. Thus, we only review the real case. We consider $\mathbb{R}^{N-3}$ as an $\mathbb{R}$-analytic manifold, with $N \geq 4$, and use $\left\{x_{2}, \ldots, x_{N-2}\right\}$ as a coordinate system. In order to regularize the integral (89), we use a partition of $\mathbb{R}^{N-3}$ constructed using a smooth function $\chi: \mathbb{R} \rightarrow \mathbb{R}$ satisfying

$$
\chi(x)=\left\{\begin{array}{ccc}
1 & \text { if } & x \in[-2,2] \\
0 & \text { if } & x \in(-\infty,-2-\epsilon] \cup[2+\epsilon,+\infty),
\end{array}\right.
$$

for some fixed positive $\epsilon$ sufficiently small. This function is well-known, see e.g., Section 1.4 of [69], Section 5.2 of [26]. The number 2 was chosen in an arbitrary form, the key point is that the interval $[0,1]$ is included in the locus where $\chi \equiv 1$.

Now, we can write

$$
Z^{(N)}(s)=\sum_{I} Z_{I}^{(N)}(s)
$$

with

$$
Z_{I}^{(N)}(s):=\int_{\mathbb{R}^{N-3}} \varphi_{I}(x) \prod_{j=2}^{N-2}\left|x_{j}\right|^{s_{1 j}} \prod_{j=2}^{N-2}\left|1-x_{j}\right|^{s_{(N-1) j}} \prod_{2 \leq i<j \leq N-2}\left|x_{i}-x_{j}\right|^{s_{i j}} \prod_{i=2}^{N-2} d x_{i},
$$


where the functions $\left\{\varphi_{I}\right\}$ are defined as

$$
\varphi_{I}: \mathbb{R}^{N-3} \rightarrow \mathbb{R} ; x \mapsto \prod_{i \in I} \chi\left(x_{i}\right) \prod_{i \notin I}\left(1-\chi\left(x_{i}\right)\right),
$$

for $I \subseteq\{2, \ldots, N-2\}$, including the empty set, with the convention that $\prod_{i \in \varnothing} \equiv 1$. Notice that $\varphi_{I} \in C^{\infty}\left(\mathbb{R}^{N-3}\right)$ and $\sum_{I} \varphi_{I}(x) \equiv 1$, for $x \in \mathbb{R}^{N-3}$, i.e., the functions $\left\{\varphi_{I}\right\}$ form a partition of the unity.

In the case $I=\{2, \ldots, N-2\}, Z_{I}^{(N)}(s)$ is a classical multivariate Igusa local zeta function (since $\varphi_{I}(x)$ has compact support). It is well known that these integrals are holomorphic functions in a region including $\operatorname{Re}\left(s_{i j}\right)>0$ for all $i j$, and they admit meromorphic continuations to the whole $\mathbb{C}^{D}$, see ([6], Theorem 3.2).

In the case $I \neq\{2, \ldots, N-2\}$, by changing variables in (91) as $x_{i} \rightarrow \frac{1}{x_{i}}$ for $i \notin I$, and $x_{i} \rightarrow x_{i}$ for $i \in I$, we have $\prod_{i=2}^{N-2} d x_{i} \rightarrow \prod_{i \notin I} \frac{1}{\left|x_{i}\right|^{2}} \prod_{i=2}^{N-2} d x_{i}$, and by setting $\widetilde{\chi}\left(x_{i}\right):=$ $1-\chi\left(\frac{1}{x_{i}}\right)$ for $i \notin I$, i.e.,

$$
\widetilde{\chi}\left(x_{i}\right)=\left\{\begin{array}{ccc}
1 & \text { if } & \left|x_{i}\right| \leq \frac{1}{2+\epsilon} \\
0 & \text { if } & \left|x_{i}\right| \geq \frac{1}{2}
\end{array}\right.
$$

we have that supp $\tilde{\chi} \subseteq\left[-\frac{1}{2}, \frac{1}{2}\right]$ and $\tilde{\chi} \in C^{\infty}(\mathbb{R})$. Now setting $\widetilde{\varphi}_{I}(x):=\prod_{i \notin I} \widetilde{\chi}\left(x_{i}\right)$ $\prod_{i \in I} \chi\left(x_{i}\right)$, and

$$
\begin{aligned}
F_{I}(x, s) & :=\prod_{j \in I}\left|x_{j}\right|^{s_{1 j}} \prod_{j=2}^{N-2}\left|1-x_{j}\right|^{s_{(N-1) j}} \prod_{\substack{2 \leq i<j \leq N-2 \\
i, j \in I}}\left|x_{i}-x_{j}\right|^{s_{i j}} \\
\quad \times & \prod_{\substack{2 \leq i<j \leq N-2 \\
i, j \notin I}}\left|x_{i}-x_{j}\right|^{s_{i j}} \prod_{\substack{2 \leq i<j \leq N-2 \\
i \notin I, j \in I}}\left|1-x_{i} x_{j}\right|^{s_{i j}} \prod_{\substack{2 \leq i<j \leq N-2 \\
i \in I, j \notin I}}\left|1-x_{i} x_{j}\right|^{s_{i j}},
\end{aligned}
$$

we have

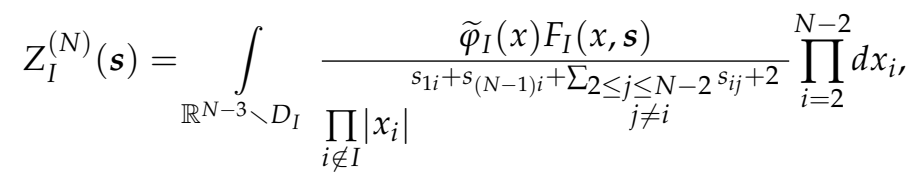

where $D_{I}$ is the divisor defined by the polynomial

$$
\begin{aligned}
\prod_{i=2}^{N-2} x_{i} \prod_{i=2}^{N-2}\left(1-x_{i}\right) \prod_{\substack{2 \leq i<j \leq N-2 \\
i, j \in I}}\left(x_{i}-x_{j}\right) & \prod_{\substack{2 \leq i<j \leq N-2 \\
i, j \notin I}}\left(x_{i}-x_{j}\right) \\
& \times \prod_{\substack{2 \leq i<j \leq N-2 \\
i \notin I, j \in I}}\left(1-x_{i} x_{j}\right) \prod_{\substack{2 \leq i<j \leq N-2 \\
i \in I, j \notin I}}\left(1-x_{i} x_{j}\right) .
\end{aligned}
$$

The integrals $Z_{I}^{(N)}(s)$, with $I \neq\{2, \ldots, N-2\}$, are not classical multivariate local zeta functions thus we do not apply the theory of local zeta functions. Thus, in [6] we show that they define holomorphic functions on some nonempty open in $\mathbb{C}^{D}$, and admit meromorphic continuations to the whole $\mathbb{C}^{D}$.

Lemma 1. (Lemma 4.1 of [6]) For any $I \subseteq\{2, \ldots, N-2\}$, the function $Z_{I}^{(N)}(s)$ is holomorphic in $s$ on the solution set $\mathcal{H}(I)$ of a system of inequalities of the form

$$
\mathcal{H}(I):=\left\{s_{i j} \in \mathbb{C}^{D} ; \sum_{i j \in M(I)} N_{i j, k}(I) \operatorname{Re}\left(s_{i j}\right)+\gamma_{k}(I)>0, \text { for } k \in T(I)\right\} \neq \varnothing,
$$


where $N_{i j, k}(I), \gamma_{k}(I) \in \mathbb{Z}$, and $M(I), T(I)$ are finite sets. More precisely, for each $k$, either all numbers $N_{i j, k}(I)$ are equal to 0 or 1 and $\gamma_{k}(I)>0$, or all numbers $N_{i j, k}(I)$ are equal to 0 or -1 and $\gamma_{k}(I)<0$.

In addition, $Z_{I}^{(N)}(s)$ admits an analytic continuation to the whole $\mathbb{C}^{D}$, as a meromorphic function with poles belonging to

$$
\mathcal{P}(I):=\bigcup_{t \in \mathbb{N}} \bigcup_{k \in T(I)}\left\{s_{i j} \in \mathbb{C}^{D} ; \sum_{i j \in M(I)} N_{i j, k}(I) s_{i j}+\gamma_{k}(I)+t=0\right\} .
$$

To show the existence of the meromorphic continuation of $Z^{(N)}(s)$ it is necessary that all the integrals $Z_{I}^{(N)}(s)$ be holomorphic in a common domain, and then Formula (90) allows us to construct a meromorphic continuation of $Z^{(N)}(s)$. We show that $\cap_{I} \mathcal{H}(I)$ contains a nonempty open subset of $\mathbb{C}^{D}$ by studying the possible poles of integrals

$$
Z_{I}^{(N)}(s):=\left.Z_{I}^{(N)}(s)\right|_{s_{i j}=s}
$$

for any $I$, and proving that $Z^{(N)}(s):=\left.Z^{(N)}(s)\right|_{s_{i j}=s}$ is a holomorphic function in the region

$$
-\frac{2}{N-2}<\operatorname{Re}(s)<-\frac{2}{N}
$$

This fact was proved in Theorem 4.1 in [6].

Furthermore, the Koba-Nielsen local zeta function $Z^{(N)}(s)$ is convergent and holomorphic in the region determined by the following inequalities:

$$
\operatorname{Re}\left(s_{i j}\right)>-1
$$

for all $\frac{N(N-3)}{2}$ variables $s_{i j}$,

$$
\sum_{j \in J} \operatorname{Re}\left(s_{1 j}\right)+\sum_{\substack{i, j \in J \\ i<j}} \operatorname{Re}\left(s_{i j}\right)>-\# J
$$

for all subsets $J \subset\{2, \ldots, N-2\}$ with $\# J \geq 2$,

$$
\sum_{j \in J} \operatorname{Re}\left(s_{(N-1) j}\right)+\sum_{\substack{i, j \in J \\ i<j}} \operatorname{Re}\left(s_{i j}\right)>-\# J
$$

for all subsets $J \subset\{2, \ldots, N-2\}$ with $\# J \geq 2$,

$$
\sum_{\substack{i, j \in J \\ i<j}} \operatorname{Re}\left(s_{i j}\right)>-\# J+1
$$

for all subsets $J \subset\{2, \ldots, N-2\}$ with $\# J \geq 3$,

$$
\sum_{j \in J} \operatorname{Re}\left(s_{1 j}\right)+\sum_{j \in J} \operatorname{Re}\left(s_{(N-1) j}\right)+\sum_{\substack{i \in\{2, \ldots, N-2\} \backslash J \\ j \in J}} \operatorname{Re}\left(s_{i j}\right)+\sum_{\substack{i, j \in J \\ i<j}} \operatorname{Re}\left(s_{i j}\right)<-\# J
$$

for all subsets $J \subset\{2, \ldots, N-2\}$ with $\sharp J \geq 1$. This region contains the open subset defined by

$$
-\frac{2}{N-2}<\operatorname{Re}\left(s_{i j}\right)<-\frac{2}{N^{\prime}}
$$

for all indices $i j$.

As in the $p$-adic case, we use the meromorphic continuation of (89) to the whole $\mathbb{C}^{D}$, which is denoted by $Z_{\mathbb{K}}^{(N)}(s)$, as regularizations of the amplitudes $A_{\mathbb{K}}^{(N)}(\boldsymbol{k})$ by redefining 


$$
A_{\mathbb{K}}^{(N)}(\boldsymbol{k})=\left.Z_{\mathbb{K}}^{(N)}(\boldsymbol{s})\right|_{s_{i j}=k_{i} \boldsymbol{k}_{j}}
$$

see Theorem 6.1 of [6]. It is important to mention here that in the regularization of $A_{\mathbb{K}}^{(N)}(\boldsymbol{k})$, we do not use the kinematic restrictions (88).

Furthermore, in [6] we show that $A_{\mathbb{K}}^{(N)}(\boldsymbol{k})$ converges on some open subset of $\mathbb{C}^{(N-1)(l+1)}$ by showing that this open is mapped into the domain of convergency of $Z_{\mathbb{K}}^{(N)}(s)$ by $\boldsymbol{k} \rightarrow s_{i j}=\boldsymbol{k}_{i} \boldsymbol{k}_{j}$. In addition, we prove that $A_{\mathbb{K}}^{(N)}(\boldsymbol{k})$ extends to a meromorphic function to the whole $\mathbb{C}^{(N-1)(l+1)}$, and that its polar set is contained in the inverse image of the polar set of $Z_{\mathbb{K}}^{(N)}(s)$ under that mapping, where the possible poles are described in terms of numerical data of suitable resolutions of singularities:

Theorem 4. (Theorem 7.1 of [6]) Let $\mathbb{K}$ be a local field of characteristic zero. The integral $A_{\mathbb{K}}^{(N)}(\boldsymbol{k})$ converges and is holomorphic in the open set $\mathcal{U} \subset \mathbb{C}^{(N-1)(l+1)}$. It extends to a meromorphic function in $\boldsymbol{k}$ on the whole $\mathbb{C}^{(N-1)(l+1)}$.

If $\mathbb{K}$ is an $\mathbb{R}$-field, then the possible poles of $A_{\mathbb{K}}^{(N)}(\boldsymbol{k})$ belong to

$$
\bigcup_{I \subseteq\{2, \ldots, N-2\}} \bigcup_{t \in \mathbb{N}} \bigcup_{r \in T(I)}\left\{k \in \mathbb{C}^{(N-1)(l+1)} ; \sum_{i j \in M(I)} N_{i j, r}(I) \boldsymbol{k}_{i} \boldsymbol{k}_{j}+\gamma_{r}(I)+\frac{t}{[\mathbb{K}: \mathbb{R}]}=0\right\},
$$

where $N_{i j, r}(I), \gamma_{r}(I) \in \mathbb{Z}$, and $M(I), T(I)$ are finite sets, and $[\mathbb{K}: \mathbb{R}]=1$ if $\mathbb{K}=\mathbb{R}$, and $[\mathbb{K}: \mathbb{R}]=$ 2 if $\mathbb{K}=\mathbb{C}$. If $\mathbb{K}$ is a $p$-adic field, then $A_{\mathbb{K}}^{(N)}(\boldsymbol{k})$ is a rational function in the variables $q^{-\boldsymbol{k}_{i} \boldsymbol{k}_{j}}$, and its possible poles belong to

$$
\bigcup_{I \subseteq\{2, \ldots, N-2\}} \bigcup_{r \in T(I)}\left\{k \in \mathbb{C}^{(N-1)(l+1)} ; \sum_{i j \in M(I)} N_{i j, r}(I) \operatorname{Re}\left(\boldsymbol{k}_{i} \boldsymbol{k}_{j}\right)+\gamma_{r}(I)=0\right\} .
$$

More precisely, for each $r$, either all numbers $N_{i j, r}(I)$ are equal to 0 or 1 and $\gamma_{r}(I)>0$, or all numbers $N_{i j, r}(I)$ are equal to 0 or -1 and $\gamma_{r}(I)<0$.

We now show explicitly the existence of a meromorphic continuation for $Z_{\mathbb{R}}^{(N)}(s)$ in the cases $N=4,5$, by using Hironaka's resolution of singularities theorem.

\subsection{Example: 4-Point Koba-Nielsen String Amplitude}

The 4-point Koba-Nielsen open string amplitude is defined as

$$
Z^{(4)}(s)=\int_{\mathbb{R}}\left|x_{2}\right|^{s_{12}}\left|1-x_{2}\right|^{s_{32}} d x_{2} .
$$

Using the function

$$
\chi\left(x_{2}\right)=\left\{\begin{array}{ccc}
1 & \text { if } & x_{2} \in[-2,2] \\
0 & \text { if } & x_{2} \in(-\infty,-2-\epsilon] \cup[2+\epsilon,+\infty),
\end{array}\right.
$$

where $\epsilon>0$ is sufficiently small, we construct the following partition of the unity:

$$
\begin{array}{cl}
\varphi_{\{2\}}: & \mathbb{R} \rightarrow \mathbb{R} ; x_{2} \mapsto \chi\left(x_{2}\right) \\
\varphi_{\varnothing}: & \mathbb{R} \rightarrow \mathbb{R} ; \quad x_{2} \mapsto 1-\chi\left(x_{2}\right)
\end{array}
$$

Notice that $\varphi_{\varnothing}, \varphi_{\{2\}} \in C^{\infty}(\mathbb{R})$ and $\varphi_{\{2\}}(x)+\varphi_{\varnothing}(x) \equiv 1$, for $x \in \mathbb{R}$. Hence, 


$$
Z^{(4)}(s)=Z_{\{2\}}^{(4)}(s)+Z_{\varnothing}^{(4)}(s),
$$

with

$$
Z_{\{2\}}^{(4)}(s)=\int_{\mathbb{R}} \chi\left(x_{2}\right)\left|x_{2}\right|^{s_{12}}\left|1-x_{2}\right|^{s_{32}} d x_{2}
$$

and

$$
Z_{\varnothing}^{(4)}(s)=\int_{\mathbb{R}}\left(1-\chi\left(x_{2}\right)\right)\left|x_{2}\right|^{s_{12}}\left|1-x_{2}\right|^{s_{32}} d x_{2} .
$$

The integral (98) is a multivariate local zeta function since $\chi\left(x_{2}\right)$ has compact support. By a classical result of local zeta functions $Z_{\{2\}}^{(4)}(s)$ converges when $\operatorname{Re}\left(s_{12}\right)>-1$ and $\operatorname{Re}\left(s_{32}\right)>-1$. Furthermore, it admits a meromorphic continuation to the whole $\mathbb{C}^{2}$, see Theorem 3.2 of [6].

The second integral (99) is not a multivariate local zeta function but it can be transformed in one by changing of variables as $x_{2} \rightarrow \frac{1}{x_{2}}$, then $d x_{2} \rightarrow \frac{1}{\left|x_{2}\right|^{2}} d x_{2}$, and by setting $\tilde{\chi}\left(x_{2}\right):=1-\chi\left(\frac{1}{x_{2}}\right)$, we have that

$$
\tilde{\chi}\left(x_{2}\right)=\left\{\begin{array}{ccc}
1 & \text { if } & \left|x_{2}\right| \leq \frac{1}{2+\epsilon} \\
0 & \text { if } & \left|x_{2}\right| \geq \frac{1}{2}
\end{array} .\right.
$$

Notice that the support of $\widetilde{\chi}$ is contained in $\left[-\frac{1}{2}, \frac{1}{2}\right]$ and $\widetilde{\chi} \in C^{\infty}(\mathbb{R})$. Thus, integral (99) becomes

$$
Z_{\varnothing}^{(4)}(s)=\int_{\mathbb{R}} \tilde{\chi}\left(x_{2}\right)\left|x_{2}\right|^{-s_{12}-s_{32}-2}\left|1-x_{2}\right|^{s_{32}} d x_{2},
$$

which is analytic when $-\operatorname{Re}\left(s_{12}\right)-\operatorname{Re}\left(s_{32}\right)-1>0$ and $\operatorname{Re}\left(s_{32}\right)+1>0$. We concluded that $Z^{(4)}(s)$ is analytic in the region

$$
\operatorname{Re}\left(s_{12}\right)>-1, \quad \operatorname{Re}\left(s_{32}\right)>-1, \quad \operatorname{Re}\left(s_{12}\right)+\operatorname{Re}\left(s_{32}\right)<-1 .
$$

Which contains the open set $-1<\operatorname{Re}\left(s_{12}\right)<-\frac{1}{2}$ and $-1<\operatorname{Re}\left(s_{32}\right)<-\frac{1}{2}$.

\subsection{Example: 5-Point Koba-Nielsen String Amplitude}

Fix $N=5$. By using the function $\chi(x)$, see (97), we define the following partition of the unity:

$$
\begin{aligned}
\varphi_{\{2,3\}}: & \mathbb{R}^{2} \rightarrow \mathbb{R} ; \quad\left(x_{2}, x_{3}\right) \mapsto \chi\left(x_{2}\right) \chi\left(x_{3}\right), \\
\varphi_{\{2\}}: & \mathbb{R}^{2} \rightarrow \mathbb{R} ; \quad\left(x_{2}, x_{3}\right) \mapsto \chi\left(x_{2}\right)\left(1-\chi\left(x_{3}\right)\right), \\
\varphi_{\{3\}}: & \mathbb{R}^{2} \rightarrow \mathbb{R} ; \quad\left(x_{2}, x_{3}\right) \mapsto \chi\left(x_{3}\right)\left(1-\chi\left(x_{2}\right)\right), \\
\varphi_{\varnothing}: & \mathbb{R}^{2} \rightarrow \mathbb{R} ; \quad\left(x_{2}, x_{3}\right) \mapsto\left(1-\chi\left(x_{2}\right)\right)\left(1-\chi\left(x_{3}\right)\right) .
\end{aligned}
$$

Then $Z^{(5)}(s)=Z_{\{2,3\}}^{(5)}(s)+Z_{\{3\}}^{(5)}(s)+Z_{\{2\}}^{(5)}(s)+Z_{\varnothing}^{(5)}(s)$. We consider the first integral

$$
Z_{\{2,3\}}^{(5)}(s)=\int_{\mathbb{R}^{2}} \chi\left(x_{2}\right) \chi\left(x_{3}\right)\left|x_{2}\right|^{s_{12}}\left|x_{3}\right|^{s_{13}}\left|1-x_{2}\right|^{s_{42}}\left|1-x_{3}\right|^{s_{43}}\left|x_{2}-x_{3}\right|^{s_{23}} d x_{2} d x_{3} .
$$

Since $\varphi_{\{2,3\}}$ has compact support, then integral (100) is a local zeta function. Thus, we use resolution of singularities of the divisor $D_{5}$ defined by $x_{2} x_{3}\left(1-x_{2}\right)\left(1-x_{3}\right)\left(x_{2}-x_{3}\right)=0$. This arrangement is not locally monomial only at the points $(0,0)$ and $(1,1)$. Hence, we pick a partition of the unity, $\sum_{i=0}^{2} \Omega_{i}\left(x_{2}, x_{3}\right)=1$, and we write

$$
Z_{\{2,3\}}^{(5)}(s)=\sum_{j=0}^{2} Z_{\Omega_{j}}^{(5)}(s),
$$


where

$$
Z_{\Omega_{j}}^{(5)}(s)=\int_{\mathbb{R}^{2}} \Omega_{j}\left(x_{2}, x_{3}\right)\left|x_{2}\right|^{s_{12}}\left|x_{3}\right|^{s_{13}}\left|1-x_{2}\right|^{s_{42}}\left|1-x_{3}\right|^{s_{43}}\left|x_{2}-x_{3}\right|^{s_{23}} d x_{2} d x_{3} .
$$

We assume that $\Omega_{0}$ and $\Omega_{1}$ are smooth functions with support in a small neighborhood of $(0,0)$ and $(1,1)$, respectively. So, we only compute the integrals $Z_{\Omega_{0}}^{(5)}(s)$ and $Z_{\Omega_{1}}^{(5)}(s)$.

In terms of convergence and holomorphy, around $(0,0)$, the factor $\left|1-x_{2}\right|^{s_{42}}\left|1-x_{3}\right|^{s_{43}}$ can be neglected, hence we only need an embedded resolution of $x_{2} x_{3}\left(x_{2}-x_{3}\right)=0$, which is obtained by a blow-up at the origin. It means to make the changes of variables

$$
\begin{aligned}
\sigma_{0}: \mathbb{R}^{2} \rightarrow \mathbb{R}^{2} ; u_{2} & \mapsto x_{2}=u_{2} \\
u_{3} & \mapsto x_{3}=u_{2} u_{3} .
\end{aligned}
$$

Hence integral $Z_{\Omega_{0}}^{(5)}(s)$ becomes

$$
\int_{\mathbb{R}^{2}}\left(\Omega_{0} \circ \sigma_{0}\right)\left(u_{2}, u_{3}\right)\left|u_{2}\right|^{s_{12}+s_{13}+s_{23}+1}\left|u_{3}\right|^{s_{13}}\left|1-u_{3}\right|^{s_{23}} g(u, s) d u_{2} d u_{3},
$$

where $g(u, s)$ is invertible on the support of $\Omega_{0} \circ \sigma_{0}$ and can thus be neglected from the point of view of convergence and holomorphy. Hence, by ([6], Lemma 3.1), we obtain the convergence conditions:

$$
\operatorname{Re}\left(s_{12}\right)+\operatorname{Re}\left(s_{13}\right)+\operatorname{Re}\left(s_{23}\right)+2>0, \quad \operatorname{Re}\left(s_{23}\right)+1>0, \quad \operatorname{Re}\left(s_{13}\right)+1>0 .
$$

We can take other chart of the blow-up, i.e., the change of variables

$$
\begin{aligned}
\sigma_{0}^{\prime}: \mathbb{R}^{2} \rightarrow \mathbb{R}^{2} ; u_{2} & \mapsto x_{2}=u_{2} u_{3} \\
u_{3} & \mapsto x_{3}=u_{3},
\end{aligned}
$$

with this change yields the same first and second condition and

$$
\operatorname{Re}\left(s_{12}\right)+1>0 .
$$

By symmetry we can consider any of these changes of variables. Completely similarly, for the convergence of $Z_{\Omega_{1}}^{(5)}(s)$, we need also the new conditions

$$
\operatorname{Re}\left(s_{42}\right)+\operatorname{Re}\left(s_{43}\right)+\operatorname{Re}\left(s_{23}\right)+2>0, \quad \operatorname{Re}\left(s_{42}\right)+1>0, \quad \operatorname{Re}\left(s_{43}\right)+1>0 .
$$

The conditions coming from the locally monomial integral $Z_{\Omega_{2}}^{(5)}(s)$ are already included.

The integral $Z_{\{3\}}^{(5)}(s)$ is not a multivariate local zeta function, so we take the following change of variables $x_{2} \rightarrow \frac{1}{x_{2}}$, then we have $d x_{2} \rightarrow \frac{1}{\left|x_{2}\right|^{2}} d x_{2}$, and by setting $\widetilde{\chi}\left(x_{2}\right):=1-\chi\left(\frac{1}{x_{2}}\right)$, we have that

$$
\widetilde{\chi}\left(x_{2}\right)=\left\{\begin{array}{ccc}
1 & \text { if } & \left|x_{2}\right| \leq \frac{1}{2+\epsilon} \\
0 & \text { if } & \left|x_{2}\right| \geq \frac{1}{2}
\end{array} .\right.
$$

Then 


$$
\begin{aligned}
Z_{\{3\}}^{(5)}(s)=\int_{\mathbb{R}^{2}} \tilde{\chi}\left(x_{2}\right) \chi\left(x_{3}\right)\left|x_{2}\right|^{-s_{12}-s_{42}-s_{23}-2}\left|x_{3}\right|^{s_{13}}\left|1-x_{2}\right|^{s_{42}} \mid 1- & \left.x_{3}\right|^{s_{43}} \\
& \times\left|1-x_{2} x_{3}\right|^{s_{23}} d x_{2} d x_{3} .
\end{aligned}
$$

Since $x_{2} x_{3}\left(1-x_{2}\right)\left(1-x_{3}\right)\left(1-x_{2} x_{3}\right)$ is locally monomial in the support of $\tilde{\chi}\left(x_{2}\right) \chi\left(x_{3}\right)$, the only new condition is

$$
-\operatorname{Re}\left(s_{12}\right)-\operatorname{Re}\left(s_{42}\right)-\operatorname{Re}\left(s_{23}\right)-1>0
$$

Completely analogously, $Z_{\{2\}}^{(5)}(s)$ induces the extra condition

$$
-\operatorname{Re}\left(s_{13}\right)-\operatorname{Re}\left(s_{43}\right)-\operatorname{Re}\left(s_{23}\right)-1>0
$$

For the last integral, we are setting $\widetilde{\chi}\left(x_{2}\right):=1-\chi\left(\frac{1}{x_{2}}\right)$ and $\widetilde{\chi}\left(x_{3}\right):=1-\chi\left(\frac{1}{x_{3}}\right)$. Thus,

$$
\begin{aligned}
& Z_{\varnothing}^{(5)}(s)=\int_{\mathbb{R}^{2}} \tilde{\chi}\left(x_{2}\right) \widetilde{\chi}\left(x_{3}\right)\left|x_{2}\right|^{-s_{12}-s_{42}-s_{23}-2}\left|x_{3}\right|^{-s_{13}-s_{43}-s_{23}-2}\left|1-x_{2}\right|^{s_{42}}\left|1-x_{3}\right|^{s_{43}} \\
& \times\left|x_{2}-x_{3}\right|^{s_{23}} d x_{2} d x_{3} \text {. }
\end{aligned}
$$

In this case, we have the same divisor as for $Z_{\{2,3\}}^{(5)}(s)$; the differences are the powers of $\left|x_{2}\right|$ and $\left|x_{3}\right|$ and the function $\tilde{\chi}\left(x_{2}\right) \tilde{\chi}\left(x_{3}\right)$ that does not contain $(1,1)$ in its support. Hence, the only new condition will arise from the blow-up at the origin, namely

$$
-\operatorname{Re}\left(s_{12}\right)-\operatorname{Re}\left(s_{42}\right)-\operatorname{Re}\left(s_{23}\right)-\operatorname{Re}\left(s_{13}\right)-\operatorname{Re}\left(s_{43}\right)-2>0 .
$$

Hence $Z^{(5)}(s)$ is analytic in the region defined by conditions (101)-(106), i.e.,

$$
\begin{array}{ll}
\operatorname{Re}\left(s_{i j}\right)>-1, \text { for all ij } & \\
\operatorname{Re}\left(s_{12}\right)+\operatorname{Re}\left(s_{13}\right)+\operatorname{Re}\left(s_{23}\right)>-2, & \operatorname{Re}\left(s_{42}\right)+\operatorname{Re}\left(s_{43}\right)+\operatorname{Re}\left(s_{23}\right)>-2, \\
\operatorname{Re}\left(s_{12}\right)+\operatorname{Re}\left(s_{42}\right)+\operatorname{Re}\left(s_{23}\right)<-1, & \operatorname{Re}\left(s_{13}\right)+\operatorname{Re}\left(s_{43}\right)+\operatorname{Re}\left(s_{23}\right)<-1, \\
\sum_{i j} \operatorname{Re}\left(s_{i j}\right)<-2 . &
\end{array}
$$

This region of convergence contains the open subset defined by

$$
-\frac{2}{3}<\operatorname{Re}\left(s_{i j}\right)<-\frac{2}{5} \quad \text { for all } i j
$$

Then, in particular, $Z^{(5)}(s)$ is analytic in the interval $-\frac{2}{3}<\operatorname{Re}(s)<-\frac{2}{5}$.

Author Contributions: Writing-review and editing: M.B.-G., H.G.-C., E.Y.L., W.A.Z.-G. All the authors contributed to the manuscript equally. All authors have read and agreed to the published version of the manuscript.

Funding: The first author was partially supported by the University of Guadalajara. The third author was supported by a Conacyt fellowship. The fourth author was partially supported by Conacyt Grant No. 250845 (Mexico) and by the Debnath Endowed Professorship (UTRGV, USA).

Data Availability Statement: No new data were created or analyzed in this study. Data sharing is not applicable to this article.

Conflicts of Interest: The authors declare no conflict of interest. 


\section{References}

1. Schlotterer, O. The Number Theory of Superstring Amplitudes. In Periods in Quantum Field Theory and Arithmetic. ICMAT-MZV 2014; Burgos, G.J., Ebrahimi-Fard, K., Gangl, H., Eds.; Springer Proceedings in Mathematics \& Statistics; Springer: Cham, Switzerland, 2020; Volume 314, pp. 77-103.

2. Stieberger, S. Periods and Superstring Amplitudes. In Periods in Quantum Field Theory and Arithmetic. ICMAT-MZV 2014; Burgos, G.J., Ebrahimi-Fard, K., Gangl, H., Eds.; Springer Proceedings in Mathematics \& Statistics; Springer: Cham, Switzerland, 2020; Volume 314, pp. 45-76.

3. Vladimirov, V.S.; Volovich, I.V.; Zelenov, E.I. p-Adic Analysis and Mathematical Physics; World Scientific: Singapore, 1994.

4. Brekke, L.; Freund, P.G.O. p-adic numbers in physics. Phys. Rep. 1993, 233, 1-66. [CrossRef]

5. Hloušek, Z.; Spector, D. p-Adic string theories. Ann. Phys. 1989, 189, 370-431. [CrossRef]

6. Bocardo-Gaspar, M.; Veys, W.; Zuñiga-Galindo, W.A. Meromorphic continuation of Koba-Nielsen string amplitudes. J. High Energy Phys. 2020, 2020, 1-44.. [CrossRef]

7. García-Compeán, H.; López, E.Y.; Zúñiga-Galindo, W.A. p-Adic open string amplitudes with Chan-Paton factors coupled to a constant B-field. Nucl. Phys. B 2020, 951, 114904.

8. Bocardo-Gaspar, M.; Garcia-Compean, H.; Zúñ iga-Galindo, W.A. Regularization of $p$-adic String Amplitudes, and Multivariate Local Zeta Functions. Lett. Math. Phys. 2019, 109, 1167-1204. [CrossRef]

9. Bocardo-Gaspar, M.; Garcia-Compean, H.; Zúñiga-Galindo, W.A. p-Adic string amplitudes in the limit $p$ approaches to one. J. High Energy Phys. 2018, 2018, 1-23. [CrossRef]

10. Veneziano, G. Construction of a crossing-symmetric, Reggeon-behaved amplitude for linearly rising trajectories. Il Nuovo Cimento A 1968, 57, 190-197. [CrossRef]

11. Virasoro, M. Alternative constructions of crossing-symmetric amplitudes with Regge behavior. Phys. Rev. 1969, 177, $2309-2311$. [CrossRef]

12. Koba, Z.; Nielsen, H. Reaction amplitude for N-mesons: A generalization of the Veneziano-Bardakçi-Ruegg-Virasoro model. Nucl Phys. B 1969, 10, 633-655. [CrossRef]

13. Volovich, I.V. p-Adic string. Class. Quantum Gravity 1987, 4, L83-L87. [CrossRef]

14. Brekke, L.; Freund, P.G.O.; Olson, M.; Witten, E. Non-archimedean String Dynamics. Nucl. Phys. B 1988, 302, 365-402. [CrossRef]

15. Dragovich, B.; Khrennikov, A.Y.; Kozyrev, S.V.; Volovich, I.V.; Zelenov, E.I. p-Adic Mathematical Physics: The First 30 Years. P-Adic Num. Ultrametr. Anal. Appl. 2017, 9, 87-121. [CrossRef]

16. Gubser, S.S.; Knaute, J.; Parikh, S.; Samberg, A.; Witaszczyk, P. p-Adic AdS/CFT. Commun. Math. Phys. 2017, 352, 1019. [CrossRef]

17. Heydeman, M.; Marcolli, M.; Saberi, I.; Stoica, B. Tensor networks, $p$-adic fields, and algebraic curves: Arithmetic and the $\mathrm{AdS}_{3} / \mathrm{CFT}_{2}$ correspondence. Adv. Theor. Math. Phys. 2018, 22, 93-176. [CrossRef]

18. Gubser, S.S.; Heydeman, M.; Jepsen, C.; Marcolli, M.; Parikh, S.; Saberi, I.; Stoica, B.; Trundy, B. Edge length dynamics on graphs with applications to $p$-adic AdS/CFT. J. High Energy Phys. 2017, 2017, 1-35. [CrossRef]

19. Dutta, P.; Ghoshal, D.; Lala, A. Notes on the Exchange Interactions in Holographic p-adic CFT. Phys. Lett. B 2017, 773, 283-289. [CrossRef]

20. Freund, P.G.O.; Olson, M. Non-archimedean Strings. Phys. Lett. B 1987, 199, 186-190. [CrossRef]

21. Freund, P.G.O.; Witten, E. Adelic String Amplitudes. Phys. Lett. B 1987, 199, 191-194. [CrossRef]

22. Frampton, P.H.; Okada, Y. The p-adic String N-Point Function. Phys. Rev. Lett. 1988, 60, 484-486. [CrossRef]

23. Spokoiny, B.L. Quantum Geometry of Nonarchimedean Particles and Strings. Phys. Lett. B 1988, 208, 401-405. [CrossRef]

24. Zabrodin, A.V. Nonarchimedean Strings and Bruhat-tits Trees. Commun. Math. Phys. 1989, 123, 463-483. [CrossRef]

25. Igusa, J.-I. Forms of Higher Degree; Tata Institute of Fundamental Research Lectures on Mathematics and Physics, 59; Tata Institute of Fundamental Research: Bombay, India; Narosa Publishing House: New Delhi, India, 1978.

26. Igusa, J.-I. An Introduction to the Theory of Local Zeta Functions; AMS/IP Studies in Advanced Mathematics, 14; American Mathematical Society: Providence, RI, USA; International Press: Cambridge, MA, USA, 2000.

27. Meuser, D. A survey of Igusa's local zeta function. Am. J. Math. 2016, 138, 149-179. [CrossRef]

28. Denef, J. Report on Igusa's Local Zeta Function. Séminaire Bourbaki, Vol. 1990/91, Exp. No.730-744. Astérisque 1991, $201-203,359-386$.

29. Gerasimov, A.A.; Shatashvili, S.L. On exact tachyon potential in open string field theory. J. High Energy Phys. 2000, 34. [CrossRef]

30. Witten, E. On background independent open string field theory. Phys. Rev. D 1992, 46, 5467-5473. [CrossRef]

31. Witten, E. Some computations in background independent off-shell string theory. Phys. Rev. D 1993, 47, 3405-3410. [CrossRef] [PubMed]

32. Minahan, J.A.; Zwiebach, B. Field theory models for tachyon and gauge field string dynamics. J. High Energy Phys. 2000, 29. [CrossRef]

33. Ghoshal, D. Exact noncommutative solitons in p-adic strings and BSFT. J. High Energy Phys. 2004, 41. [CrossRef]

34. Ghoshal, D. p-Adic string theories provide lattice discretization to the ordinary string worldsheet. Phys. Rev. Lett. 2006, 97, 151601. [CrossRef]

35. Denef, J.; Loeser, F. Caractéristiques D’Euler-Poincaré, Fonctions Zeta locales et modifications analytiques. J. Am. Math. Soc. 1992, 5, 705-720.

36. Loeser, F. Fonctions zêta locales d'Igusa à plusieurs variables, intégration dans les fibres, et discriminants. Ann. Sci. École Norm. Sup. 1989, 22, 435-471. [CrossRef] 
37. Gel'fand, I.M.; Shilov, G.E. Generalized Functions; Academic Press: New York, NY, USA; London, UK, 1977 ; Volume 1.

38. Kashiwara, M.; Kawai, T. On holonomic systems for $\Pi_{l=1}^{N}\left(f_{l}+(\sqrt{ }-1) 0\right)^{\lambda_{l}}$. Publ. Res. Inst. Math. Sci. 1979, 15, 551-575. [CrossRef]

39. Veys, W.; Zúñiga-Galindo, W.A. Zeta functions and oscillatory integrals for meromorphic functions. Adv. Math. 2017, 311, 295-337. [CrossRef]

40. Veys, W.; Zúñiga-Galindo, W.A. Zeta functions for analytic mappings, log-principalization of ideals, and Newton polyhedra. Trans. Am. Math. Soc. 2008, 360, 2205-2227. [CrossRef]

41. Denef, J.; Loeser, F. Motivic Igusa zeta functions. J. Algebr. Geom. 1998, 7, 505-537.

42. Denef, J.; Loeser, F. Germs of arcs on singular algebraic varieties and motivic integration. Invent. Math. 1999, 135, $201-232$. [CrossRef]

43. Atiyah, M.F. Resolution of Singularities and Division of Distributions. Commun. Pure Appl. Math. 1970, 23, 145-150. [CrossRef]

44. Bernstein, I.N. Modules over the ring of differential operators; the study of fundamental solutions of equations with constant coefficients. Funct. Anal. Appl. 1972, 5, 1-16.

45. Zúñiga-Galindo, W.A. Pseudodifferential Equations over Non-Archimedean Spaces; Lectures Notes in Mathematics; Springer: Cham, Switzerland, 2016; Volume 2174.

46. Khrennikov, A.; Kozyrev, S.; Zúñ iga-Galindo, W.A. Ultrametric Pseudodifferential Equations and Applications; Encyclopedia of Mathematics and its Applications, 168; Cambridge University Press: Cambridge, UK, 2018.

47. Mendoza-Martínez, M.L.; Vallejo, J.A.; Zúñiga-Galindo, W.A. Acausal quantum theory for non-Archimedean scalar fields. Rev. Math. Phys. 2019, 31, 1950011. [CrossRef]

48. Kawai, H.; Lewellen, D.C.; Tye, S.H.H. A relation between tree amplitudes of closed and open strings. Nucl. Phys. B 1986, 269, 1-23. [CrossRef]

49. Blumenhagen, R.; Lüst, D.; Theisen, S. String Scattering Amplitudes and Low Energy Effective Field Theory, chapter 16. In Basic in Concepts of String Theory Part of the Series Theoretical and Mathematical Physics; Springer: Berlin/Heidelberg, Germany, 2012 ; pp. 585-639.

50. Hironaka, H. Resolution of singularities of an algebraic variety over a field of characteristic zero. Ann. Math. 1964, 79, 109-326. [CrossRef]

51. Aref'eva, I.Y.; Dragovic, B.G.; Volovich, I.V. On the adelic string amplitudes. Phys. Lett. B 1988, 209, 445-450. [CrossRef]

52. Gusein-Zade, S.M. Integration with respect to the Euler characteristic and its applications. Russ. Math. Surv. 2010, 65, 399-432. [CrossRef]

53. Green, M.B.; Schwarz, J.H.; Witten, E. Superstring Theory; Cambridge University Press: Cambridge, UK, 2012 ; Volume 1.

54. Tong, D. Lectures on String Theory. arXiv 2009, arXiv:0908.0333.

55. Polchinski, J. String Theory. Vol. 1: An Introduction to the Bosonic String; Cambridge University Press: Cambridge, UK, 1998.

56. Fradkin, E.S.; Tseytlin, A.A. Nonlinear Electrodynamics from Quantized Strings. Phys. Lett. B 1985, 163, 123-130. [CrossRef]

57. Abouelsaood, A.; Callan, C.G., Jr.; Nappi, C.R.; Yost, S.A. Open Strings in Background Gauge Fields. Nucl. Phys. B 1987, 280 , 599-624. [CrossRef]

58. Seiberg, N.; Witten, E. String theory and noncommutative geometry. J. High Energy Phys. 1999, 32. [CrossRef]

59. Fairlie, D.B.; Jones, K. Integral representations for the complete four- and five-point veneziano amplitudes. Nucl. Phys. B 1970, 15, 323-330. [CrossRef]

60. Ghoshal, D.; Kawano, T. Towards p-adic string in constant B-field. Nucl. Phys. B 2005, 710, 577-598. [CrossRef]

61. Albeverio, S.; Khrennikov, A.Y.; Shelkovich, V.M. Theory of p-Adic Distributions: Linear and Nonlinear Models; London Mathematical Society Lecture Note Series, 370; Cambridge University Press: Cambridge, UK, 2010.

62. Taibleson, M.H. Fourier Analysis on Local Fields; Princeton University Press: Princeton, NJ, USA, 1975.

63. Halmos, P.R. Measure Theory; D. Van Nostrand Company, Inc.: New York, NY, USA, 1950.

64. Bourbaki, N. Éléments de mathématique. Fasc. XXXVI. Variétés différentielles et analytiques. Fascicule de résultats (Paragraphes 8 à 15); Actualités Scientifiques et Industrielles: Hermann, MO, USA; Paris, France, 1971; p. 99.

65. Weil, A. Basic Number Theory. Reprint of the Second (1973) Edition. Classics in Mathematics; Springer: Berlin/Heidelberg, Germany, 1995.

66. Rossmann, T. Computing topological zeta functions of groups, algebras, and modules, I. Proc. Lond. Math. Soc. 2015, 110, 1099-1134. [CrossRef]

67. Freund, P.G.O. $p$-adic Strings and their Applications. arXiv 2005, arXiv:Hep-th/0510192.

68. Gubser, S.S.; Jepsen, C.; Trundy, B. Spin in $p$-adic AdS/CFT. J. Phys. A 2019, 52, 144004. [CrossRef]

69. Hörmander, L. The Analysis of Linear Partial Differential Operators. I: Distribution Theory and Fourier Analysis; Springer: Berlin/Heidelberg, Germany, 2003. 